\title{
Nonlinear dynamics of radiative condensations in optically thin plasmas
}

\author{
Baruch Meerson \\ Racah Institute of Physics, Hebrew University of Jerusalem, Jerusalem 91904, Israel
}

\begin{abstract}
Plasmas that cool by radiation may become unstable to the formation of localized regions of lower temperature and increased density. Such radiative condensations are common in astrophysical and laboratory plasmas that are optically thin. Some perspective of the rapidly developing theoretical understanding of the dynamics of radiative condensations can be given in the framework of simple models. Radiative condensations are closely related to the condensation mode of the thermal instability, first studied by G. B. Field. Progress in the analysis of the nonlinear stage of this instability, achieved recently, employs the hierarchy of time and length scales of the problem. Sets of reduced equations, which describe the nonlinear dynamics of the radiative condensation, have been developed separately in the long-, intermediate-, and short-wavelength limits. Being quite different, all these reduced models predict radiation-driven segregation of the unstable plasma into two different states ("phases") on an intermediate time scale. Subsequent long-time evolution of radiative condensation may strongly depend on the type of boundary condition for the plasma. Radiative condensations usually disappear on a longer (thermal conduction related) time scale, if an inflow/outflow of the plasma is allowed. In this case, regions occupied by one of the "phases" expand until they occupy the whole plasma. On the contrary, in confined plasmas (no inflow/outflow of the plasma is allowed), radiative condensations can persist "forever." Similarities have been explored between radiative condensations and a number of instabilities of growth, such as the Darrieus-Landau instability and Ostwald ripening. A new type of shock wave with a nonmonotonic pressure profile, resulting from radiative condensation dynamics, is described. The role of magnetic fields in radiative condensations is briefly discussed.
\end{abstract}

\section{CONTENTS}

I. Introduction

II. Governing Equations, Equilibria, and Instability

III. Reduced Equations in Planar Geometry

A. Intermediate- and short-wavelength limits

B. Long-wavelength limit

IV. Isobaric Condensations

A. Unlimited instability

B. Bistability and triumph of uniformity

V. Bistability in Confined Plasmas: Triumph of Segregation

A. Radiative stage

B. Conductive stage

C. Numerical simulations

D. Intermediate-wavelength limit: summary and discussion of the planar case

VI. Radiative Condensations in the Long-Wavelength Limit

A. Unlimited instability and "anomalous" gas dynamics

B. Normal-anomalous gasdynamics and bistability

C. Numerical simulations

D. Long-wavelength limit: summary and discussion of the planar case

VII. Radiative Condensations in Two and Three

Dimensions

A. Reduced equations

B. Large-scale equilibria

C. Higher-dimensional conductive relaxation and "super-reduced" equations

D. Higher-dimensional conductive relaxation in confined plasmas

1. Linear stability of a planar interface

2. Shape stability of a perfect drop (bubble)

3. Radial stability of a perfect drop

4. Nonlocal interaction between drops: Ostwald ripening
5. Many drops' statistics and universal scalings

E. Numerical simulations

F. Transverse instability of evaporation fronts

VIII. Radiative Condensations and Magnetic Fields

A. Development of condensations across the magnetic field

B. Multifaceted asymmetric radiation from the edge of tokamak plasmas (MARFE): simple dynamic model

IX. Concluding Remarks

References

\section{INTRODUCTION}

Radiative condensations in optically thin plasmas, which are subject to some heating and cool radiatively, have attracted considerable attention since the pioneering works by Parker (1953) and Field (1965). The main motivation behind the studies of radiative condensation has been to explain the formation of dense and cool localized structures in astrophysical and laboratory plasmas, when their masses are less than those required for gravitational contraction. Indeed, the gravitational energy of solar prominences (Tandberg-Hanssen, 1974; Priest, 1989; Ruždjak and Tandberg-Hanssen, 1989) and of many types of interstellar clouds (Spitzer, 1978; Kaplan and Pikel'ner, 1979; Hollenbach and Thronson, 1987; Scheffler and Elsässer, 1987; Tenorio-Tagle et al., 1989; Burton et al., 1992) is relatively small, so that radiative condensation seems to be the only possible mechanism by which such localized dense objects can be formed from an initially homogeneous plasma. Furthermore, such laboratory plasma phenomena as radiating $Z$ pinches (Braginskii, 1957; Pease, 1957; Lawson, 1959; 242 Neudachin and Sasorov, 1991) and the so-called 
MARFEs (after the names of their discoverers, MARmar and WolFE; another version: MultifAceted Radiation From the Edge) in tokamaks (Terry et al., 1981; Alladro et al., 1982; Baker et al., 1982; Lipshultz et al., 1984; Boody et al., 1985; Lipshultz, 1987; Sergienko et al., 1993) are the best-known examples of radiative condensation in hot laboratory plasmas, while a number of radiation-related contraction phenomena are known in low-temperature plasma discharges (Nedospasov and Khait, 1979). More material in a certain region of a plasma usually implies less material in the surroundings; therefore "bubbles" and "voids," frequently discussed in different astrophysical contexts, are often intrinsically related to radiative condensation phenomena, too.

This review deals with theoretical aspects of radiative condensations. The reader is advised to consult the references given above on observational/experimental aspects of solar prominences, interstellar clouds, and MARFEs.

The simplest theoretical approach to studying radiative condensation processes employs single-component magnetogasdynamics (or even gasdynamics), with a proper account of radiative losses in the thermal balance equation. The most often studied regime is that of an optically thin plasma. In this regime, quanta, emitted in the plasma by all types of radiation processes, that contribute to the cooling leave the plasma region without reabsorption. Therefore one can account for the radiative losses by a properly constructed loss term (see Sec. II). Usually, one starts with an equilibrium (or, less often, with either a steady or an unsteady flow) and performs a linear stability analysis. If it exists, radiative condensation formation manifests itself in this approach as a so-called condensation, or radiative condensation, instability, that is, instability with respect to small density perturbations. This is an important particular case of the more general thermal instability of radiatively cooling plasmas (Parker, 1953; Kadomtsev, 1963; Field, 1965).

The basic physics of the initial growth of radiative condensation is elementary and can be conveniently explained in the following simple setting. Assume that a homogeneous optically thin plasma is heated externally, and the heating rate is uniform and constant in time. Let the plasma be in thermal equilibrium; that is, the rate of heating is balanced by the rate of radiative cooling. Now suppose that there is a local increase in the plasma density. Since the radiative cooling rate grows with the plasma density, the temperature in this region starts to fall. In order to maintain constant pressure (which is a necessity if the heating/cooling processes are slow on the acoustic time scale), plasma inflow starts, further increasing the density, and so on. If the perturbation scale is too small, the perturbation is erased by thermal conduction. Otherwise, the instability continues, and the role of theory is to predict the final state of the system and the dynamics of approaching it.

It is clear that this simplified picture can be inaccurate, or even misleading, if any of the assumptions we have made do not hold. Obviously, the constant pressure regime will not hold if the heating/cooling processes are fast on the acoustic time scale (Zel'dovich and Novikov, 1983), and we shall discuss this limit in the following. Moreover, if there are other forces, such as those due to a magnetic field (with or without shear) and/or to gravity in addition to the pressure gradient, the equilibrium can become quite complex, and even more so the stability analysis (see, e.g., Field, 1965; Defouw, 1970a; Heyvaerts, 1974; Hildner, 1974; Chiuderi and Van Hoven, 1979; Drake et al., 1988; Van der Linden et al., 1991). Additional complicating factors can be steady (Field, 1965; Schwarz et al., 1972) and unsteady (Balbus, 1988) plasma flows, more complicated ionizationrecombination dynamics (Defouw, 1970b; Goldsmith, 1970), etc. Furthermore, overly dense plasmas cease to be optically thin, so that a radiation transfer equation must be invoked. On the contrary, in overly diluted plasmas, the fluid description can break down, and a kinetic description is necessary, which makes the problem much more difficult. Because of all these complications, even the first two steps in the study of radiative condensations, equilibrium and linear stability analyses, sometimes become quite a difficult task; therefore the literature on the linear theory of radiative condensation instability in different environments is quite extensive.

Sometimes, one has a strong thermal nonequilibrium as the initial condition for radiative condensation. An important example is provided by solar prominences (Tandberg-Hanssen, 1974; Priest, 1989; Ruždjak and Tandberg-Hanssen, 1989), which are believed to develop in response to a strong, sudden perturbation: depression in the heating at the apex of a coronal magnetic loop, increased heating of the loop's footpoints, loop expansion as the result of the shearing of the footpoints, etc. (Pilkel'ner, 1971; Sasorov, 1975; Engvold and Jensen, 1977; Priest and Smith, 1979; Ribes and Unno, 1980; Poland and Mariska, 1986; Mok et al., 1990; Antiochos and Klimchuk, 1991; Choe and Lee, 1992; Drake et al., 1993). [The paper of Choe and Lee (1992) includes an extensive review on prominence formation modeling.]

Having found an instability (or, alternatively, starting from a strong nonequilibrium), one is generally interested in the subsequent dynamics. As the deviations of the physical quantities (temperature, density, etc.) from their initial values increase, we enter the nonlinear regime. The nonlinear dynamics of the radiative condensation in various contexts were addressed numerically (see Raju, 1968; Goldsmith, 1970; Schwarz et al., 1972; Oran et al., 1982; Poland and Mariska, 1986; Klimchuk et al., 1987; Mok et al., 1990; McCarthy and Drake, 1991; and Antiochos and Klimchuk, 1991 for one-dimensional simulations; and Hildner, 1974; Karpen et al., 1988, 1989; Sparks et al., 1990; Choe and Lee, 1992; and Drake et al., 1993 for two-dimensional simulations). As the full magnetogasdynamic problem with the account of thermal processes is quite difficult (especially in two and three dimensions), the basic physics of radiative condensation dynamics has remained poorly understood until quite recently. 
A complementary approach to the nonlinear theory of radiative condensation has been developing since the end of the 1980s, though its many attributes were known earlier. This approach deals with the simplest possible systems exhibiting radiative segregation, and is aimed, first of all, at understanding the basic physics behind condensation formation and evolution. In this sense its spirit is close to that of the theory of self-organization and pattern formation in a more general context (Haken, 1978, 1987; Manneville, 1990; Mikhailov, 1990; Cross and Hohenberg, 1993), where the simplest possible models, exhibiting a desirable property, are studied. The difference is that the present approach is based on a consistent reduction of the original nonlinear fluid dynamic problem, rather than on constructing convenient model equations.

In this approach, one usually starts with the simplest equilibria, for which the linear stability analysis is straightforward and predicts radiative condensation instability. Then a reduced nonlinear model is developed, which would follow the instability into the nonlinear regime. In doing so, one has to simplify the nonlinear governing equations. It is the linear theory that often gives hints on possible reductions of the problem. Indeed, the linear theory already includes the characteristic time and length scales of the problem. Reduction of the problem usually employs strong inequalities between these time or length scales. A number of reduced sets of nonlinear equations can thus be developed for different limiting cases, and they can be investigated analytically and numerically. It is important that, once the reduced models are developed, they can be employed for a variety of initial conditions, and not just for a small perturbation around thermal equilibrium. It appears that the reduced models, though they take into account only the most essential factors, are quite meaningful. While achieving a basic understanding of the physics of radiative condensation within the reduced models, one should be able to extend them by taking into account additional physical factors.

In this review a perspective of this rapidly developing complementary approach is presented. I will be trying to present the material in sufficient detail and to make this text essentially self-contained and intelligible for an advanced graduate student. For the convenience of the reader, here is a detailed plan of presentation.

Section II contains the governing fluid equations describing the basic radiative condensation processes in an unmagnetized plasma. In addition, possible spatially uniform equilibria are considered; the classical linear theory of the radiative condensation instability, developed by Field (1965), is briefly reviewed; and the characteristic time and length scales of the problem are introduced.

In Sec. III two alternative sets of reduced equations are presented, corresponding to the intermediate- and long-wavelength limits of the radiative condensation process in planar geometry. The role of boundary conditions ("open" systems versus "closed" systems) is also considered.
The intermediate-wavelength limit is reviewed in some detail in planar geometry in Secs. IV and V. In Sec. IV we start with isobaric condensations (for which the pressure is constant both in space and in time) and briefly review a number of model problems of radiative collapse or rarefaction of an "unlimitedly unstable" plasma with thermal conduction neglected. In the more practical case of bistability, radiative segregation into two phases develops on the radiative cooling time scale, and then thermal conduction starts to act. We consider possible large-scale spatially nonuniform equilibria of the bistable plasma, while taking account of thermal conduction, and show that such equilibria (corresponding to the so-called area rule) are very unlikely in "natural" isobaric systems. Instead, the regions occupied by one of the phases start expanding until all of the plasma is occupied by this phase. The expansion fronts are closely related to the condensation and evaporation fronts, first predicted by Zel'dovich and Pikel'ner (1969) and by Penston and Brown (1970) and considered in many subsequent works.

In the opposite regime of confined plasmas (Sec. V), isobaricity does not hold. Instead, under certain conditions, the uniform plasma pressure (being now a function of time) proves to approach the special value for which the area rule holds, so that a long-lived large-scale segregation equilibrium (coexistence of the two phases) is achieved. We shall interpret the pattern-forming properties of confined plasmas in terms of a nonlocal constraint (mass conservation) imposed on the system.

Section VI is devoted to the long-wavelength limit of radiative condensation dynamics in planar geometry. Here we also start with idealized problems of radiative collapse or rarefaction of an "unlimitedly unstable" plasma. Then we proceed to the more realistic case of a saturated radiative condensation and consider possible segregated equilibria, which can set in at the nonlinear stage of the instability. In this limit, the equilibria represent so-called contact discontinuities. We show that, in an open system, one of the phases starts expanding until all of the plasma becomes spatially uniform. However, this time the expansion fronts represent novel shock waves. These shock waves are quite different from those described by "conventional" gasdynamics: for example, they have a nonmonotonic pressure profile. Properties of these shocks and their role in the dynamics of radiative condensations are discussed. In the confined plasma, radiative segregation has been shown to persist "forever" as shock waves become impossible and are replaced by contact discontinuities.

In Sec. VII we extend the treatment of the intermediate-wavelength limit to two- and threedimensional systems. We discuss here both the reduced equations and the so-called super-reduced equations describing the thermal-conduction-controlled later-stage dynamics of radiatively segregated plasmas. We first concentrate on confined plasmas and delineate possible large-scale spatially nonuniform equilibria (condensation patterns). Then we review the analytical and numerical studies of (linear and nonlinear) stability of 
these patterns and of the background-mediated competition between them. Then we proceed to isobaric plasmas and review the recently discovered instability of evaporation fronts with respect to transverse perturbations, and its possible consequences in the dynamics of radiative condensations.

In Sec. VIII we briefly review two examples of radiative condensations in cases when the magnetic field plays a more active role in the dynamics. The first corresponds to plasma motions normal to a straight shearfree magnetic field. The second concerns a recent attempt to better understand the basic physics behind the formation of MARFEs in the tokamak plasma edge.

Finally, Sec. IX contains a brief summary and formulates some open problems.

\section{GOVERNING EQUATIONS, EQUILIBRIA, AND INSTABILITY}

We start with simple fluid equations (Landau and Lifshitz, 1987; Field, 1965) describing the dynamics of an unmagnetized, optically thin ideal plasma of mass density $\rho$, temperature $T$, and velocity $\mathbf{v}$, which is heated externally and cooled radiatively:

$\frac{d \rho}{d t}+\rho \nabla \cdot \mathbf{v}=0$

$\rho \frac{d \mathbf{v}}{d t}=-\nabla p+\eta \nabla^{2} \mathbf{v}+\left(\zeta+\frac{\eta}{3}\right) \nabla(\nabla \cdot \mathbf{v})$,

$\frac{1}{\gamma-1} \frac{d p}{d t}+\frac{\gamma}{\gamma-1} p \nabla \cdot \mathbf{v}+\rho \mathscr{L}(\rho, T)-\nabla \cdot(K \nabla T)=0$,

$p=\frac{R}{\mu} \rho T$,

where $d / d t=\partial / \partial t+\mathbf{v} \cdot \nabla$ is the total time derivative; $\eta$ and $\zeta$ are the viscosity coefficients; $\mathscr{L}$ is the heatingcooling function (Field, 1965), that is, the difference between the rate of radiative cooling and the rate of heating per unit mass; $K=K(T)$ is the (isotropic) thermal conductivity; $\gamma$ is the specific-heat ratio; $\mu$ is the effective molar mass of the plasma; and $R$ is the gas constant.

The form of the function $\mathscr{L}(\rho, T)$, entering the thermal balance equation, is determined by the specific mechanisms of heating and radiative cooling and varies from one application to another. The radiative cooling part of this function is conventionally written as $\rho F(T)$, where the density dependence results from the binary nature of all types of radiative collisions [notice that the heating-cooling term in Eq. (3) contains one more $\rho$ factor], while the temperature function $F(T)$ depends on the plasma composition and is determined by many radiation processes. The function $F(T)$ has been calculated in many works [see, e.g., Dalgarno and McCray (1972) and Kaplan and Pikel'ner (1979) for the interstellar medium; Lepp et al. (1985) for the quasar gas; Rosner et al. (1978) for the solar corona; and Stringer (1985) for the tokamak edge plasma]. In all these cases, this function falls abruptly when the plasma temperature becomes less than about ten thousand degrees. For temperatures larger than about $10^{7}$ degrees, the dominant mechanism of radiation is free-free radiation (also called deceleration radiation or bremsstrahlung), so that the function $F(T)$ grows like $T^{1 / 2}$. For intermediate temperatures, $F(T)$ behaves nonmonotonically. In particular, there is a large temperature interval, when it is falling with $T$ (usually for temperatures between a few hundred thousand and about $10^{7}$ degrees), which can give rise to the most primitive isochoric mode of thermal instability (Parker, 1953).

Let us return to the Navier-Stokes equation (2). All forces except the pressure gradient and small viscous friction are disregarded. This means, in particular, that we assume the plasma to be isotropic. Alternatively, we can employ the one-dimensional version of Eqs. (1)-(4), if we assume that there is a strong magnetic field, which completely suppresses both transverse plasma motions and transverse heat conduction.

The simplest possible equilibria of the system (1)-(4) are spatially uniform: $\rho=\rho_{0}=$ const, $T=T_{0}=$ const, $\mathbf{v}=0$, and $\mathscr{L}\left(\rho_{0}, T_{0}\right)=0$. In the plane of variables $\left(\rho_{0}, T_{0}\right)$, these equilibria represent curves on which the heating is exactly balanced by the radiative cooling, while the heat conduction is naturally ineffective, or "switched off." Sometimes the equation $\mathscr{C}=0$ may have no "nontrivial" solutions, so that no uniform equilibrium is possible. (The simplest example for this is a nonzero radiative cooling with no heating. In this case the plasma will cool off until its temperature falls to the "cutoff" temperature of a few thousand degrees, when the radiative cooling vanishes.) Additional constraints on the spatially uniform equilibria can be imposed by the boundary conditions of the problem. For example, if the plasma is in contact with a boundary, which has a prescribed constant temperature, this value of the temperature is the only possible "candidate" for uniform $T_{0}$.

If these idealized, spatially uniform equilibria do exist, we can study their stability with respect to small perturbations. This problem was solved by Field (1965). He showed that it is the behavior of the heating-cooling function $\mathscr{B}(\rho, T)$ in the vicinity of the equilibrium values $\rho_{0}$ and $T_{0}$, the value of thermal conductivity $K\left(T_{0}\right)$, and the perturbation wave number $k=\left(k_{x}^{2}+k_{y}^{2}+k_{z}^{2}\right)^{1 / 2}$ that control thermal stability and, in the case of instability, its type (isochoric, isobaric, or isentropic) and growth rate. We shall briefly describe the results of the linear theory by Field, first introducing the characteristic time and spatial scales of the problem. Assume that the plasma viscosity is zero (we shall return to a nonzero viscosity in Sec. VI). Let $\lambda=2 \pi / k$ be the perturbation wavelength. Then $\tau_{a}=\lambda / c_{s}$ is the characteristic acoustic time scale, where $c_{s}$ is the acoustic speed. Now, introduce the thermal conduction time scale $\tau_{c}=\lambda^{2} / \kappa$, where $\kappa=K / \rho_{0}$ is the thermal diffusivity. Finally, introduce the characteristic radiative cooling time scale $\tau_{r}$, which is independent of the perturbation wavelength. Correspondingly, we can introduce the characteristic length scales similar to those defined by Field (1965). The acoustic length $\lambda_{a}$ is defined as $c_{s} \tau_{r}$, while the con- 
ductive length $\lambda_{c}$ is defined as $\left(\kappa \tau_{r}\right)^{1 / 2}$. Normally, the acoustic length is much longer than the conductive one, $\lambda_{a} \gg \lambda_{c}$. We can say, therefore, that perturbations with $\lambda \gg \lambda_{a}$ correspond to the long-wavelength limit; those with $\lambda_{c} \ll \lambda \ll \lambda_{a} \quad\left(\tau_{c} \gg \tau_{r} \gg \tau_{a}\right)$, to the intermediatewavelength limit; and those with $\lambda \leqslant \lambda_{c}$, to the shortwavelength limit. This classification is of great importance to the following discussion.

Linearizing Eqs. (1)-(4) (with $\eta=\zeta=0$ ) around a uniform equilibrium and looking for solutions proportional to $\exp (n t+i \mathbf{k} \cdot \mathbf{r})$, Field (1965) arrived at a cubic dispersion relation for the growth (damping) rate $n$, which describes three coupled modes. He analyzed this dispersion relation numerically and analytically and found the instability conditions for all modes. We shall be interested in the radiative condensation instability mode only and formulate the results of the linear theory separately in two different limits.

In the combined intermediate- and short-wavelength limits, the results are the following. If the uniform plasma equilibrium is isochorically stable,

$$
\left(\frac{\partial \mathscr{B}}{\partial T}\right)_{\rho}>0
$$

it is the isobaric instability criterion,

$$
\left(\frac{\partial \mathscr{L}}{\partial T}\right)_{p}=\left(\frac{\partial \mathscr{B}}{\partial T}\right)_{\rho}-\frac{\rho}{T}\left(\frac{\partial \mathscr{D}}{\partial \rho}\right)_{T}<0
$$

for $\rho=\rho_{0}$ and $T=T_{0}$, that represents the necessary condition for the radiative condensation instability (Field, 1965). The growth rate is equal to

$$
n_{i s}=\frac{(\gamma-1) \mu}{\gamma R}\left[-\left(\frac{\partial \mathscr{C}}{\partial T}-\frac{\rho_{0}}{T_{0}} \frac{\partial \mathscr{B}}{\partial \rho}\right)-\kappa_{0} k^{2}\right],
$$

evaluated at $T=T_{0}$ and $\rho=\rho_{0}$. Here $\kappa_{0}=K\left(T_{0}\right) / \rho_{0}$ is the unperturbed thermal diffusivity. Equation (7) follows from the cubic dispersion relation by Field in the intermediate- and short-wavelength limits, $k \gg \lambda_{a}^{-1}$. Since the radiative loss rate in a plasma grows with $\rho$, the second term in Eq. (5) is destabilizing. As seen from Eq. (7), in the intermediate-wavelength limit, the growth rate is almost independent of the wave number (plateau regime). In the short-wavelength range, thermal conduction starts to act, and it always has a stabilizing effect, erasing perturbations with wavelengths shorter than some threshold wavelength (which typically is of the order of the conductive length).

In the long-wavelength limit, $k \ll \lambda_{a}^{-1}$, the three above-mentioned modes are the "isochoric" mode (unstable or stable), the growth (or damping) rate of which is generally nonzero, while the real part of the frequency is zero as $k \rightarrow 0$, and two "acoustic" modes, whose frequencies both tend to zero as $k \rightarrow 0$. When the isochoric stability criterion (5) is not satisfied, the two remaining modes represent propagating acoustic waves modified by the heating-cooling effects. In the opposite case, $\left(\partial \mathscr{C} / \partial T_{0}\right)_{\rho}>0$, the "acoustic" modes become nonpropagating (one of the modes purely growing; the other, damped), if the isobaric instability criterion (6) is satis-

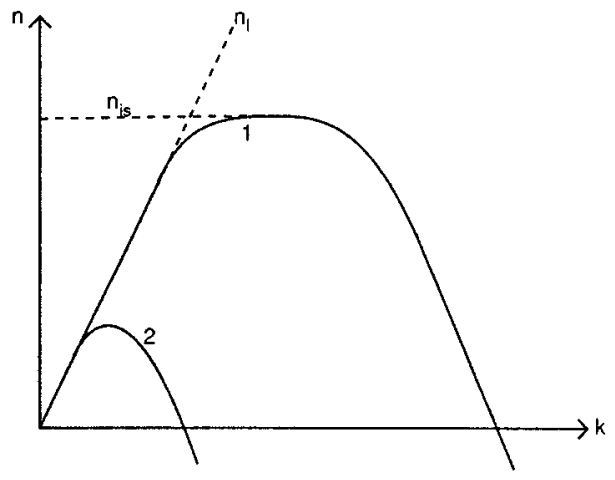

FIG. 1. Linear growth rate of the radiative condensation instability vs the perturbation wave number $k$, and its asymptotics [after Field (1965) and Meerson, Steele et al. (1993)].

fied. The growth rate of this long-wavelength radiative condensation instability can be easily found from the general dispersion relation by Field (1965):

$$
n_{l}= \pm k c_{i}\left\{-\left[\left(\frac{\partial \mathscr{B}}{\partial T_{0}}\right)_{\rho}-\frac{\rho_{0}}{T_{0}}\left(\frac{\partial \mathscr{B}}{\partial \rho_{0}}\right)_{T}\right] /\left(\frac{\partial \mathscr{L}}{\partial T_{0}}\right)\right\}_{\rho}^{1 / 2}
$$

(Meerson, Steele et al., 1993) where $c_{i}^{2}=R T_{0} / \mu$. Again, the real part of the mode frequency is zero. Notice that despite the isobaric criterion for instability, the mode itself is not isobaric in this limit, the pressure varying both in space and in time.

The linear increase of the growth rate $n_{l}$ with the wave number $k$, predicted by Eq. (8), terminates at $k \sim \lambda_{a}^{-1}$, i.e., outside the long-wavelength limit. If $k \gg \lambda_{a}^{-1}$, we arrive at the intermediate- and shortwavelength limits with the growth rate (7). Figure 1 (curve 1 and its two asymptotics) shows schematically the dependence of the linear growth rate on the wave number $k$ for the case of $\lambda_{c} \ll \lambda_{a}$, when all three ranges-the long-, intermediate-, and short-wavelength ones-are well pronounced. Shown is the plot of the growth rate $n=n(k)$ and its asymptotics $n_{i s}$ and $n_{l}$ from Eqs. (7) and (8), respectively. One can see that the maximum growth rate is achieved in the intermediatewavelength limit. Roughly speaking, the typical growth time in this limit is of the order of the radiative cooling time $\tau_{r}$. Curve 2 in Fig. 1 corresponds to the case when $\lambda_{a} \ll \lambda_{c}$ (Meerson, Steele et al., 1993).

\section{REDUCED EQUATIONS IN PLANAR GEOMETRY}

Having found an instability with respect to small perturbations, one should ask, what determines the further evolution of the unstable perturbations. Will the system finally reach a stable equilibrium, develop a regular flow, or reach a more complicated (even turbulent) state? Is the instability weak or strong (in other words, will it saturate at a low or a high level of perturbation)? If a stable equilibrium is finally reached, will it be spatially uniform or nonuniform (segregated)? To address these questions, one clearly needs to go beyond the linear 
theory. The governing set of nonlinear equations (1)-(4) cannot be solved analytically. As to numerical solutions, they usually address specific problems and particular choices of parameters and therefore may not give an overall picture. To try and achieve such a picture, we should simplify the governing equations. The presence of different characteristic length and time scales in the problem makes it possible to reduce the governing set of equations separately in the combined intermediate- and short-wavelength limits and in the long-wavelength limit.

The momentum equation (2) and thermal balance equation (3) describe relaxation of the system to pressure equilibrium and thermal equilibrium, respectively. In the intermediate- and short-wavelength limits, it is pressure balance that sets in first, on the acoustic time scale. Then a slower evolution proceeds on the radiative cooling time scale and, later, on the thermal conduction time scale. Therefore if we are interested in this slower evolution, we can assume that pressure balance has already set in (Sasorov, 1988; Meerson, 1989). This approach implies that we are not interested in the shorttime dynamics developing on the acoustic time scale.

Alternatively, in the long-wavelength limit, the acoustic time scale becomes relatively long, and it is thermal equilibrium that sets in first. Therefore, to investigate the later stages of the dynamics, which now develop on the acoustic time scale, we can assume that thermal balance has already set in. In this case, the radiative cooling time is the shortest time of the problem, and we are not interested in what is happening on this shortest time scale. Obviously, in doing so, we deliberately "overlook" radiative cooling dynamics [in particular, the development of the most primitive isochoric instability; see Parker (1953) and Field (1965)]. More precisely, we assume that, on the shortest, radiative cooling, time scale, the system "jumps" to an isochorically stable state, and it is the subsequent slower dynamics that we are interested in (Meerson and Sasorov, 1987).

\section{A. Intermediate- and short-wavelength limits}

Let us consider the intermediate- and shortwavelength limits in planar geometry. As mentioned above, pressure balance sets in rapidly, so that we can replace, in the zero approximation, the complete momentum equation (2) by the simple relation

$$
\frac{\partial p}{\partial x}=0
$$

that is, the pressure becomes uniform in space. The rest of the equations take the following form:

$$
\begin{aligned}
& \frac{d \rho}{d t}+\rho \frac{\partial v}{\partial x}=0 \\
& \frac{1}{\gamma-1} \frac{d p}{d t}+\frac{\gamma}{\gamma-1} p \frac{\partial v}{\partial x}+\rho \mathscr{L}(\rho, T)-\frac{\partial}{\partial x}\left(K \frac{\partial T}{\partial x}\right)=0
\end{aligned}
$$

$$
p=\frac{R}{\mu} \rho T,
$$

where $d / d t=\partial / \partial t+v \partial / \partial x$, except in Eq. (11), where $d p / d t=\partial p / \partial t$ by Eq. (9).

One can easily check that the linearization of the reduced equations (9)-(12) around a uniform equilibrium predicts the correct growth rate (7).

It is convenient to transfer to scaled variables. First, we assume that the temperature dependence of the thermal conductivity is powerlike: $K(T)=K_{0} T^{\alpha}$, so that the cases of electron-dominated $(\alpha=5 / 2)$ and neutraldominated $(\alpha=1 / 2)$ thermal conductivity can be accounted for properly. Instead of the plasma density, we introduce the specific volume, $u(x, t)=\rho^{-1}(x, t)$, and eliminate the temperature $T(x, t)$, using the equation of state (12). Now the heating-cooling function $\mathscr{C}(\rho, T)=\mathscr{C}\left[u^{-1},(\mu / R) p u\right]$ depends on $u$ and $p$. Introducing scaled variables $\hat{u}=u / u_{0}$ and $\hat{p}=p / p_{0}$, we define the dimensionless heating-cooling function $\lambda_{E}(\hat{u}, \hat{p})$ :

$$
\frac{\gamma-1}{\gamma p u} \mathscr{L}\left(u^{-1}, \frac{\mu}{R} p u\right)=\mathscr{C}_{0} \lambda_{E}(\hat{u}, \hat{p}),
$$

where the parameters $u_{0}$ and $p_{0}$ and the coefficient $\mathscr{B}_{0}$ are chosen in such a way that the function $\lambda_{E}(\hat{u}, \hat{p})$, evaluated, for example, at $\hat{u}=1$ and $\hat{p}=1$, is equal to unity. In this notation, the conductive Field length $\delta_{F}$ (Begelman and McKee, 1990) is the following:

$$
\delta_{F}^{2}=\left(\frac{\mu}{R}\right)^{1+\alpha} \frac{(\gamma-1) K_{0} p_{0}^{\alpha} u_{0}^{1+\alpha}}{\gamma \mathscr{L}_{0}} .
$$

Now we can introduce the remaining scaled variables- $\hat{x}=x / \delta_{F}, \hat{t}=\mathscr{L}_{0} t$, and $\hat{v}=v /\left(\delta_{F} \mathscr{C}_{0}\right)$-and rewrite Eqs. (10) and (11) in the following form:

$$
\begin{aligned}
& \frac{\partial u}{\partial t}=u \frac{\partial v}{\partial x}-v \frac{\partial u}{\partial x}, \\
& \frac{\dot{p}}{\gamma p}+\frac{\partial v}{\partial x}+\lambda_{E}(u, p)-p^{\alpha} \frac{\partial}{\partial x}\left(u^{\alpha} \frac{\partial u}{\partial x}\right)=0,
\end{aligned}
$$

where $\dot{p} \equiv d p(t) / d t$ and the "hats" are omitted. The two equations, (15) and (16), include three variables, $u(x, t), v(x, t)$, and $p(t)$; therefore some additional information must be used. Such information is normally provided by the boundary conditions. There are many possible types of boundary conditions, depending on the specific problem. We shall consider here two cases, which, in some sense, complement each other.

The first is the case of isobaricity, $p=$ const. It corresponds to a prescribed, constant value of the pressure, necessarily the same at both boundaries. This condition models the situation when the plasma region in question is surrounded by a large amount of ambient plasma (plasma reservoir). In this case Eq. (16) reduces to the following relation:

$$
\frac{\partial u}{\partial x}+\lambda_{E}(u, 1)-\frac{\partial}{\partial x}\left(u^{\alpha} \frac{\partial u}{\partial x}\right)=0,
$$

where we put $p=1$. Notice that in the isobaric regime we can replace the (scaled) specific volume $u$ by the (scaled) plasma temperature $T$. 
Equations (15) and (17) (Meerson, 1989) are much simpler than the original set of Eqs. (1)-(4), and they represent the planar isobaric version of the reduced equations, valid in the intermediate- and shortwavelength limits. They should be supplemented by two boundary conditions for the specific volume (or temperature) and by one boundary condition for the velocity.

The second case corresponds to a plasma volume, confined by external forces, so that no plasma can enter or leave the system. As an astrophysical example of such a confinement, one can consider interstellar gas in the gravitational field of a galactic disk. Of course, a fully consistent treatment of this problem would require an explicit account of gravitation in the equation of motion. Instead, we effectively replace a smooth gravitational potential well by a one-dimensional "box" of length $L$, so that the plasma inside the box does not feel gravity until it reaches the "walls." Technically, we assume that the plasma velocity vanishes at both boundaries. From this immediately follows mass conservation:

$$
\int_{0}^{L} \frac{d x}{u(x, t)}=M=\text { const. }
$$

It is natural to prescribe the no-flux boundary conditions for the density (or, equivalently, temperature), which means $\partial u / \partial x=0$ at $x=0$ and $x=L$. Then, integrating Eq. (16) over $x$ from 0 to $L$, we arrive at the following nonlocal relation:

$$
\frac{\dot{p}}{\gamma p}=-\frac{1}{L} \int_{0}^{L} d x \lambda_{E}(u, p),
$$

which represents an evolution equation for the pressure and makes the set of reduced equations (15), (16), and (19) closed (Aranson et al., 1993). Equation (19) is very similar to the "global" pressure equation used by Begelman and McKee (1990) for a qualitative analysis of the global effects of thermal conduction on "two-phase" media. Essentially, Eq. (19) corresponds to a particular limit of Begelman and McKee's analysis. They call this case isochoric in the sense that "the mass is effectively contained within a fixed volume" (p. 378). We call the same case confined plasma, or closed system.

Notice that the isobaric reduced equations (15) and (17) can be formally obtained from the confined plasma reduced equations (15), (16), and (19), if we formally put $L=\infty$ in the global pressure equation (19) and return from the specific volume to the temperature variable.

In both cases, the reduced equations look simpler in Lagrangian mass coordinates. Let us start with the confined plasma equations. Introduce the (scaled) Lagrangian mass coordinate

$$
m=\int_{0}^{x} \frac{d x}{u(x, t)} .
$$

Then, transferring from variables $x$ and $t$ to the new variables $m$ and $t$ in Eqs. (15), (16), and (19), we arrive at only two coupled equations (Aranson et al., 1993):

$$
\begin{aligned}
& \frac{\partial u}{\partial t}+\lambda_{L}(u, p)-p^{\alpha} \frac{\partial}{\partial m}\left(u^{\alpha-1} \frac{\partial u}{\partial m}\right)+\frac{u \dot{p}}{\gamma p}=0, \\
& \frac{\dot{p}}{\gamma p}=-\frac{1}{L} \int_{0}^{M} d m \lambda_{L}(u, p),
\end{aligned}
$$

where $\lambda_{L}(u, p)=u \lambda_{E}(u, p)$, with the boundary conditions $\partial u / \partial m=0$ at $m=0$ and $m=M$.

Having found $u(m, t)$ and $p(t)$ from Eqs. (21) and (22), we can easily determine the plasma velocity

$$
v(m, t)=\int_{0}^{m} \frac{\partial u}{\partial t} d m^{\prime}=\frac{\partial}{\partial t} \int_{0}^{m} u d m^{\prime} .
$$

Finally, the relationship between Eulerian and Lagrangian coordinates, necessary for a transformation to Eulerian coordinates $x$ and $t$, is given by

$$
x(m, t)=\int_{0}^{m} u\left(m^{\prime}, t\right) d m^{\prime} .
$$

The mass conservation integral (18) looks trivial in the Lagrangian coordinates: $M=$ const; that is, the Lagrangian "length" of the box is constant. On the other hand, the constancy of the "true" (that is, Eulerian) box length, $L=$ const, while trivial in the Eulerian coordinates, looks like a conservation law in the Lagrangian coordinates:

$$
\int_{0}^{M} u(m, t) d m=L=\text { const. }
$$

In the isobaric case, $p=$ const $=1$, the Lagrangian coordinates leave us with only one evolution equation:

$$
\frac{\partial u}{\partial t}+\lambda_{L}(u, 1)-\frac{\partial}{\partial m}\left(u^{\alpha-1} \frac{\partial u}{\partial m}\right)=0 .
$$

This equation was obtained by Doroshkevich and Zel'dovich (1981) in the context of a radiative cooling wave, and later by Meerson (1989) in the context of the radiative condensation instability. Equation (26) belongs to a broad class of the so-called generalized reactiondiffusion equations.

In contrast to the confined plasma regime, the mass content of any fixed Eulerian region is not preserved. Therefore, working with Eq. (26), one should remember that the Lagrangian "length" $M$ of the computation interval changes in time. Finally, when $u(m, t)$ is determined, one finds the velocity from Eq. (23) and returns to the Eulerian coordinates using Eq. (24).

\section{B. Long-wavelength limit}

Let us proceed now to the long-wavelength limit. In this limit, the local thermal equilibrium sets in rapidly, so that we can replace the complete thermal balance equation (3) by the equilibrium condition

$$
\mathscr{B}(\rho, T)=0
$$

(thermal conduction can be usually neglected for the long wavelengths). Solving the algebraic equation (27) 
for $T=T_{*}(\rho)$ and substituting it into equation of state (4), we obtain the plasma pressure

$$
p=(R / \mu) \rho T_{*}(\rho)=P(\rho),
$$

which is a function of the density only, so that the planar version of Eqs. (1) and (2) with the effective pressure $p=P(\rho)$ represents a closed set:

$$
\begin{aligned}
& \frac{d \rho}{d t}+\rho \frac{\partial v}{\partial x}=0, \\
& \rho \frac{d v}{d t}=-\frac{\partial p}{\partial x}+\eta \frac{\partial^{2} v}{\partial x^{2}} .
\end{aligned}
$$

These are the planar long-wavelength reduced equations for the radiative condensation instability of an isochorically stable plasma (Zel'dovich and Novikov, 1983; Meerson and Sasorov, 1987; Meerson, Steele et al., 1993). Pikel'ner (1967) was the first to recognize that the effective pressure $P$ of an optically thin, radiatively cooling plasma with a cosmic abundance of elements, which is heated by UV radiation and subcosmic rays, can be a nonmonotonic function of the density, which can lead to instability. Indeed, linearizing the reduced equations near the equilibrium $\rho=\rho_{0}$ and $v=0$, and looking for solutions in the form of $\exp (n t+i k x)$, we obtain (Zel'dovich and Novikov, 1983; Meerson, Steele et al., 1993)

$$
n= \pm k\left(-\frac{d P}{d \rho_{0}}+\frac{\nu^{2} k^{2}}{4}\right)^{1 / 2}-\frac{\nu k^{2}}{2}
$$

where $\nu=\eta / \rho_{0}$ is the unperturbed kinematic viscosity. It can be seen from Eq. (30) that a necessary and sufficient condition for the instability of one of the two "acoustic" modes is

$$
\frac{d P}{d \rho_{0}}<0
$$

which formally means negative gas compressibility. The viscosity reduces the growth rate, but it is unable to quench the instability. An illustrative example of the effective pressure, for which the instability can develop, is shown in Fig. 2, where density intervals $(0,1)$ and $(3, \infty)$ are stable, while interval $(1,3)$ is unstable (normalized density and pressure units are used). The linear growth rate (30) increases monotonically with $k$ and becomes saturated, approaching $-\nu^{-1} d P / d \rho_{0}$ as $k \rightarrow \infty$ (it should be remembered, however, that the longwavelength theory breaks down at $\left.k \geqslant \lambda_{a}^{-1}\right)$.

Of course, the growth rate (30), with no viscosity, exactly coincides with the long-wavelength asymptotics (8) of the "complete" linear growth rate [that is, that following from the cubic dispersion relation by Field (1965)]. To prove it, we return to the heating-cooling function $\mathscr{B}(\rho, T)$, calculate $d P / d \rho_{0}$ at $\mathscr{B}=$ const, and substitute the result in Eq. (30). We immediately arrive at Eq. (8).

In addition to the long-wavelength limit of the radiative condensation instability, Eqs. (28) and (29), with a nonmonotonic effective pressure $p=P(\rho)$, describe

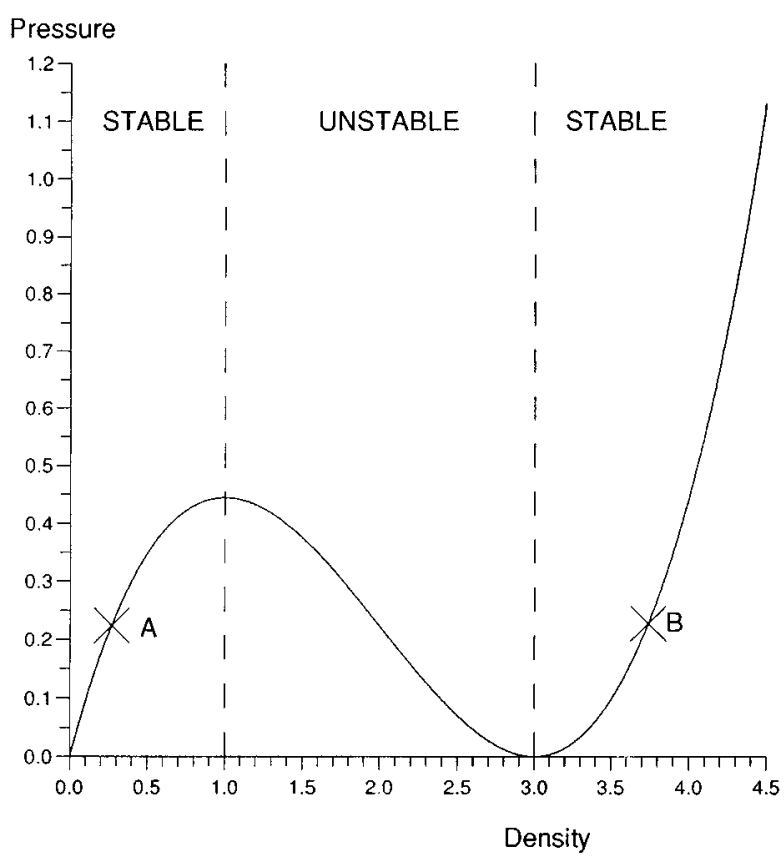

FIG. 2. Effective pressure $P(\rho)$, showing negative gas compressibility on the interval $(1,3)$. Stable points A and B have the same pressure but different density.

other physical systems. The best known of them is the van der Waals model for real gases, for which the equation of state is

$$
p(\rho, T)=\frac{8 T \rho}{3-\rho}-3 \rho^{2}
$$

(see, e.g., Kittel and Kroemer, 1980), where all the quantities (pressure, density, and temperature) are related to their critical values. For $T<1$, the pressure is a nonmonotonic function of $\rho$, the falling part of which corresponds to the instability of the overcooled vapor. If one "quickly" prepares (for instance, in a Wilson cloud chamber) a uniform equilibrium state of such a vapor and ensures that the subsequent dynamics is isothermal (or, more generally, polytropic with certain limitations on the polytrope index), an instability similar to the long-wavelength radiative condensation instability will develop.

Therefore we have developed two sets of reduced equations, which correctly reproduce the predictions of the linear theory by Field (1965) in the long-, intermediate-, and short-wavelength limits. The two "subsets," obtained in the intermediate- and shortwavelength limits, account for different types of boundary conditions: those for an isobaric plasma, and those for a confined plasma with no heat and mass exchange through the boundaries. The reduced equations are applicable for nonlinear problems as well, unless a new time or length scale appears in the dynamics that does not satisfy the assumed hierarchy.

\section{ISOBARIC CONDENSATIONS}

We have seen that the dynamics of intermediate- and short-wavelength isobaric perturbations in a radiatively 
cooling plasma is described, in Lagrangian coordinates, by the generalized reaction-diffusion equation (26). Similar one-component reaction-diffusion equations have appeared in numerous applications in physics, chemistry, and biology, starting from the pioneering works by Fisher (1936) and Kolmogorov et al. (1937) in the context of mathematical biology. Different aspects of this equation have been discussed quite extensively (for reviews and monographs see Frank-Kamenetskii, 1969; Haken, 1978; Fife, 1979; Akhromeyeva et al., 1989; Murray, 1989; Mikhailov, 1990; and Cross and Hohenberg, 1993). Obviously, the properties of this equation are determined by the form of function $\lambda_{L}(u, 1)$ and by the parameter $\alpha$. As no analytic solution of the reactiondiffusion equation is generally available, most of the analytic work on this equation concentrated on traveling-wave solutions (Frank-Kamenetskii, 1969; Fife, 1979; Murray, 1989; Mikhailov, 1990) and localized "explosive" solutions (Akhromeyeva et al., 1989). We shall start with another, much simpler type of solution by considering the intermediate-wavelength limit. In this limit, the last term of Eq. (26) is small. Neglecting it, we arrive at a very simple equation (Meerson, 1989),

$$
\frac{\partial T}{\partial t}+\lambda(T)=0,
$$

where we have transferred to the temperature variable and put $\lambda_{L}(T, 1) \equiv \lambda(T)$. Obviously, the solution will depend on the form of the isobaric heat-loss function $\lambda(T)$ and on the initial condition. Let $\lambda(T)$ have a zero (let it be at $T=1$ ), which is unstable with respect to small perturbations. This implies that the regions where the initial condition $T(m, t=0)>1$ will heat up, while the regions where $T(m, t=0)<1$ will cool down. Since the pressure remains constant, the heated plasma will become more dilute, while the cooling plasma will become denser. The (subacoustic) velocity field (23) will immediately develop to evacuate material from the hotter region to the cooler regions. We see that the Lagrangian coordinates provide an exceptionally simple and natural framework for the description of a planar radiative condensation, including its strongly nonlinear stage. The following dynamics of the condensation are determined by the form of the isobaric heat-loss function $\lambda(T)$.

\section{A. Unlimited instability}

If $\lambda(T)$ does not have additional zeros, instability develops without limit: either $T \rightarrow 0, \rho \rightarrow \infty$ (collapse), or $T \rightarrow \infty, \rho \rightarrow 0$ (unlimited rarefaction). For some $\lambda(T)$, these singularities can develop in a finite time. For example, "explosive" rarefaction will develop if $|\lambda(T)|$ grows faster than const $\cdot T$ as $T \rightarrow \infty$. It is quite easy to obtain different analytical solutions describing this idealized unlimited instability. In some cases, it is even possible to make the transformation back to the Eulerian coordinates analytically, as in the following example of explosive rarefaction (Meerson, 1989). Let us choose the scaled isobaric heating-cooling function in the form

$$
\lambda(T)=T-T^{2} .
$$

Here we have an unstable equilibrium point $T=1$ and a stable point $T=0$. The heating rate of regions with $T(m, t=0)>1$ increases rapidly with the temperature; therefore the singularity $T \rightarrow \infty$ occurs at a point where $T(m, t=0)$ has the absolute maximum. As an initial condition, consider a small sinusoidal density perturbation (with the amplitude $\epsilon \ll 1$ ) around the unstable equilibrium. Then, using Eqs. (33), (34), and (23), we obtain simple, explicit formulas for the flow variables in the Lagrangian coordinates:

$$
\begin{aligned}
\rho(m, t)= & 1-\epsilon e^{t} \cos m, \\
T(m, t)= & \left(1-\epsilon e^{t} \cos m\right)^{-1}, \\
v(m, t)= & \frac{\epsilon e^{t} \sin m}{\left(1-\epsilon^{2} e^{2 t}\right)\left(1-\epsilon e^{t} \cos m\right)} \\
& +\frac{2 \epsilon^{2} e^{2 t}}{\left(1-\epsilon^{2} e^{2 t}\right)^{3 / 2}} \arctan \left[\left(\frac{1+\epsilon e^{t}}{1-\epsilon e^{t}}\right)^{1 / 2} \tan \frac{m}{2}\right] .
\end{aligned}
$$

The relationship between the Eulerian and Lagrangian coordinates follows from Eq. (24):

$$
x(m, t)=\frac{2}{\left(1-\epsilon^{2} e^{2 t}\right)^{1 / 2}} \arctan \left[\left(\frac{1+\epsilon e^{t}}{1-\epsilon e^{t}}\right)^{1 / 2} \tan \frac{m}{2}\right] .
$$

This simple example proves to be quite instructive, because, using Eq. (38), we can calculate explicitly the flow variables in the Eulerian coordinates:

$$
\begin{aligned}
& \rho(x, t)=\frac{1-\epsilon^{2} e^{2 t}}{1+\epsilon e^{t} \cos \left(\sqrt{1-\epsilon^{2} e^{2 t} x}\right)}, \\
& T(x, t)=\frac{1+\epsilon e^{t} \cos \left(\sqrt{1-\epsilon^{2} e^{2 t}} x\right)}{1-\epsilon^{2} e^{2 t}}, \\
& v(x, t)=\frac{\epsilon e^{t}}{\left(1-\epsilon^{2} e^{2 t}\right)^{3 / 2}} \sin \left(\sqrt{1-\epsilon^{2} e^{2 t}} x\right)+\frac{\epsilon^{2} e^{2 t} x}{1-\epsilon^{2} e^{2 t}} .
\end{aligned}
$$

It can be directly checked that these solutions satisfy the set of the "Eulerian" equations (15) and (17) with $\lambda(T)$ from Eq. (34). The character of the singularities in $T(x, t)$ and $v(x, t)$ as $t \rightarrow \ln (1 / \epsilon)$ is clearly seen from Eqs. (40) and (41).

In this and other idealized examples (see Meerson, 1989), radiative condensations and rarefactions arise in "exaggerated" forms (singularities developing on a finite time). More realistic shapes of the heating-cooling function prevent these singularities from forming. However, the singularities clearly demonstrate that the radiative condensation instability is a "strong" instability in the sense that weak nonlinearities are usually unable to arrest the condensation growth, and they even may accelerate it. [This statement also holds in the opposite, longwavelength limit (see later), where the mathematical model is quite different.] 


\section{B. Bistability and triumph of uniformity}

More realistic is the case of bistability, when the function $\lambda(T)$ has more than one stable zero. It is thought to be typical, for example, for the interstellar medium, where there can be several such zeros [that is, several possible equilibrium "phases" of the gas, when heating (usually by UV radiation and cosmic rays) is balanced by radiative cooling (Pikel'ner, 1967; Field et al., 1969; Lepp et al., 1985)]. In this case, those zeros of $\lambda(T)$ that are adjacent to the "unstable" zero at $T=1$ are stable. The overcooled regions where $T(m, t=0)<1$ will continue to cool down and finally approach the lowertemperature stable equilibrium (let it be $T_{1}$ ), while the overheated regions with $T(m, t=0)>1$ will continue to heat up and finally approach the higher-temperature stable equilibrium $T_{2}$. In other words, the plasma will segregate into a two-phase state, in which lowtemperature, high-density "condensations" (phase 1) are surrounded by high-temperature, low-density "voids" (phase 2). For $t \gg \tau_{r}$, the temperature will develop a "rectangular" profile (alternating domains of phases 1 and 2 with infinitely thin boundaries between them). The positions of the interphase boundaries in the Lagrangian coordinates coincide with the zeros of the function $T(m, t=0)-1$. The whole process of segregation of a thermally bistable gas can be easily described analytically with the aid of Eq. (33) if we choose, for example, a cubic polynomial heat-loss function with three zeros, $T_{1}, 1$, and $T_{2}$ (Meerson, 1989).

However, as large temperature gradients develop at the boundaries between the two phases, thermal conduction [the last term in Eq. (26)] becomes important. Obviously, thermal conduction broadens the interphase boundaries: the width of the transition layer becomes of the order of the conducting Field length (that is, of the order of unity in our scaled equations). Furthermore, in the general case, the interphase boundaries start to move, and traveling fronts [they are called trigger waves or switching waves in other applications; see Mikhailov (1990)] develop. These fronts resemble traveling fronts of the first-order phase transitions, such as solidification or melting fronts (Kurz and Fisher, 1992), flame propagation in combustion (Zel'dovich et al., 1985), etc. In the course of time, a single interphase boundary approaches a traveling-wave solution. Such solutions were considered, in the context of externally heated and radiatively cooled bistable plasmas, in many works, starting with Zel'dovich and Pikel'ner (1969), and Penston and Brown (1970). (In these early works the plasma motion was not accounted for properly.) An updated bibliography on planar conductive/cooling fronts can be found in the paper by Ferrara and Shchekinov (1993). In the general context of the one-component reaction-diffusion equation with bistability, the traveling-wave solutions were studied in a much larger number of works (see the books cited in the beginning of this section and the references therein).

In our notation, the results of these studies are the following (Dimits and Meerson, 1991). The direction of motion of a single traveling front is uniquely determined by the sign of the following integral:

$$
I=\int_{T_{1}}^{T_{2}} T^{\alpha-1} \lambda(T) d T \equiv \int_{T_{1}}^{T_{2}} T^{\alpha} \lambda_{E}(T, 1) d T .
$$

If $I<0$, the traveling front will move from the lowtemperature side to the high-temperature side (condensation front). If $I>0$, the traveling front moves in the opposite direction (evaporation front). In the special case of $I=0$ (we shall call it the area rule), the front is standing, rather than moving. Both the propagation speed and the form of the traveling front in a bistable medium are uniquely determined by the form of the function $\lambda(T)$ and by the value of $\alpha$. Unfortunately, no analytic solution is generally possible for an arbitrary $\lambda(T)$ and $\alpha$. A number of approximate methods for the calculation of propagation speed were developed in different limiting cases [they are reviewed by Mikhailov (1990)]. In addition, a number of useful exact analytic solutions exist for $\alpha=1$ and some specially chosen forms of $\lambda(T)$ (Frank-Kamenetskii, 1969). For example, for the cubic polynomial heat-loss function,

$$
\lambda(T)=\left(T-T_{1}\right)(T-1)\left(T-T_{2}\right),
$$

a single temperature solution has the following form:

$T(\xi)=\frac{T_{1}+T_{2}}{2}+\frac{T_{2}-T_{1}}{2} \tanh \left( \pm \frac{T_{2}-T_{1}}{2 \sqrt{2}} \xi+C\right)$,

where $\xi=m-V t, C$ is an arbitrary constant, the upper sign corresponds to the case $T(\xi) \rightarrow T_{1}$ as $\xi \rightarrow-\infty$ and $T(\xi) \rightarrow T_{2}$ as $\xi \rightarrow+\infty$, and the lower sign to the reverse. The front propagation velocity $V$ is

$$
V= \pm \sqrt{2}\left[1-\left(T_{1}+T_{2}\right) / 2\right]
$$

with the same sign rule as before. Using Eq. (44), one can easily calculate the density and velocity for this traveling-wave solution. Although these relations are obtained in Lagrangian coordinates, one can check that any quantity that is of the form of a steadily moving front in the Lagrangian coordinates is also a steadily moving front in the original, Eulerian coordinates (Dimits and Meerson, 1991).

The value of the parameter $I$ in any specific problem is determined (for given mechanisms of heating and cooling) by the plasma pressure. Therefore there exists a (very special) area-rule value of the pressure, for which a single front is standing.

Let us return to the general intermediate-wavelength initial conditions (Dimits and Meerson, 1991). We have seen that in the first, radiative, stage of the dynamics, a "rectangular" temperature (and density) profile develops. Roughly speaking, the duration of the radiative stage is of the order of the radiative cooling time (if the values of $T_{1}$ and $T_{2}$ do not introduce very large or very small parameters). During the much longer second stage (we call it conductive), each of the interphase boundaries develops a traveling front solution. These traveling fronts move almost independently from one another until two of them approach each other (that is, until the 


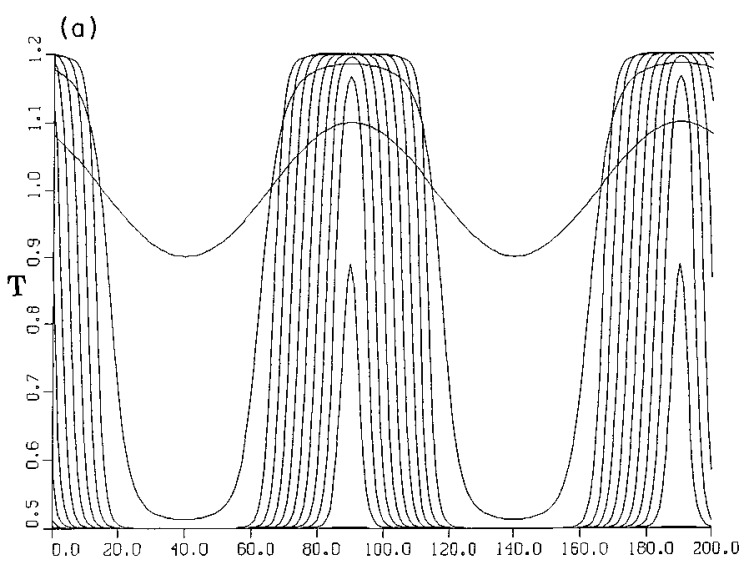

$\mathrm{m}$

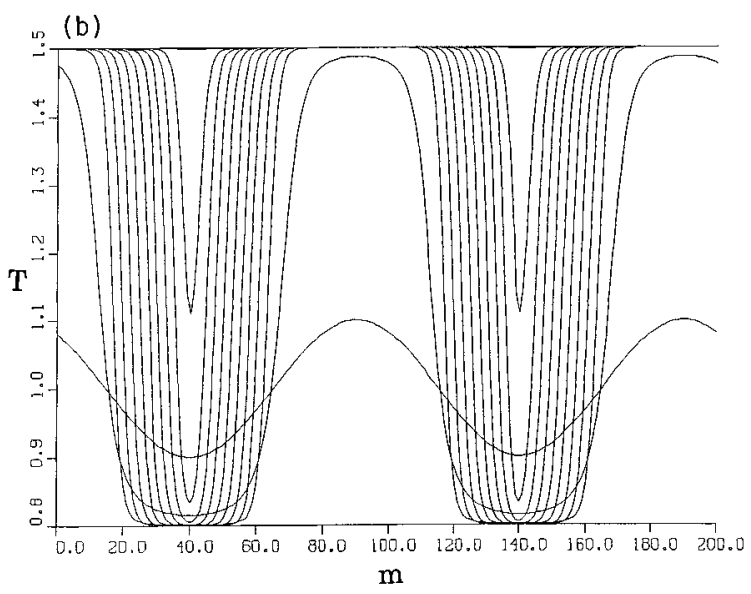

FIG. 3. Numerical solution of Eq. (26) with $u=T$. Bistable heat-loss function $\lambda_{L}(u, 1) \equiv \lambda(T)$ is taken from Eq. (43), and $\alpha=1$. The initial condition is a sinusoidal perturbation of the unstable equilibrium $T=1$ in the Lagrangian coordinate. The time interval between the temperature profiles is 20 . The values of the locally stable temperature equilibria are $T_{1}=0.5$ and $T_{2}=1.2$ [the higher-temperature phase survives, figure (a)], and $T_{1}=0.8$ and $T_{2}=1.5$ [the lower-temperature phase survives, figure (b)]. The Lagrangian length of the system $M=10^{5 / 2}$; the boundary conditions are periodic.

distance between them becomes comparable with the front width). Then, as numerical simulations show, these two fronts accelerate, "collide," and annihilate. Figures 3(a) and 3(b) show this process, as seen in a numerical example by Dimits and Meerson (1991). One can see that, after the annihilation, the plasma in the interaction region becomes uniform. The duration of the conductive stage is proportional to the typical distance between the interphase boundaries, that is, to the average initial size of the phases, $\langle L\rangle$.

As we have already mentioned, in the special case $I=0$ (area rule), a single front becomes standing rather than moving. If we neglect the (exponentially weak) interaction between the fronts, we would arrive at the conclusion that the segregated patterns will persist forever. In fact, this conclusion would be premature. The weak interaction between the fronts, considered in detail by Elphick et al. (1992), results in the fronts' eventual col- lisions and annihilation, so that the plasma ultimately becomes uniform again. However, relaxation to the uniform state takes a much longer time in this case [an exponentially long time with respect to the large parameter $\langle L\rangle$; see Elphick et al. (1992)].

Let us summarize this section. In an isobaric radiatively bistable plasma, radiative condensation can develop only on an intermediate time scale, which is of the order of the radiative cooling time. On a longer, thermal-conduction-related time scale (in our scaled units, it is of the order of $\langle L\rangle$ ), any intermediate radiative segregation is generally destroyed, and the plasma becomes uniform again. The parameters of the new uniform equilibrium correspond either to the hot phase or to the cool phase, depending on the sign of the integral (42). The only exception is provided by the case of a special area-rule value of the plasma pressure. In this case radiative condensation exists for a much longer (though still finite) time. However, realization of this special value of pressure in any natural isobaric system is highly unlikely.

\section{BISTABILITY IN CONFINED PLASMAS: TRIUMPH OF SEGREGATION}

Radiative condensation in confined plasmas is described, in planar geometry, by the reduced equations (15), (16), and (19) in Eulerian coordinates, or, alternatively, by Eqs. (21) and (22) in Lagrangian coordinates. These equations are valid for any heating-cooling function. Following Aranson et al. (1993), we shall concentrate on bistability. For a fixed $p$, the bistable heatingcooling function $\lambda(u, p)$, where $\lambda$ means either $\lambda_{L}$ or $\lambda_{E}=\lambda_{L} / u$, has an "unstable" root $u_{u}(p)$, surrounded by two "stable" roots, $u_{1}(p)<u_{u}(p)$ and $u_{2}(p)>u_{u}(p)$. The signs of the derivatives $\partial u_{1}(p) / \partial p, \partial u_{u}(p) / \partial p$, and $\partial u_{2}(p) / \partial p$ can be established in the general case (Aranson et al., 1993). Indeed, let us calculate $\partial u(p) / \partial p$ under the constraint $\lambda(u, p)=$ const. We have

$$
\left[\frac{\partial u(p)}{\partial p}\right]_{\lambda}=-\frac{(\partial \lambda / \partial p)_{u}}{(\partial \lambda / \partial u)_{p}}
$$

We have assumed that the spatially uniform equilibria are isochorically stable: $(\partial \lambda / \partial T)_{u} \geqslant 0$ for $u=u_{1}, u_{u}$, and $u_{2}$. Using the equation of state (4), we see that the derivative $(\partial \lambda / \partial p)_{u}$, entering Eq. (46), is equal to $u(\partial \lambda / \partial T)_{u}$. Therefore it is always non-negative, and we have $(\partial u(p) / \partial p)_{\lambda} \leqslant 0$ for the isobarically stable equilibria $u_{1}$ and $u_{2}$, and $(\partial u(p) / \partial p)_{\lambda} \geqslant 0$ for the isobarically unstable equilibrium $u_{u}$.

\section{A. Radiative stage}

Let us assume that the initial condition $u(m, 0)$ belongs to the intermediate-wavelength range and, as a first step, neglect conductivity. The set of equations (21) and (22) is more complicated than the single equation (26) and, even for a zero conduction, no analytic solution is generally possible. However, for a uniform initial 
condition, $u(m, 0)=u_{0}$, the analytic solution is quite simple and instructive. In this case, Eqs. (21) and (22) reduce to

$$
\begin{aligned}
& \dot{u}=\lambda_{L}(u, p)\left(\frac{u}{u_{0}}-1\right), \\
& \dot{p}=-\frac{\gamma p \lambda_{L}(u, p)}{u_{0}} .
\end{aligned}
$$

It follows from Eq. (47) that the specific volume remains unchanged: $u(t)=u_{0}$. We assume that the initial pressure $p_{0}$ is such that $u_{1}\left(p_{0}\right)<u_{2}\left(p_{0}\right)$. Then the pressure dynamics look as follows. If $u_{0}<u_{1}\left(p_{0}\right)$, the pressure will approach the value $p_{1}$ such that $u_{1}\left(p_{1}\right)=u_{0}$. If $u_{0}>u_{2}\left(p_{0}\right)$, the pressure will approach the value $p_{2}$ such that $u_{2}\left(p_{2}\right)=u_{0}$. If $u_{1}\left(p_{0}\right)<u_{0}<u_{u}\left(p_{0}\right)$, the pressure will approach either $p_{1}$ or $p_{u}$ such that $u\left(p_{u}\right)=u_{u}$. Finally, if $u_{u}\left(p_{0}\right)<u_{0}<u_{2}\left(p_{0}\right)$, then the pressure will approach either $p_{2}$ or $p_{u}$. In other words, the pressure always adjusts itself so that an arbitrary uniform initial condition becomes one of the three equilibria, described by the bistable heating-cooling function. This process takes a time of the order of unity (which in "physical" units corresponds to the radiative cooling time scale). As we already know, the "intermediate" equilibrium $u_{0}=u_{u}$ is unstable with respect to nonuniform perturbations; therefore all (slightly perturbed) uniform initial conditions, which belong to the "basin of attraction" of the unstable root $u_{u}$, will cause segregation of the plasma into the two phases 1 and 2.

Figure 4 shows an example of radiative segregation obtained by numerical solution of the Lagrangian equations (21) and (22) without the conduction term. In this example (and in other numerical simulations; see below), Aranson et al. (1993) chose the bistable heatingcooling function $\lambda_{L}(u, p)$ in the form of a cubic polynomial with respect to $u$ :

$$
\lambda(u, p)=\left[u-u_{1}(p)\right]\left[u-u_{u}(p)\right]\left[u-u_{2}(p)\right],
$$

with $u_{1}(p)=0.5 / p, u_{u}(p)=p$, and $u_{2}(p)=2 / p$. It is seen that a sharp front develops, the position of which does not coincide with the point where $u(m, 0)=u_{u}$. Instead, the front position is determined now by both the initial condition and the "length integral" (25). For example, in the case of a single front developing from a monotonic initial profile $u(m, 0)$, the front position $m_{f}$ is determined by one of the following relations:

$$
m_{f}=\frac{u_{2} M-L}{u_{2}-u_{1}} \text { or } m_{f}=\frac{L-u_{1} M}{u_{2}-u_{1}},
$$

depending on whether the function $u(m, 0)$ is increasing or decreasing with $m$, respectively. In Eulerian coordinates, the corresponding front positions in these two cases are $x_{f}=u_{1} m_{f}$ and $x_{f}=u_{2} m_{f}$, respectively. Note that the resulting specific volumes of the phases 1 and 2, entering Eq. (50), are determined by the final value of the plasma pressure. In the calculations presented in Fig. 4, Aranson et al. (1993) chose $M=50$ and the initial conditions $p(0)=1.0$ and $u(m, 0)=1.05+0.4 \cos (\pi m / M)$, so
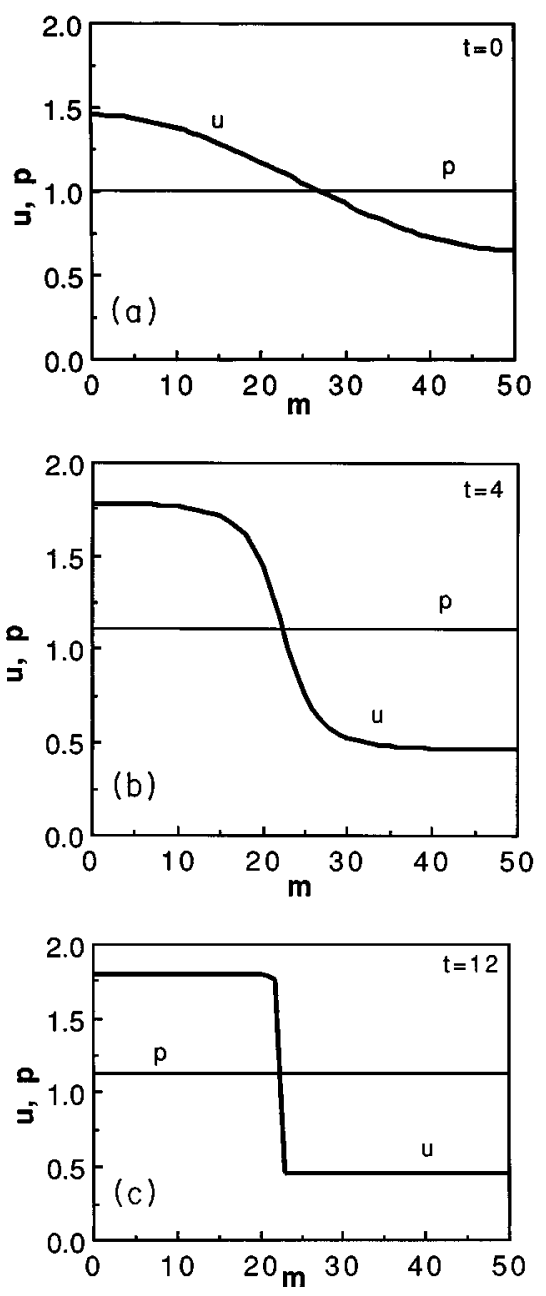

FIG. 4. Segregation developing at the radiative stage of the radiative condensation instability of a confined plasma. Equations (21) and (22), without the conduction term, were solved numerically for the bistable heating-cooling function $\lambda_{L}(u, p)$ from Eq. (49). Parameters are $u_{1}=0.5 / p ; u_{2}=2 / p ; u_{u}=p$; and $M=50$. Shown are (a) initial conditions, $u(m, 0)$ $=1.05+0.4 \cos (\pi m / M)$ and $p(0)=1.0$; and (b) and (c), subsequent evolution of $u(m, t)$ and $p(t)$ for scaled times $t=4$ and 12 , respectively.

that the system length is $L=52.5$. The second relation in Eq. (50) correctly predicts the position of the developing front in the Lagrangian coordinates, $m_{f} \approx 22.5$. In the Eulerian coordinates it corresponds to $x_{f} \approx 40.2$. In the case of two fronts, the length integral (25) predicts the distance between them.

Therefore, in the first, radiative stage of the radiative condensation instability, the plasma segregates into "cool drops" of phase 2 surrounded by the "hot gas" of phase 1 (or, alternatively, into "hot bubbles" of phase 1 surrounded by the "cool fluid" of phase 2). The slower conductive stage of the instability determines the final state of the system (evaporation of phase 2, condensation of phase 1, or their prolonged coexistence?) and requires the inclusion of thermal conduction. 


\section{B. Conductive stage}

We assume throughout this section that the size of the system is very large compared to the conductive Field length; that is $L \gg 1$ in the scaled equations. In addition, in the intermediate-wavelength limit, the front width, which is of the order of $\delta_{F}$ in "physical" units (and of the order of unity in the scaled variables), is very small compared to the typical distance between the fronts. Finally, in a long system, a typical front is located very far from the system's boundaries. Until exponentially large times, the form of the fronts and their dynamics can be investigated ignoring exponentially weak interactions between the fronts and the boundary effects. Therefore an "elementary" object during the conductive stage is an evolving single front. Our immediate aim is to exploit the strong inequality $L \gg 1$ and consider the statics and dynamics of a single front.

Let us return to Eqs. (15), (16), and (19) in Eulerian coordinates. (All the results in this section can be obtained in Lagrangian coordinates as well; however, we prefer to employ Eulerian coordinates at this stage, since some of the following equations will also be used in the two- and three-dimensional cases, in which Lagrangian coordinates become less convenient.) Let us start with an equilibrium, $\partial / \partial t=v=0$. We arrive at the following equation,

$$
\frac{d}{d x}\left(u^{\alpha} \frac{d u}{d x}\right)=p^{-\alpha} \lambda_{E}(u, p)
$$

which should be solved, in view of what has been mentioned above, for each front under the following boundary conditions: $u(x=-\infty)=u_{1}(p), u(x=+\infty)=u_{2}(p)$, and $d u / d x=0$ at both $-\infty$ and $+\infty$. When this solution is found, the alternative solution, for which $u(x=-\infty)=u_{2}(p)$ and $u(x=+\infty)=u_{1}(p)$, is obtained simply by putting $-x$ instead of $x$.

Multiplying Eq. (51) by $u^{\alpha} d u / d x$, integrating it once, and using the boundary conditions, we obtain

$$
\frac{1}{2}\left(\frac{d u}{d x}\right)^{2}=u^{-2 \alpha} p^{-\alpha} \int_{u_{1}(p)}^{u} u^{\alpha} \lambda_{E}(u, p) d u
$$

under the area-rule condition $I=0$, discussed in Sec. IV.B,

$$
\int_{u_{1}(p)}^{u_{2}(p)} u^{\alpha} \lambda_{E}(u, p) d u=0 .
$$

An equivalent criterion in the Lagrangian coordinates is obtained from Eq. (53) by replacing $\lambda_{E}$ by $\lambda_{L} / u$. Generally, Eq. (53) can be satisfied only for some discrete values of $p$. For example, if $\lambda_{E}(u, p)$ has the form of a cubic polynomial (49) and $\alpha=0$, these values of $p$ are the roots of the equation

$$
2 u_{u}(p)=u_{1}(p)+u_{2}(p)
$$

We assume that such a value of $p$ is unique and denote it by $p_{*}$, so that we can replace $p$ by $p_{*}$ in the equilibrium relation (52). Now we obtain from Eq. (52) the equilibrium solution in the following form: $x+$ const $=\sqrt{\frac{p_{*}^{\alpha}}{2}} \int_{u_{1}}^{u} u^{\prime \alpha} d u^{\prime} / \sqrt{\int_{u_{1}}^{u^{\prime}} \xi^{\alpha} \lambda_{E}\left(\xi, p_{*}\right) d \xi}$.

For example, the equilibrium solution can be conveniently found for the heating-cooling function (49) and $\alpha=0$ :

$$
u_{*}(x)=\frac{u_{1}+u_{2}}{2}+\frac{u_{2}-u_{1}}{2} \tanh \left[\frac{u_{2}-u_{1}}{2 \sqrt{2 p_{*}}}(x+\text { const })\right],
$$

where $u_{1}$ and $u_{2}$ should be evaluated at $p=p_{*}$ from Eq. (54).

As we noticed earlier, realization of the special case $p=p_{*}$, described by the area rule, is highly unlikely in any natural isobaric system. On the contrary, in confined plasmas with a "floating" pressure, the pressure can approach this special value $p_{*}$ with time, so that long-lived segregated states become possible (Aranson et al., 1993).

To understand this fact better, let us consider first the slow motion of a single front arising when the plasma pressure is close, but not equal, to the equilibrium pressure $p_{*}: p=p_{*}+\Delta p$, where $|\Delta p| \ll p_{*}$. Using the latter strong inequality, we can write

$$
\lambda_{E}(u, p) \approx \lambda_{E}\left(u, p_{*}\right)+\mu(u) \Delta p,
$$

where $\mu(u)=\partial \lambda_{E}(u, p) / \partial p$ calculated at $p=p_{*}$. Let us find the traveling-wave solutions of Eqs. (15) and (16): $u(x, t)=u(\xi), v(x, t)=v(\xi)$, where $\xi=x-\int{ }^{t} c\left(t^{\prime}\right) d t^{\prime}$ and $c(t)$ is the (slowly varying) front velocity. Equation (15) yields $v(\xi)=c+j u(\xi)$, where $j=$ const is the flux of material through the front in the reference frame where the front is at rest. We substitute this relation and Eq. (56) into Eq. (16) and obtain

$$
j \frac{d u}{d \xi}+\lambda_{E}\left(u, p_{*}\right)+\mu(u) \Delta p-p^{\alpha} \frac{d}{d \xi}\left(u^{\alpha} \frac{d u}{d \xi}\right)=0 .
$$

The term $\left(\gamma p_{*}\right)^{-1}(d \Delta p / d t)$ is of the next order of smallness (see below) and has therefore been neglected. At this stage, we notice that using the quantity $j$ is not very convenient, since, for a fixed front, the sign of $j$ depends on our choice of the direction of the coordinate axis (or, alternatively, for a fixed coordinate axis, on the sign of $d u / d \xi$ ). Instead, we can introduce the projection $j_{n}=\mathbf{j} \cdot \mathbf{n}$ of the flux vector $\mathbf{j}$ onto the unit vector $\mathbf{n}$, normal to the front and directed, for concreteness, from phase 1 to phase 2 . In the planar case, with which we are working now, such a definition makes the subsequent equations independent of the sign of $d u / d \xi$, which is quite convenient. Moreover, this definition becomes very convenient in the two- and three-dimensional cases, as will be seen in the subsequent section.

Let us multiply Eq. (57) by $u^{\alpha} d u / d \xi$ and integrate it over $\xi$ from $-\infty$ to $+\infty$. Because of the smallness of $j$, we can replace $u^{\alpha}(d u / d \xi)^{2}$ under the integral by its equilibrium value from Eq. (52). Using the single-front boundary conditions and Eq. (53), we arrive at the following linear relation between $j_{n}$ and the small pressure mismatch $\Delta p$ : 


$$
j_{n}=-g \Delta p,
$$

where

$g=p_{*}^{\alpha / 2} \int_{u_{1}}^{u_{2}} u^{\alpha} \mu(u) d u / \int_{u_{1}}^{u_{2}} \sqrt{2 \int_{u_{1}}^{u} \eta^{\alpha} \lambda_{E}\left(\eta, p_{*}\right) d \eta} d u$.

Equation (58) implies that a pressure mismatch $\Delta p$ causes a plasma flow through the front. Furthermore, the flux of material from, say, phase 1 into phase 2 is the same for every front, as long as the fronts are sufficiently far from each other and from the boundaries. Defining the normal plasma velocity components in phases 1 and 2 and the normal component of the front velocity $\mathbf{c}$ in the same way as before, we have

$$
v_{1 n}=c_{n}-g u_{1} \Delta p, v_{2 n}=c_{n}-g u_{2} \Delta p,
$$

for phase 1 and 2 , respectively.

The $g$ factor in Eq. (59) can be conveniently calculated for the cubic polynomial $\lambda_{E}$ and $\alpha=0$. The result is the following:

$$
g=\frac{1}{\sqrt{2}}\left(2 \frac{d u_{u}}{d p}-\frac{d u_{1}}{d p}-\frac{d u_{2}}{d p}\right),
$$

which should be evaluated at $p=p_{*}$.

Now we shall use Eq. (58) to simplify the evolution equation for the pressure, Eq. (19), in the case of $|\Delta p| \ll p_{*}$. One could start directly from Eq. (19) and calculate the integral, using an asymptotic expansion of the integrand with respect to the small parameter $|\Delta p| / p_{*}$. We shall present here an alternative derivation, directly using the mass and length conservations.

Consider a radiatively segregated plasma, in which the pressure is already close to the equilibrium value, $|\Delta p| \ll p_{*}$. The system consists of regions of phases 1 and 2 , and the mass transfer between neighboring regions proceeds according to the local relation (58). Neglecting the front widths, we have

$$
L_{1}+L_{2}=L, M_{1}+M_{2}=M,
$$

where indices 1 and 2 correspond to the regions of phases 1 and 2, respectively. If $N$ is the total number of interphase boundaries (fronts), then the net mass rate of "evaporation" is $d M_{2} / d t=j_{n} N$, while the net mass rate of "condensation" is $d M_{1} / d t=-j_{n} N$. Recalling that $L_{1}(t)=M_{1}(t) u_{1}(t)$ and $L_{2}(t)=M_{2}(t) u_{2}(t)$, using relations (58) and (62), and noticing that $d u_{1,2} / d t=\left(\partial u_{1,2} / \partial p\right)_{\lambda}(d p / d t)$, we arrive at the following evolution equation for the pressure:

$$
\begin{aligned}
\frac{d p}{d t}= & g\left(u_{2}-u_{1}\right)^{2}\langle u\rangle\left(p-p_{*}\right) /\left[\langle L \rangle \left(\left(u_{2}-\langle u\rangle\right) \frac{\partial u_{1}}{\partial p}\right.\right. \\
& \left.\left.+\left(\langle u\rangle-u_{1}\right) \frac{\partial u_{2}}{\partial p}\right)\right]
\end{aligned}
$$

(Aranson et al., 1993), where $\langle L\rangle=L / N$, while $\langle u\rangle=L / M=$ const is determined by the initial condition $u(x, 0)$ :

$$
\langle u\rangle^{-1}=\frac{1}{L} \int_{0}^{L} \frac{d x}{u(x, 0)} .
$$

Generally, $u_{1,2}$ depend on $p$. In this case $\partial u_{1,2} / \partial p$ has been shown previously to be negative. The expressions $g, u_{2}-\langle u\rangle$, and $\langle u\rangle-u_{1}$ are all positive. Therefore Eq. (63) predicts relaxation of the pressure to the equilibrium value, $p \rightarrow p_{*}$. The quantities $u_{1}, u_{2}, \partial u_{1} / \partial p$, and $\partial u_{2} / \partial p$, entering Eq. (63), should be evaluated at $p=p_{*}$. Therefore the pressure relaxation, described by Eq. (63), is exponential in time: $\Delta p \propto \exp [-t /(C\langle L\rangle)]$, where the constant $C$ is determined from Eq. (63). If neither large nor small factors are introduced by the roots of heating-cooling function, the constant $C$ is of the order of unity, so that the (scaled) $e$-folding time of the pressure relaxation is of the order of $\langle L\rangle$, which is the large parameter of the theory. Equation (63) is valid in Lagrangian coordinates as well. One need only use the simple relation between $\lambda_{L}$ and $\lambda_{E}, \lambda_{L}=u \lambda_{E}$, when calculating the $g$ factor.

In deriving Eq. (63), we assumed that the number of fronts $N$ is constant. In the beginning of the conductive stage, this assumption is generally not correct, and fast motions, "collisions," and "annihilations" of the fronts occur, so that irregularities in the pressure dynamics can be expected. However, towards the end of the conductive stage, when $\Delta p$ is already sufficiently small, the front motion decelerates significantly and the assumption of a constant $N$ becomes true as well, which makes the final pressure relaxation to $p_{*}$ monotonic (and exponential).

Since the average distance between the fronts $\langle L\rangle$ proves to be an important characteristic of the dynamics, a few words seem to be in order on how to determine it. In simple cases, $\langle L\rangle$ is determined by the form of the Fourier spectrum of the initial perturbation. For example, if the Fourier spectrum has a pronounced maximum at some wavelength (belonging to the intermediate-wavelength limit), this wavelength will normally define $\langle L\rangle$. An extreme limit would be a monochromatic initial perturbation, as in Fig. 4, when $\langle L\rangle$ is simply equal to a half of the perturbation wavelength. On the contrary, if the initial perturbation spectrum has the form of broadband noise with no preferential wave number, an a priori determination of $\langle L\rangle$ becomes difficult, and numerical solution of the reduced equations is required (see below).

Therefore the mass conservation and length constancy of the system do provide a universal mechanism for persistent radiative condensation: the plasma pressure approaches the special value $p_{*}$ for which the fronts stop and the segregated patterns exist for a very long time. However, since the analytical theory is unable to describe the "violent" part of the conductive stage, when the pressure mismatches are still large, and fast front motions, collisions, and annihilations occur, we shall also discuss the results of numerical simulations with this system (Aranson et al., 1993). 


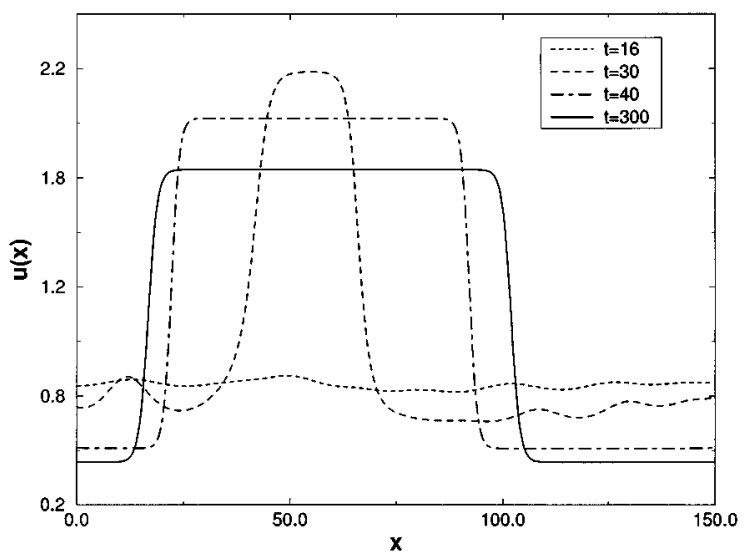

FIG. 5. Development of planar steady-state patterns in the process of radiative condensation. Shown are the dynamics of the spatial profile of the specific volume $u(x, t)$, found by the numerical solution of Eqs. (15), (16), and (19). The heatingcooling function $\lambda_{E}(u, p)$ is taken from Eq. (49), with $u_{1}=0.5 / p, u_{2}=2 / p$, and $u_{u}=p$; parameter $\alpha=0$ and $\gamma=5 / 3$. The initial condition for $u(x, t)$ represents broadband noise with a small amplitude around $u_{0}=0.8$. The initial velocity is zero, and the initial pressure is 1.0. The evolution is shown in four successive (scaled) time moments: 16 (short dashes), 30 (medium dashes), 40 (long-and-short dashes), and 300 (solid line).

\section{Numerical simulations}

Aranson et al. (1993) employed the bistable heatingcooling function from Eq. (49) and solved numerically both the Eulerian reduced equations (15), (16), and (19), and the Lagrangian reduced equations (21) and (22).

For the Eulerian simulations, Aranson et al. (1993) put $\alpha=0\left[K(T)=K_{0}\right]$ and $\gamma=5 / 3$ and chose $\lambda_{E}(u, p)$ of the form given in Eq. (49). The boundary conditions were the same as in their theory: $\partial u / \partial x=0$ at $x=0$ and $x=L$. The initial velocity was always taken to be zero.

For the set of equilibria $u_{1}=1 /(2 p), u_{u}=p$, and $u_{2}=2 / p$, the correct order of the equilibria, $u_{1}<u_{u}<u_{2}$, is provided for $1 / \sqrt{2}<p<\sqrt{2}$, while the equilibrium (area-rule) value for the pressure is $p_{*}=\sqrt{5} / 2$. The initial condition for the specific volume $u(x, 0)$ had the form of very small-amplitude broadband noise with an average value $\langle u\rangle$ which belonged to the interval $\left(u_{1}(p), u_{2}(p)\right)$ for the initial value of the pressure, $p(0)$. The chosen initial condition included both intermediate- and short-wavelength Fourier components.

Aranson et al. (1993) varied the system length and the initial conditions for the specific volume and pressure. A typical example of the radiative condensation dynamics is shown in Fig. 5-where the profile of the specific volume of the plasma is presented at four successive moments in time, 16, 30, 40, and 300-and in Fig. 6, where the pressure evolution is shown, starting from the initial values $\langle u\rangle=0.8$ and $p(0)=1.0$. In this example, the (scaled) total length of the system $L$ was taken to be 150. The (scaled) total mass of the gas was therefore $M=150 / 0.8=187.5$. It is seen that, in the beginning, the

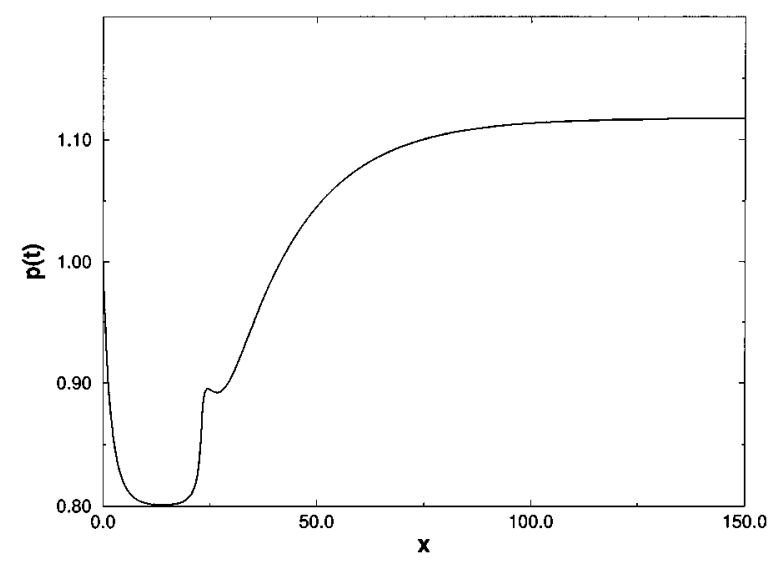

FIG. 6. Plasma pressure vs time, corresponding to Fig. 5.

pressure rapidly drops to $\langle u\rangle=0.8$. In other words, the initial condition for the specific volume becomes a perturbation around an unstable equilibrium, as predicted by the "uniform" equations (21) and (22).

Then the linear stage of the radiative condensation instability starts. The perturbations with too short wavelengths are strongly damped in agreement with Eq. (5), so that they are already absent in Fig. 5. (Noteworthy in this stage is the U-shaped valley around $t=15$ in Fig. 6 . It is explained by the fact that pressure variations vanish in the linear theory with respect to $u$ and $v$, and appear only in the second order in the perturbation amplitudes.) After that the instability develops nonlinearly, causing segregation (around $t=30$ ), while the plasma pressure rapidly grows. Since the initial perturbation amplitudes are very small in this example, the radiative stage takes a somewhat longer time.

After a transient process, a steady coherent pattern forms, which consists of a lower-density, highertemperature "bubble" surrounded by two higherdensity, lower-temperature "drops." The equilibrium phase boundaries represent two symmetric fronts, described in Sec. V.B. The pattern reaches its steady state around $t=80$ to 100 radiative times. The distance between the fronts coincides with that predicted by the length and mass conservation. The plasma pressure finally relaxes monotonically to a value very close to $p_{*}=\sqrt{5} / 2 \approx 1.12$, as predicted by the theory. For larger scaled times (up to $t=300$ ), the profile of $u$ and the plasma pressure do not show any change.

Aranson et al. compared the final stage of the pressure relaxation, found numerically, with that predicted by Eq. (63). To this end, they calculated the coefficients entering Eq. (63) for the chosen $\lambda_{L}(u, p)$, evaluating them at $p=p_{*}=\sqrt{5} / 2$. Equation (59), for the $g$ factor, gives $g=2 \sqrt{2}$. The only quantity that cannot be found analytically is the final number of fronts; therefore Aranson et al. (1993) took it from the numerical results: $N=2$, which gives $\langle L\rangle=150 / 2=75$. Substituting all the coefficients into Eq. (63), they obtained $d p / d t$ $\approx-0.057\left(p-p_{*}\right)$, so that the theoretical prediction of the relaxation rate is 0.057 . Figure 7 shows, on a logarithmic scale, the final stage of the pressure relaxation 


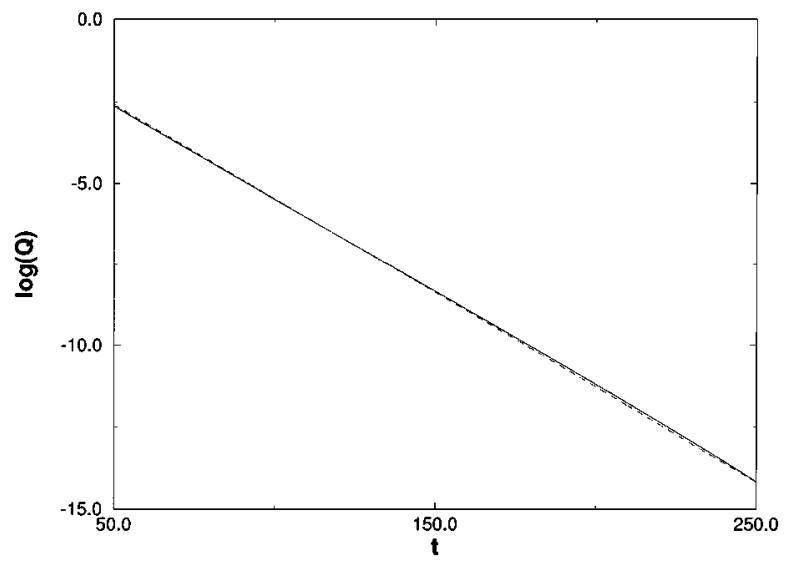

FIG. 7. Final pressure relaxation, corresponding to Fig. 6. The solid line shows the natural logarithm of $Q$ vs time found numerically ( $Q$ is the absolute value of the difference between the pressure and its equilibrium value $p_{*}$ ). The dashed line is a linear approximation of this dependence.

found numerically and its linear approximation. It is seen that the relaxation is indeed exponential, starting from $t \approx 50$. The relaxation rate found numerically is approximately 0.058 , so that the theory predicts the relaxation rate with a very good accuracy.

For the numerical solution of the Lagrangian equations (21) and (22), Aranson et al. (1993) took $\alpha=1$ (a model thermal diffusivity, proportional to the temperature) and chose the heating-cooling function from Eq. (49), but taken for $\lambda_{L}(u, p)$ instead of for $\lambda_{E}$, as in the previous example. The results closely resembled those shown in Figs. 5-7: formation of long-lived patterns, accompanied by pressure relaxation to the special value determined by the area rule for a single front.

\section{Intermediate-wavelength limit: summary and discussion of the planar case}

We have considered the dynamics of planar radiative condensation in confined plasmas in the intermediatewavelength limit. We have described a universal mechanism by which long-lived coherent patterns can develop in the process of radiative condensation instability. Long-time two-phase coexistence, generally impossible in isobaric systems, becomes possible here because the time-dependent plasma pressure can approach the arearule value for which individual fronts separating the phases become standing rather than moving. Numerical simulations strongly support and even reinforce these theoretical predictions, removing the limitation of small pressure mismatches used in the analytic theory.

So far, we have been considering two stages of the dynamics. During the first radiative stage, the duration of which is determined by the radiative cooling time, the plasma segregates into two thermal phases with sharp boundaries (fronts) between them. The plasma pressure varies significantly during this stage. The mass ratio of the two phases, the number and location of homogeneous regions in each of them, and the gas pressure at the end of the radiative stage depend on the initial conditions. Then the much slower, conductive stage starts, during which the phase interfaces (fronts) move with velocities of the order of $\delta_{F} \mathscr{L}_{0}$ (which is unity in the scaled equations). Some of the fronts can collide and annihilate, leading to irregular pressure dynamics. At the end of the conductive stage, the fronts steadily decelerate and finally stop, with $p$ approaching $p_{*}$ exponentially. This implies development of a long-lived stratification, or pattern formation, via the radiative condensation instability. The typical duration of the second phase is of the order of the average distance between the fronts, divided by the typical front speed. In the scaled units, this duration is of the order of $\langle L\rangle$, which is the large parameter of the theory. It is important that, for a given heating-cooling function, the equilibrium ratio of masses of the two phases, which set in at the end of the conductive stage, depends only on the total plasma mass and the system length. The equilibrium plasma pressure, developing towards the end of the conductive stage, is determined by the area rule and depends only on the form of the heating-cooling function.

We should stress that persistent segregation, developing in confined systems, is essentially due to a nonlocal constraint (in our case, total mass conservation) imposed on the system. This constraint leads to a (nonlocal) feedback, arresting the motion of the interface boundaries (traveling fronts). Similar mechanisms of persistent one-dimensional pattern formation via a nonlocal constraint appear in many other applications in physics and chemistry (Nedospasov and Khait, 1979; Pismen, 1979; Barelko et al., 1981; Gurevich and Mints, 1987; Elmer, 1992; Middya et al., 1993), and their mathematical models have been recently extended to higher dimensions (Rubinstein and Sternberg, 1992; Meerson and Sasorov, 1996).

An interesting question concerns the character of the final, third stage of the evolution of this system, when the weak interfront interaction and boundary effects become important. This question is of academic interest for sufficiently large systems (such as many interstellar clouds). However, for smaller systems (closer to the short-wavelength limit), this stage becomes attainable. This question has not been addressed authoritatively yet, but some simple predictions can be made (Aranson et al., 1993). First, because of the mass conservation, no complete relaxation to a uniform state is possible during the third, "superlong" stage, in contrast to the isobaric case studied by Elphick et al. (1992). Therefore some (at least rudimentary) pattern structure must exist "forever." On the other hand, a family of exact equilibrium solutions of Eq. (51) exists in an unbounded medium, which represent nonlinear periodic standing temperature (and density) waves (Meerson, 1989). Solutions consisting of an integer number of "segments" of such temperature waves and satisfying the no-flux boundary conditions can always be constructed (unless the system is too short and the radiative condensation instability is suppressed). These solutions provide all possible candidates for the final state, and one can assume that the 
"simplest" of them, containing only one front, will generally be stable and therefore realizable as $t \rightarrow \infty$. The expected (scaled) duration of the third stage must be of the order of $\exp [O(L)]$, that is, exponentially large with respect to the ratio of the system length to the conductive Field length, the large parameter of the theory.

In two- and three-dimensional cases, the conductive stage becomes more complex as new factors-the transverse stability and curvature of the fronts-affect the dynamics. These and related problems will be reviewed in Sec. VII. In the following section we continue our treatment of planar radiative condensation, but this time we shall consider the long-wavelength limit.

\section{RADIATIVE CONDENSATIONS IN THE LONG-WAVELENGTH LIMIT}

\section{A. Unlimited instability and "anomalous" gas dynamics}

In the long-wavelength limit, the planar dynamics of radiative condensation is described by Eqs. (28) and (29) with a nonmonotonic "effective" pressure $p=P(\rho)$. Let us first consider quite an interesting limit of the problem, when the unstable density range is very large. Meerson and Sasorov (1987) formally extended this density region to infinity and, neglecting the viscosity, arrived at a model problem of an ideal, everywhere unstable flow of a gas with a negative compressibility. They approximated the unstable part of $p=P(\rho)$ as follows:

$$
P(\rho)=A+B \rho^{1-\sigma},
$$

where $A>0, B>0$, and $\sigma>1$ are constants. The presence of the three fitting parameters $A, B$, and $\sigma$ makes it possible to obtain an accurate approximation for $P$ in the unstable density range in the most widely different cases. Introduce the scaled variables $\tilde{x}=x / L$, $\tilde{t}=c t / L, \tilde{v}=v / c$, and $\tilde{\rho}=\rho / \rho_{0}$, where $\rho_{0}$ is the characteristic value of the gas density and $c^{2}=(\sigma-1) B \rho_{0}^{-\sigma}$. The quantity $c$ is the characteristic gasdynamic velocity of the problem. Omitting the tildes, rewrite Eq. (29) in the form

$$
\frac{\partial v}{\partial t}+v \frac{\partial v}{\partial x}-\rho^{-(1+\sigma)} \frac{\partial \rho}{\partial x}=0
$$

while retaining the form for Eq. (28) in the scaled variables. Like any other planar nonviscous compressible gas flow, the flow described by Eqs. (28) and (66) can be reduced, by means of either a Legendre or hodograph transformation, to a linear partial differential equation of the second order (see, e.g., Landau and Lifshitz, 1987).

The set of equations (28) and (66) with different particular values of parameter $\sigma$ has arisen in a whole series of works in other contexts [such as tearing, Buneman and parametric instabilities of a plasma, various modulational instabilities, etc.; for an extensive review, see Trubnikov and Zhdanov (1987), where more than 20 physical examples are listed]. These works go back to Chaplygin, who studied a particular example of such a problem in 1896 [Chaplygin's selected works were pub- lished in the 1970s; see Chaplygin (1976)]. The peculiarity of the unstable flow (28) and (66) manifests itself in the fact that the above-mentioned second-order linear partial differential equation proves to be of the elliptic type (see below), in contrast to the "usual" hyperbolic type typical for "normal" (that is, everywhere stable) gasdynamics. It is well known that the initial-value problem for elliptic equations is ill posed in the sense that the solution does not exist for $t \rightarrow \infty$. As it turns out, the solution always develops a singularity in a finite time. Physically, there are two possible types of singularities in the system (28) and (66). In the first, the plasma density goes to zero (correspondingly, the temperature goes to infinity). We call this type of singularity explosive rarefaction. In the second type, the plasma density goes to infinity, while the temperature goes to zero, which is called explosive condensation, or collapse. The singular character of the solution implies that the "advective" nonlinearities, present in Eqs. (28) and (66), are unable to stop the instability, and they even accelerate it.

Meerson and Sasorov (1987) and Trubnikov and Zhdanov (1987) investigated the idealized problem (28) and (66) analytically for an arbitrary real value of the parameter $\sigma>1$. They employed a hodograph transformation, which consists in going over from the functions $\rho(x, t)$ and $v(x, t)$ to the functions $x(\rho, v)$ and $t(\rho, v)$, where $\rho$ and $v$ are now considered as the independent variables. The equations for $x(\rho, v)$ and $t(\rho, v)$ then take the form

$$
\frac{\partial}{\partial \rho}(x-v t)=\rho^{-(\sigma+2)} \frac{\partial}{\partial v}(t \rho), \frac{\partial}{\partial v}(x-v t)=-\frac{\partial}{\partial \rho}(t \rho),
$$

whence

$$
\begin{aligned}
& \left(\frac{\partial^{2}}{\partial \rho^{2}}+\frac{1}{\rho^{\sigma+2}} \frac{\partial^{2}}{\partial v^{2}}\right)(t \rho)=0, \\
& x-v t=\int\left(\frac{1}{\rho^{\sigma+2}} \frac{\partial(t \rho)}{\partial v} d \rho-\frac{\partial(t \rho)}{\partial \rho} d v\right) .
\end{aligned}
$$

The integrand of Eq. (69) in the $(\rho, v)$ plane is, according to Eq. (67), a total derivative.

Because of its linearity, Eq. (68) admits, in principle, the possibility of construction of a general solution. This is clearly seen if we make the change of variables $t(\rho, v)=\rho^{1 / 2} \Phi\left(\rho^{-\sigma / 2}, \sigma v / 2\right)$. Then, for the function $\Psi=\Phi(r, z) \exp (i \phi / \sigma)$, by means of Eq. (68), we obtain the Laplace equation, $\nabla^{2} \Psi=0$, in the cylindrical coordinates $r, \phi, z$. The well-known powerful methods of solution of the Laplace equation make it possible to obtain a large set of physically interesting solutions and to investigate different regimes of flow of the unstable gas. A very useful technique here turns out to be transforming to different coordinate systems, such as spherical and toroidal, in the Laplace equation, which yields a rich selection of solutions with different properties. Solving the Laplace equation, we obtain the function $t(\rho, v)$. Then we find $x(\rho, v)$ from Eq. (69). Inverting the algebraic relations for $t(\rho, v)$ and $x(\rho, v)$, we obtain the solutions for the density and velocity. Using this technique, 


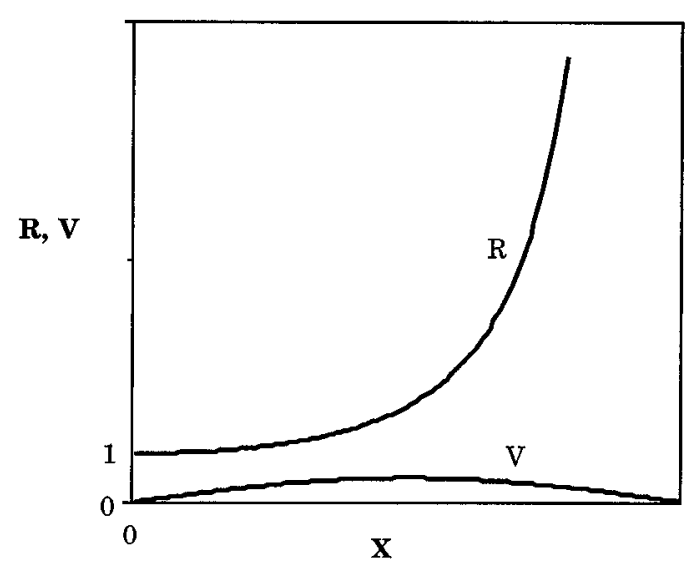

FIG. 8. Spatial structure of the selected self-similar solutions (70) for the gas density $(R)$ and velocity $(V)$, describing explosive rarefaction of an "unlimitedly" unstable plasma.

Meerson and Sasorov (1987) and Trubnikov and Zhdanov (1987) obtained a number of exact solutions of the problem, both for explosive rarefaction and for collapse.

Let us start with the case of explosive rarefaction. Because of the (very strong) instability, the form of the solution is very sensitive to the initial and boundary conditions. We are interested, most of all, in the behavior of the gas flow near the singularity $\rho \rightarrow 0$ that (we shall assume) arises in the plane $x=0$ at $t=0$. Among different solutions [including a family of self-similar solutions which can be found directly from Eqs. (28) and (66)], there is a special one that corresponds to a smallamplitude single-mode perturbation of the density and velocity at $t \rightarrow-\infty$. Near the time moment of singularity, $t=0$, the selected solution looks like

$$
\rho=(-t)^{2 / \sigma} R(x), v=(-t)^{-1} V(x),
$$

where

$$
\begin{aligned}
& 1+(\sigma / 2)^{2} R^{\sigma} V^{2}=R^{\sigma^{2} /(\sigma+2)}, \\
& \int_{1}^{R} \frac{d R}{R} \frac{4 R^{-2 \sigma /(\sigma+2)}+\left(\sigma^{2}-4\right) R^{-\sigma}}{(\sigma+2)\left(R^{-2 \sigma /(\sigma+2)}-R^{\sigma}\right)^{1 / 2}}=2 x
\end{aligned}
$$

(Meerson and Sasorov, 1987).

Notice that the density vanishes, while the velocity of the gas becomes infinitely large, on a whole segment of the $x$ axis simultaneously. In Eqs. (70)-(72), the length of the segment is already specified. In reality, it is uniquely determined by the wavelength of the initial perturbation; therefore the form of Eqs. (70)-(72) presumes a specific choice of the wavelength (see Meerson and Sasorov, 1987 for details). The behavior of the functions $R(x)$ and $V(x)$ is depicted in Fig. 8. For example, in the particular case $\sigma=2$, one obtains simple solutions $R=\cos ^{-2} x$ and $V=(1 / 2) \sin 2 x$, first found by Belova et al. (1980) in another context.

The second type of singularity (collapse) can be conveniently studied with the following exact solutions of Eqs. (28) and (66) (Meerson and Sasorov, 1987):

$$
\begin{aligned}
& x=-\frac{a_{1} v}{\rho}+\frac{2}{3} a_{2} v^{3}-\frac{2 a_{2}}{\sigma-1} \frac{v}{\rho^{\sigma}}, \\
& t=-\frac{a_{1}}{\rho}+a_{2} v^{2}-\frac{2 a_{2}}{\sigma^{2}-1} \frac{1}{\rho^{\sigma}},
\end{aligned}
$$

where $a_{1}, a_{2}>0$ are constants. Of course, these relations must be inverted to yield the density and velocity. Since $\sigma>1$, we can neglect the last terms in Eqs. (73) and (74) as $\rho \rightarrow \infty$. Then we arrive at a well-known problem of the inertial motion of the gas, when the role of the pressure gradient is negligibly small (flow of a gas of noninteracting particles). Arnold et al. (1981) showed that the gas flow (73) and (74), with the last terms neglected, corresponds to the only structurally stable singularity of the flow of a gas of noninteracting particles. This result makes the solution (73) and (74) the selected one for collapse.

The fact that, when collapse develops, the gas flow proceeds in the same way as in a gas of noninteracting particles is physically fairly obvious, since for the chosen approximation of $P(\rho)$ in the form (65) we have $P(\rho) \rightarrow$ const as $\rho \rightarrow \infty$ (and, correspondingly, $\nabla p \rightarrow 0)$. The latter circumstance also holds in the threedimensional case, and this makes it possible to detect a similarity between the radiative condensation collapse, considered here, and long-wavelength gravitational collapse (Zel'dovich and Novikov, 1983). In the latter case, Zel'dovich predicted the formation of planar structures-the celebrated "Zel'dovich's pancakes," and a set of shock waves attached to these structures. In the problem of a three-dimensional radiative collapse, one can also expect flattening and formation of a planar condensation, including shock waves (Meerson and Sasorov, 1987). [Moreover, in his very elegant paper, Sasorov (1988) showed that the flattening persists even in the intermediate-wavelength limit, though the mathematics he used was quite different. Sasorov neglected thermal conduction and considered the nonlinear evolution of an unlimitedly unstable plasma under conditions of isobaricity. Choosing specific initial conditions, he was able to formally reduce the problem to that of the dynamics of the components of the deformation tensor of a gaseous ellipsoid, thoroughly investigated by Bogoyavlenskii (1985). Under these conditions, Sasorov (1988) showed that a three-dimensional density perturbation develops, in a finite time, into a two-dimensional structure ("Sasorov's pancake").]

The analytical solutions described here in Sec. VI.A are useful as approximations at some stage of the dynamics, if the unstable density region is very broad. Of course, in reality no singularities will develop, as the plasma density eventually enters the regions of a normal gasdynamics, that is, positive compressibility (see Fig. 2). Therefore we have to consider the complete problem, Eqs. (28) and (29), of the flow of a gas with an alternatesign compressibility. Such a problem was considered by Meerson, Steele et al. (1993), and we review the results of their work in Sec. VI.B. 


\section{B. Normal-anomalous gasdynamics and bistability}

The similarity between the problem of the flow of a gas with an alternate-sign compressibility and the problem of the isothermal dynamics of the van der Waals gas (see Sec. III) gives a hint about possible final states. In the van der Waals gas, the instability results in segregation of the overcooled vapor into two phases: the liquid and the "normal" vapor, which can coexist in an inhomogeneous state (Kittel and Kroemer, 1980). This means that bistability must exist in the long-wavelength limit, too. Therefore we shall start with the possible spatially nonuniform equilibria of the long-wavelength reduced equations, described by the conditions $v=0$ and $\partial / \partial t=0$, from which follows $\partial p / \partial x=0$.

For such an equilibrium the density "jumps" from, say, $\rho_{1}$ to $\rho_{2}>\rho_{1}$ at some point $x_{0}$, while the pressure remains uniform: $P\left(\rho_{1}\right)=P\left(\rho_{2}\right)$. This equilibrium [the so-called contact discontinuity; see Landau and Lifshitz (1987), p. 321] is obviously impossible to attain if both $\rho_{1}$ and $\rho_{2}$ belong to the same rising or falling branches of the $P(\rho)$ curve [just as it is impossible in the case of a "normal," that is, growing, $P(\rho)$ ]. However, it becomes possible when $\rho_{1}$ and $\rho_{2}$ belong to different rising branches of $P(\rho)$, while $P\left(\rho_{1}\right)=P\left(\rho_{2}\right)$, as at the points $\mathrm{A}$ and $\mathrm{B}$ in Fig. 2. In the problem of the long-wavelength radiative condensation, such a contact discontinuity represents an equilibrium boundary between the cool and dense plasma on one side and the hot and dilute plasma on the other. In the van der Waals model, it is the equilibrium boundary between the coexisting liquid drop and normal vapor. Notice that this static structure has a zero thickness, and the viscosity does not play any role. Therefore we indeed have bistability in the longwavelength regime.

A natural generalization of discontinuous equilibria includes propagating shock waves, which are described by traveling-wave solutions of Eqs. (28) and (29). As the plasma velocity in this solution is nonzero, the viscosity determines a finite width of the shock front.

Looking for traveling-wave solutions, $\rho(x, t)=\rho(\xi)$ and $v(x, t)=v(\xi)$, where $\xi=x-c t$ and $c$ is the wave speed, we arrive at the following equations:

$$
\begin{aligned}
& \rho(v-c)=\rho_{1}\left(v_{1}-c\right)=\rho_{2}\left(v_{2}-c\right), \\
& \rho(v-c) \frac{d v}{d \xi}=-\frac{d P}{d \xi}+\eta \frac{d^{2} v}{d \xi^{2}},
\end{aligned}
$$

with the boundary conditions $\rho=\rho_{2}, v=v_{2}$ at $\xi=-\infty$, and $\rho=\rho_{1}, v=v_{1}$ at $\xi=+\infty$. Using Eq. (75) and integrating Eq. (76) once, taking account of the boundary conditions, we obtain

$$
\eta c_{0} \rho_{2} \frac{d}{d \xi}\left(\frac{1}{\rho}\right)=-P(\rho)+P_{2}-c_{0}^{2} \frac{\rho_{2}^{2}}{\rho}+c_{0}^{2} \rho_{2},
$$

where

$$
\left(v_{2}-c\right)^{2}=c_{0}^{2}=\frac{P_{2}-P_{1}}{\rho_{2}-\rho_{1}} \frac{\rho_{1}}{\rho_{2}},
$$

$$
\left(v_{1}-c\right)^{2}=\frac{P_{2}-P_{1}}{\rho_{2}-\rho_{1}} \frac{\rho_{2}}{\rho_{1}},
$$

and $P_{1,2}=P\left(\rho_{1,2}\right)$. Furthermore, from Eqs. (78) and (79),

$$
\left(v_{1}-v_{2}\right)^{2}=\frac{\left(P_{2}-P_{1}\right)\left(\rho_{1}+\rho_{2}\right)}{\rho_{1} \rho_{2}} .
$$

It follows from any of Eqs. (78)-(80) that, if $\rho_{2}>\rho_{1}$, then $P_{2}>P_{1}$. Formally speaking, the same inequality, as well as relations (77)-(80), holds also for "normal" gasdynamics (Landau and Lifshitz, 1987). An important difference, however, is the fact that the inequality $P_{2}>P_{1}$ can be achieved now not only on one rising branch of the $P(\rho)$ curve, but also on its different rising branches. Therefore shock waves with a monotonic density profile, but a nonmonotonic pressure profile, are possible. The front of such an anomalous shock wave represents a narrow unstable density region confined by two stable regions of higher and lower density. As a consequence, a small pressure difference $P_{2}-P_{1}$ in the anomalous shock wave may correspond to a very large density ratio $\rho_{2} / \rho_{1}$, which results in a small value of $c_{0}$ and therefore a narrow shock front [see Eq. (77), where the shock front width is seen to be proportional to $c_{0}$ ].

Another important difference between the "normal" and "anomalous" shocks becomes clear if we try to integrate Eq. (77) and find the density profile $\rho=\rho(\xi)$ of the shock wave. Introducing the specific volume $u=\rho^{-1}$, we rewrite Eq. (77) as

$\frac{\eta c_{0}}{u_{2}} \frac{d u}{d \xi}=F(u) \equiv P_{2}+\frac{P_{2}-P_{1}}{u_{2}-u_{1}}\left(u-u_{2}\right)-P(1 / u)$,

where $u_{1,2}=\rho_{1,2}^{-1}$. The function $F(u)$ vanishes at $u=u_{1}$ and $u=u_{2}$. The solution of Eq. (81) can be written in an implicit form as

$$
\frac{u_{2}}{\eta c_{0}} \xi=\int_{u_{2}}^{u} \frac{d u^{\prime}}{F\left(u^{\prime}\right)}+\text { const. }
$$

For this to describe a shock wave with a monotonic density profile, the function $F(u)$ must be positive at all internal points of the interval $\left(u_{2}, u_{1}\right)$. This criterion holds automatically in normal gasdynamics, but needs to be addressed in our case. It can be conveniently checked if we analyze graphically the relative position of the curve $P(1 / u)$ and the straight line $Y(u)$ passing through the points $\left(u_{1}, P_{1}\right)$ and $\left(u_{2}, P_{2}\right)$ :

$$
Y(u)=P_{2}-\frac{P_{2}-P_{1}}{u_{1}-u_{2}}\left(u-u_{2}\right) .
$$

Positiveness of the function $F(u)$ is achieved if the curve $P(1 / u)$ and the straight line $Y(u)$ do not intersect inside the interval $\left(u_{2}, u_{1}\right)$. For a given $u_{1}$, the maximum possible $u_{2}$ (that is, the minimum possible $\rho_{2}$ ) can be found from the condition of the straight line $Y(u)$ 's being an "upper" tangent to the curve $P(1 / u)$, as shown in line $b$ of Fig. 9.

Once the above conditions are met, integration in Eq. (82) gives a desired traveling-wave solution for any pre- 


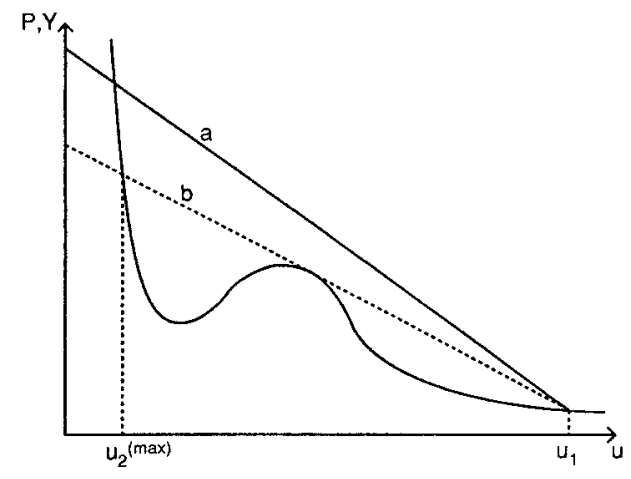

FIG. 9. Graphical analysis of the positiveness of the function $F(u)$ from Eq. (83), giving, for a fixed $u_{1}$, allowable values of $u_{2}$. The curve represents the graph of the effective pressure $P(1 / u)$, while the straight lines a and $\mathrm{b}$ represent the function $Y(u)$ from Eq. (83) for a typical allowable value of $u_{2}$ (a) and for the maximum allowable value of $u_{2}$ (b).

scribed function $P(\rho)$ and any admissible pair of $\rho_{1}$ and $\rho_{2}$ [see Meerson, Steele et al. (1993) for an example of such an integration]. However, similar to normal gasdynamics, two more conditions have to be checked in order that the anomalous shock wave be stable with respect to small perturbations in the direction of wave propagation. These are the "evolutionarity" conditions (Landau and Lifshitz, 1987) requiring the shock wave to move (i) supersonically relatively to the gas in front of the shock, and (ii) subsonically relatively to the gas behind the shock. Landau obtained these conditions from stability arguments only, comparing the number of independent parameters $n_{1}$ characterizing an arbitrary, small, initial perturbation of a shock to the number of equations $n_{2}$ relating these parameters. It appears that, only if the two evolutionarity conditions (i) and (ii) are satisfied, we have $n_{1}=n_{2}$ and the shock wave is longitudinally stable. It is important that these arguments do not invoke any thermodynamic considerations, and so they remain valid in our case of normal-anomalous gasdynamics. In our notation, the evolutionarity conditions can be written as a double inequality:

$$
\frac{\rho_{1}}{\rho_{2}}\left(\frac{d P}{d \rho_{1}}\right)^{1 / 2}<c_{0}<\left(\frac{d P}{d \rho_{2}}\right)^{1 / 2}
$$

where $d P / d \rho_{1,2}$ means $d P / d \rho$ evaluated at $\rho=\rho_{1,2}$.

Therefore, among the "elementary objects" of the normal-anomalous gasdynamics equations describing the long-wavelength limit of the radiative condensation, there are propagating shock waves and (standing) contact discontinuities. These elementary objects illustrate the gasdynamic bistability of the system, in contrast to the thermal bistability observed in the intermediatewavelength limit. In Sec. VI.C we shall discuss numerical solutions of the long-wavelength reduced equations and the role of these elementary objects in evolutionary problems.

\section{Numerical simulations}

For the numerical simulations, Meerson, Steele et al. (1993) adopted a cubic polynomial for $P(\rho)$ :

$$
P(\rho)=\frac{\rho^{3}}{9}-\frac{2}{3} \rho^{2}+\rho,
$$

so that

$$
\frac{d P}{d \rho}=\frac{1}{3}(\rho-1)(\rho-3) .
$$

The graph of $P(\rho)$ is shown in Fig. 2. The region of instability is $1<\rho<3$. The computational interval was $0<x<2 \pi$. Two types of sets of boundary conditions were used. In the first type, plasma inflow to the system (or outflow from the system) was allowed from both boundaries (an "open" system), and a fixed value of the density and a zero velocity gradient at each boundary were prescribed. In the second type of boundary condition, plasma inflow and outflow were not allowed, and the density gradient and plasma velocity were set to zero at each boundary (confined plasma).

Meerson, Steele et al. (1993) started with different initial conditions, mainly in the form of a small density or velocity perturbation around a uniform equilibrium $\rho=\rho_{0}$. Let us discuss here the case of a density perturbation, symmetric with respect to $x=\pi$,

$$
\rho(x, t=0)=\rho_{0}-\epsilon \cos (k x),
$$

and a zero initial velocity.

When the uniform equilibrium $\rho=\rho_{0}$ was stable [rising branches of the $P(\rho)$ curve], Meerson, Steele et al. (1993) observed propagation of acoustic waves and their damping by viscosity, as expected. For unstable equilibria [falling branches of the $P(\rho)$ curve], they observed localized instability, as predicted by the theory. Since at fixed $\rho_{0}$ and $\nu$ the linear growth rate (30) increases with wave number $k$, it is difficult to follow the evolution of modes with a small $k$, since narrow secondary peaks (emerging because of nonlinearities and numerical errors) become dominant. On the contrary, modes with sufficiently large $k$ can be conveniently studied as the linear growth rate for large $k$ approaches a plateau [see Eq. (30)], so that the secondary peaks do not interfere.

Meerson, Steele et al. (1993) followed the evolution of the mode $k=4$ in the case of a closed system for the initial conditions (87) with $\rho_{0}=1.2, \epsilon=0.001$, and different viscosities. As the effective pressure $P(\rho)$ is smaller in the region with increased density, inflow of the plasma into this region starts; so the density there keeps growing. Figure 10 shows the mode amplitude (plotted logarithmically) versus time for three different values of the viscosity. It can be seen that, after a short time (necessary for the system to develop a velocity perturbation consistent with the growing density perturbation), the mode growth enters the exponential stage predicted by the linear theory. The growth rates agree with those predicted by Eq. (30) with an accuracy of $2-3 \%$ for every value of $\eta$. Later, the evolution enters a nonlinear stage, when the mode amplitude first grows more quickly than in the linear stage (as predicted by anomalous gasdynamics), and then, after some oscillations, it becomes saturated. 


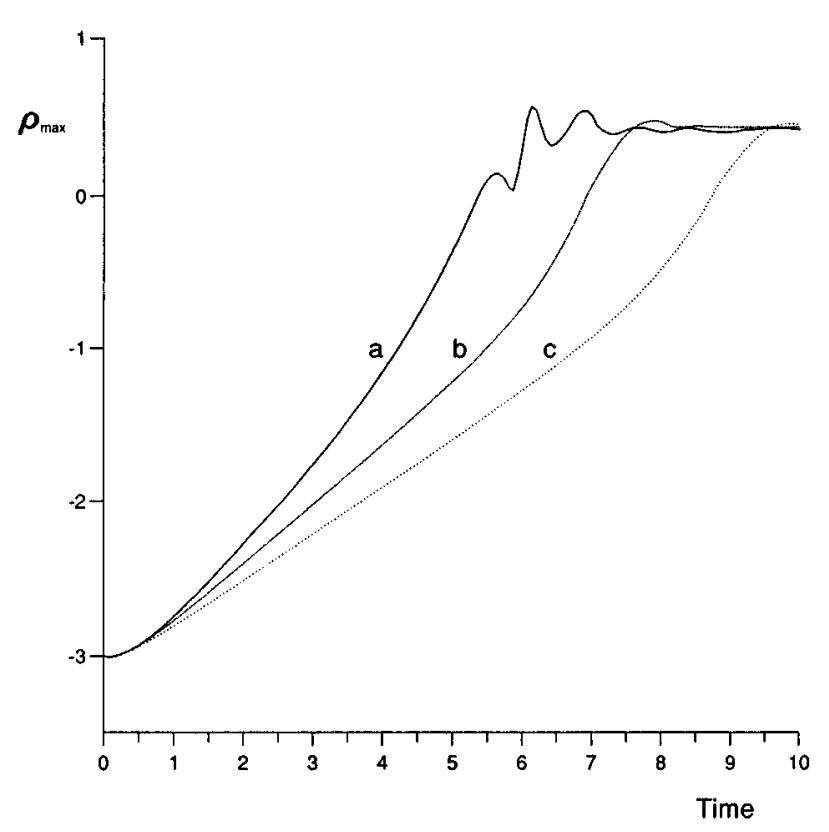

FIG. 10. Nonlinear evolution of the radiative condensation instability in the long-wavelength limit for the no-flux boundary conditions. Shown is the maximum density vs time for the initial condition (87) with $\rho_{0}=1.2, \epsilon=0.001$, and $k=4$. The viscosity $\eta=0.04$ (curve a), 0.1 (curve b), and 0.16 (curve c).

Figure 11 shows the evolution of the spatial profile of the same mode $k=4$ for $\eta=0.1$. It can be seen that stable nonuniform equilibrium condensations finally develop. As the plasma velocity vanishes at the final stage and the pressure becomes constant (not shown graphi-

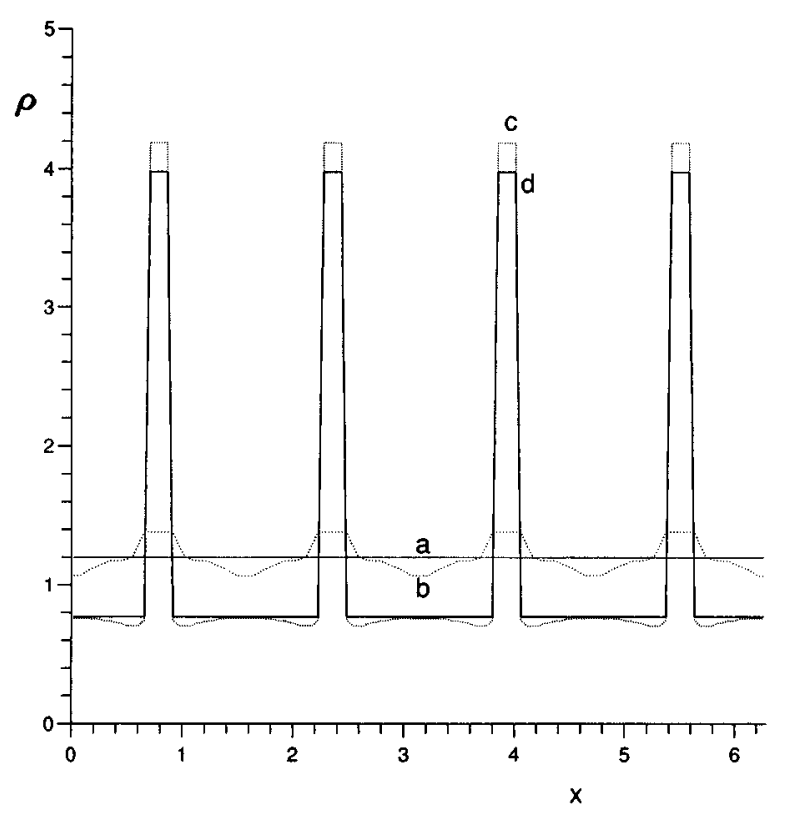

FIG. 11. Nonlinear evolution of the radiative condensation instability in the long-wavelength limit. Shown is the density profile vs time for the times $t=0$ (a), 6 (b), 8 (c), and 40 (d). The viscosity $\eta=0.1$, and other conditions are the same as in Fig. 10. cally), this result means that the plasma is cooler inside the condensations and hotter outside. The condensation boundaries clearly represent contact discontinuities, considered in Sec. VI.B. It is important to note that this stable equilibrium was made possible by disallowing any mass supply to the system. The process of formation of contact discontinuities remains intact for larger viscosities as well. In these cases, the condensation formation proceeds more slowly [as the linear growth rate (30) decreases], but residual velocities are damped more quickly.

In an open system, the initial stage of the instability looks similar. However, the later stage is quite different, as the forming radiative condensation starts to expand. The boundaries of the expanding radiative condensation represent two shock waves with very narrow fronts, traveling away from the center and separating stable higherand lower-density regions. Simultaneously, the material is evacuated from the lower-density periphery to the expanding condensation. The two shock waves represent anomalous shocks, discussed in Sec. VI.B in the context of traveling-wave solutions.

Meerson, Steele et al. (1993) performed special numerical experiments to study the shock-wave formation. They considered an open system and started with a discontinuity of the plasma density, $\rho=\rho_{l}$ at $x<x_{0}$, and $\rho=\rho_{r}$ at $x>x_{0}$, such that $\rho_{l}$ and $\rho_{r}$ were on the higherand lower-density stable branches of the $P(\rho)$ curve, respectively, while the initial velocity was zero. The higherdensity region $x<x_{0}$ was chosen to be at a higher pressure than the lower-density region, so that the discontinuity was expected to move into the lowerpressure region, that is, from left to right. Since the initial values of the densities (and zero velocities) on both sides of the discontinuity did not satisfy any particular traveling-wave solution, the discontinuity had to break into several discontinuities, propagating in both directions, before each of them approached a corresponding traveling-wave solution.

To save computation time, Meerson, Steele et al. (1993) started with a density discontinuity close to the left boundary. Therefore only those discontinuities that propagate from left to right were expected to settle down and form a traveling-wave solution. A typical case of such an experiment is shown in Fig. 12, where the densities $\rho_{l}$ and $\rho_{r}$ were chosen to be 4.9 and 0.5 (corresponding to pressures of 1.97 and 0.35 , respectively), $x_{0}$ was taken to be 5 , and the viscosity was $\eta=0.1$. This time a much longer spatial interval, $0<x<24 \pi$, was used to allow the system more time to evolve. Shown in Fig. 12 are the density profiles at different times. It is seen that, after some irregular transient stage, the discontinuity "adjusts" to a new value of the density on the left, $\rho_{2}=4.4$, and then propagates with almost a constant speed. To the right of the wave, the plasma remains static, while to the left, there is flow to the right, providing the plasma for the wave front to move. Equations (78) and (79) link the difference between the traveling- 


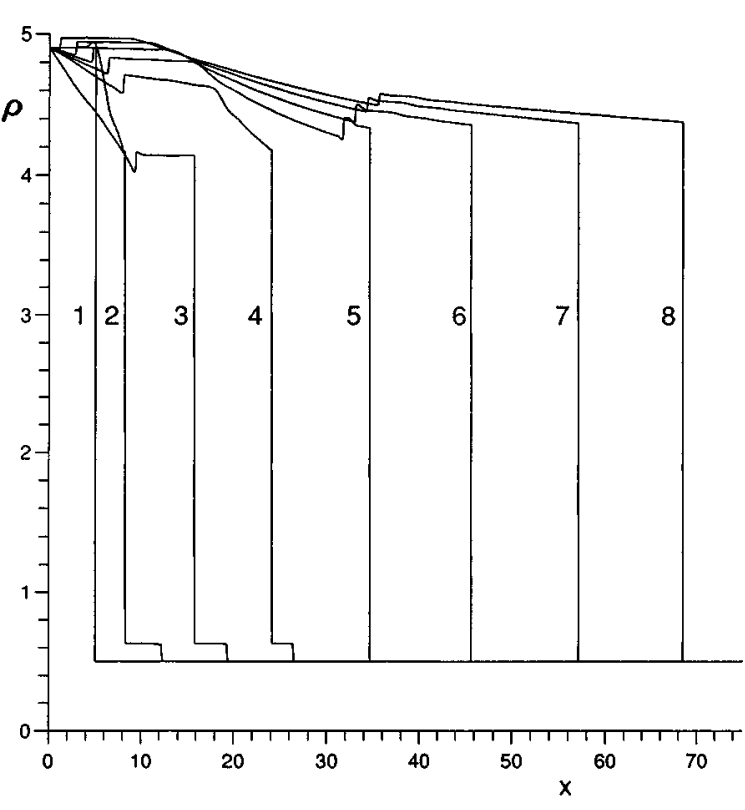

FIG. 12. Evolution of an arbitrary discontinuity of the density, as described by the long-wavelength equations for the radiative condensation instability. At the time moment $t=0$ (profile 1 ), a discontinuity is initiated with $\rho_{l}=4.9$ and $\rho_{r}=0.5$. At subsequent times $t=10,20,30, \ldots, 70$ (profiles 2-8), the propagating discontinuity is adjusting itself and evolving into an anomalous shock wave.

wave speed $c$ and the plasma velocities adjacent to the front, $v_{1}$ and $v_{2}$, with the densities and pressures to either side of the front.

Figure 13 shows separately the time evolution of the

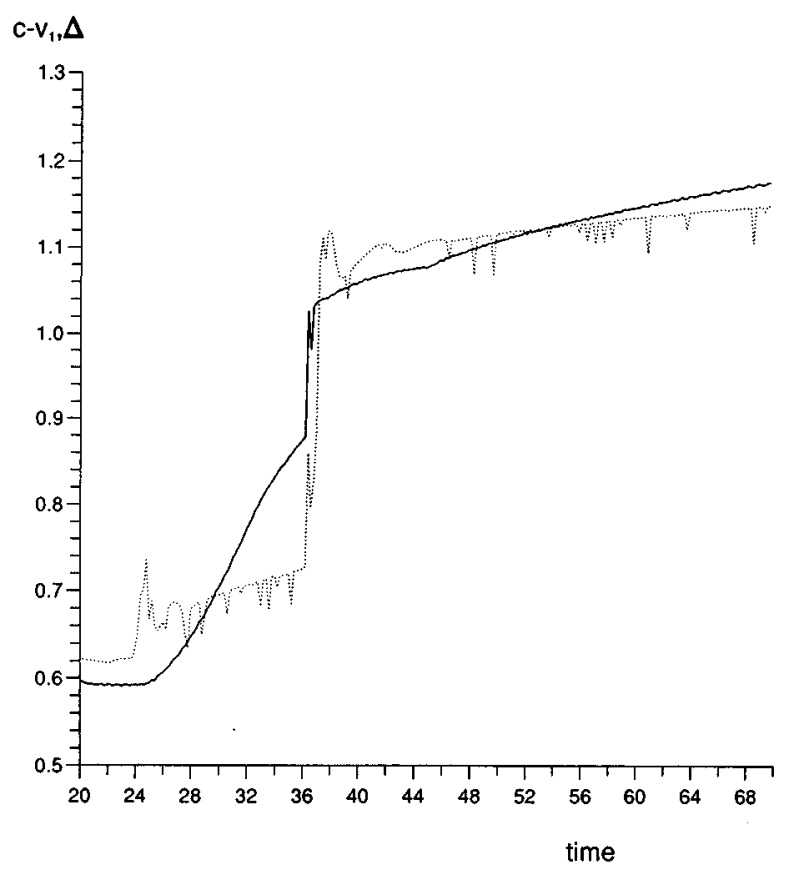

FIG. 13. Comparison of the shock-wave speed from Fig. 12 with its theoretical value. Shown in solid is the computed velocity difference $c-v_{1}$, while in broken is the function $\Delta$. square roots of the left- and right-hand sides of Eq. (79) in this run. Shown in solid is the computed velocity difference $c-v_{1}$, while in broken is the function

$$
\Delta=\left(\frac{P_{2}-P_{1}}{\rho_{2}-\rho_{1}} \frac{\rho_{2}}{\rho_{1}}\right)^{1 / 2} .
$$

It is seen that, starting from $t=36$, the two curves show a good agreement.

In addition, it was verified that the propagating shock wave finally developing in Fig. 12 satisfies the two evolutionarity conditions (84). It can also be seen directly from Fig. 12, where, starting from $t=40$, no perturbation is present on the right of the shock wave, while a small acoustic perturbation on the left, arising close to $t=40$, remains almost immobile, that is, moves to the left in the shock wave's reference frame.

Numerical integration in Eq. (77) makes it possible to find the steady-state density profile of the anomalous shock [Meerson, Steele et al. (1993)]. It appears that, on the higher-density side, the profile is much more abrupt than on the lower-density side. The same feature was observed in the time-dependent simulations of shockwave formation.

\section{Long-wavelength limit: summary and discussion of the planar case}

In the nonlinear stage of the long-wavelength radiative condensation instability, dense (and therefore cool) radiative condensations ("drops of liquid") are formed, surrounded by regions with a depleted density ("bubbles of vapor"). After some period of adjustment, accompanied by oscillatory plasma motions, the pressure in the condensations and in the surrounding lower-density regions becomes uniform. Further evolution of the system depends on the boundary conditions. In the case of a free plasma inflow (an open system), the radiative condensations expand, their boundaries representing the anomalous shock waves considered in Sec. VI.B. If the plasma inflow is not allowed (a closed or confined system), stable equilibrium condensations develop, the boundaries of which represent contact discontinuities.

It should be noted that both the contact discontinuities and the anomalous shock waves have been obtained in the framework of the long-wavelength reduced equations. Actually, these equations become invalid as steep density and temperature gradients develop in the process of instability and smaller spatial scales are introduced into the problem. Therefore the true structure of these "discontinuities" and shocks will not be determined by the small viscosity effects [employed in numerical simulations of Meerson, Steele et al. (1993) mainly to avoid wave breaking and to stabilize the numerical scheme]. Instead, one has to consider two additional physical factors. The first of them is related to the force balance setting in (for the small spatial scales that arise) on the same time scale as the thermal balance. The second factor is the heat conduction starting to act on the small spatial scales. 
In the limiting case of a relatively small heat conduction, $\lambda_{c} \ll \lambda_{a}$, and a long-wavelength initial perturbation, $k \ll \lambda_{a}^{-1}$, it is the first factor that dominates. The corresponding nonlinear theory is not available yet. In essence, such a theory should match a long-wavelength solution outside the fronts with an intermediatewavelength solution inside. One can assume that it is only the front width and structure that will be provided by a more complete theory, while all "macroscopic" results for anomalous shock waves (relations between $\rho_{1}, v_{1}$, and $P_{1}$ and $\rho_{2}, v_{2}$, and $P_{2}$ and evolutionarity conditions) will remain intact, as is the case for shock waves in normal gasdynamics.

Alternatively, in the case of a large heat conduction, $\lambda_{c} \ll \lambda_{a}$, one obtains the "direct crossover" regime, when thermal balance includes a significant heat flux. This regime was studied by Meerson, Steele et al. (1993). The results describe segregation in closed systems and uniformization in open systems. In the former case, the developing radiative condensation patterns are distinctly nonuniform.

The last question worth discussing here is that of the stability of the anomalous shock waves with respect to small transverse perturbations. Indeed, the onedimensional longitudinal stability arguments (leading to the evolutionarity conditions) are not sufficient, as the plane-wave front might suffer from corrugation instability in the transverse direction. General methods for the analysis of the transverse stability of shock waves in $a r$ bitrary gasdynamics were developed by Dyakov (1954). Employing Dyakov's criteria for the case of normalanomalous gasdynamics, Meerson and Rutkevich (1994) verified that the anomalous shock waves are stable with respect to the corrugation instability.

\section{RADIATIVE CONDENSATIONS IN TWO AND THREE DIMENSIONS}

Now we return to the intermediate-wavelength limit and extend the treatment to two- and three-dimensional geometries. We consider an isotropic plasma and assume that the pressure gradient is the only force acting on it. In two- and three-dimensional cases, the thermal conduction-controlled dynamics of radiative condensation in a bistable plasma becomes more complex, as two new factors, condensation/evaporation front curvature and possible transverse front instability, appear. The front curvature of large-scale patterns is small and therefore might seem insignificant. However, it proves to be quite important in the two following classes of problems. First, as the pressure of a confined plasma approaches the area-rule value (see Secs. V.B and V.C) and the front motions slow down, the curvature effects become dominant. We shall see that they determine both the possible final states of the system and the dynamics of relaxation towards the final states. Second, the front curvature represents an important stabilizing factor for the transverse instability of evaporation fronts, which can develop, as we shall see, both in isobaric and in confined plasmas.
Throughout this section we shall follow the recent extensive work by Aranson et al. (1995), who studied these problems in detail.

\section{A. Reduced equations}

When analyzing the intermediate- and shortwavelength radiative condensation in two- and threedimensional geometries, we cannot replace the momentum equation (2), wherein we neglect the viscosity, by the condition $\nabla p=0$, as this condition is not sufficient anymore for the determination of the velocity field. Therefore the reduction procedure should be done more carefully. In this section we outline two successive reductions of the governing equations (1)-(4). In the first reduction, one eliminates the acoustic modes. The second reduction (the corresponding equations will be called super-reduced) addresses plasmas that have already been radiatively segregated.

Similarly to the planar case, we shall be interested in two alternative regimes: isobaric condensations and condensations in a confined plasma. As we have seen in the planar case, the reduced equations for the isobaric case can be formally obtained from those of the confined plasma by going to the limit of an infinitely large system in the global pressure equation. It appears that this property holds in higher dimensions as well. Therefore we shall start with a confined plasma, and only in Sec. VII.F consider the isobaric regime.

Consider a plasma confined in some domain $\Omega$ (two or three dimensional), the maximal dimension of which is $L$. We denote $|\Omega|$ as the volume of the region $\Omega$ in the three-dimensional case or its area in the twodimensional case. Assume that the normal components of the plasma velocity and of the heat flux at the closed boundary $\Gamma$ of the domain $\Omega$ are zero:

$$
\begin{aligned}
& \left.\left(\mathbf{v} \cdot \mathbf{n}_{\Omega}\right)\right|_{\Gamma}=0, \\
& \left.\left(\nabla T \cdot \mathbf{n}_{\Omega}\right)\right|_{\Gamma}=0,
\end{aligned}
$$

where $\mathbf{n}_{\Omega}$ is the normal to the boundary $\Omega$. From Eqs. (1) and (88) immediately follows mass conservation:

$$
\int_{\Omega} \rho(\mathbf{r}, t) d \mathbf{r}=\text { const. }
$$

If the dimensions of the system are much less than the acoustic Field length, while the plasma motions are slow in comparison with the speed of sound $c_{s}$, we can simplify the original set of equations (1)-(4), so that the reduced system will not include the acoustic mode. For this purpose, let us represent the total plasma pressure $p(\mathbf{r}, t)$ as the sum of its spatially averaged part $P(t)$ and a spatially variable part $\tilde{p}(\mathbf{r}, t)$, so that

$$
p(\mathbf{r}, t)=P(t)+\tilde{p}(\mathbf{r}, t) .
$$

As the ratio $\tilde{p} / P \sim v^{2} / c_{s}^{2}$ is assumed to be small, we can neglect $\tilde{p}$ in Eqs. (3) and (4). Therefore the set of Eqs. (1)-(4) with the viscosity neglected can be rewritten as

$$
\frac{d \rho}{d t}+\rho \nabla \cdot \mathbf{v}=0
$$




$$
\begin{aligned}
& \rho \frac{d \mathbf{v}}{d t}+\nabla \tilde{p}=0, \\
& \frac{1}{\gamma-1} \frac{d P}{d t}+\frac{\gamma}{\gamma-1} P \nabla \cdot \mathbf{v}+\rho \mathscr{C}(\rho, T)-\nabla \cdot(K \nabla T)=0, \\
& P(t)=\frac{R}{\mu} \rho T,
\end{aligned}
$$

with the boundary conditions (88) and (89). In view of Eq. (95) we can also write

$$
\left.\left(\nabla \rho \cdot \mathbf{n}_{\Omega}\right)\right|_{\Gamma}=0 .
$$

Note that $\tilde{p}$ appears only in Eq. (93), and it can be eliminated completely if we apply curl to both sides of this equation. The resulting relation

$$
\nabla \times\left(\rho \frac{d \mathbf{v}}{d t}\right)=0
$$

replaces Eq. (93). In this sense, Eqs. (92)-(95) with the boundary conditions (88) and (89) represent a closed set. These are the reduced equations, applicable to the intermediate- and short-wavelength limits. The acoustic modes are eliminated from the equations. It can be checked that linear stability analysis of spatially uniform equilibria in the framework of the reduced set of Eqs. (92)-(95) immediately yields expression (7) for the linear growth rate.

Integrating Eq. (94) over the volume of the region $\Omega$ and using the boundary conditions, we obtain the following important relation:

$$
\dot{P}(t)=-\frac{\gamma-1}{|\Omega|} \int_{\Omega} \rho \mathscr{C}(\rho, T) d \mathbf{r},
$$

which determines the temporal evolution of the average plasma pressure. Equation (98) is similar to the "global pressure equation" of Begelman and McKee (1990), and it generalizes the one-dimensional equation (19).

Now we assume a powerlike thermal conduction, introduce scaled variables, as in the planar case (see Sec. III.A), and rewrite Eqs. (92)-(94) in the following scaled form:

$$
\begin{aligned}
& \frac{d u}{d t}=u \nabla \cdot \mathbf{v}, \\
& \frac{d \mathbf{v}}{d t}+u \nabla \tilde{p}=0, \\
& \frac{\dot{P}}{\gamma P}+\nabla \cdot \mathbf{v}+\lambda(u, P)-P^{\alpha} \nabla \cdot\left(u^{\alpha} \nabla u\right)=0
\end{aligned}
$$

with the boundary conditions (88) and

$$
\left.\left(\nabla u \cdot \mathbf{n}_{\Omega}\right)\right|_{\Gamma}=0 .
$$

The global pressure equation (98) can be rewritten as

$$
\frac{\dot{P}}{\gamma P}=-\frac{1}{|\Omega|} \int_{\Omega} \lambda(u, P) d \mathbf{r}
$$

and the carets are henceforth omitted.
In the isobaric regime, the corresponding reduced equations immediately follow from these by putting $|\Omega|=\infty$ in Eq. (103) and changing accordingly the boundary conditions.

\section{B. Large-scale equilibria}

Let us look for possible segregated, that is, spatially nonuniform, equilibria, $\mathbf{v}=\partial / \partial t=0$, of Eqs. (99)-(101), satisfying the boundary condition (102). These are described by the following equation:

$$
\nabla \cdot\left(u^{\alpha} \nabla u\right)=P_{\text {eq }}^{-\alpha} \lambda\left(u, P_{\text {eq }}\right),
$$

where $P_{\text {eq }}$ is the equilibrium pressure.

Notice that, if they exist, the equilibria described by Eq. (104) coincide with those of the original (unreduced) set of Eqs. (1)-(4). In addition, Eq. (104) holds in the isobaric regime, the difference being in the selection of the parameter $P_{\text {eq }}$ : in the isobaric regime, $P_{\text {eq }}$ is prescribed by the boundary conditions.

Equations similar to Eq. (104) appear in many contexts. For example, the same equation describes possible equilibria of a one-component, multidimensional, reaction-diffusion equation (Fife, 1979; Mikhailov, 1990):

$$
\frac{\partial u}{\partial t}=-P^{-\alpha} \lambda(u, P)+\nabla \cdot\left(u^{\alpha} \nabla u\right) .
$$

Finally, in the two-dimensional case, Eq. (104) with $\alpha=0$ describes a steady vortex flow of an ideal incompressible fluid (Lamb, 1932). In this case $u$ plays the role of a stream function, so that the vorticity is equal to $\nabla^{2} u$. Obviously, stability of the solutions to Eq. (104) in all above-mentioned problems depends on the specific time-dependent governing equations and is different in all these problems.

Although the one-dimensional solution of Eq. (104) is elementary (see Sec. V.B), not much is known about two- and three-dimensional analytic solutions, unless the function $\lambda(u, P)$ is a linear function of $u^{\alpha+1}$. A limited number of particular exact solutions can be found in the literature for specially selected nonlinear $\lambda(u, P)$ (Stuart, 1967; Batchelor, 1970; Shercliff, 1977; Kaptsov, 1988; Alfimov et al., 1990). Instead, we shall consider more general, though approximate, large-scale solutions that describe segregation of a bistable plasma into large regions occupied by the stable phases 1 (where $u=u_{1}$ ) and 2 (where $u=u_{2}$ ), with narrow transition layers between them. Therefore we shall concentrate on the bistable heating-cooling function $\lambda(u, P)$. For the large-scale segregated equilibria, both the right- and the left-hand sides of Eq. (104) are close to zero everywhere except in these transition layers. Therefore Eq. (104) is satisfied "trivially" outside the transition layers, while the "nontrivial" balance between the left- and right-hand sides of Eq. (104) determines the structure of the transition layers.

The simplest large-scale segregated equilibria are those with zero curvature, and they represent alternat- 
ing parallel plasma slabs with $u=u_{1}\left(P_{\text {eq }}\right)$ and $u=u_{2}\left(P_{\text {eq }}\right)$, with widths much larger than unity. In this case Eq. (104) becomes one dimensional, and we recover the planar solutions discussed in Sec. V.B. In this case, the equilibrium pressure $P_{\text {eq }}$ is equal (with an exponentially high accuracy) to the special value $P_{*}$, corresponding to the area rule.

The simplest solutions with a nonzero curvature represent a single cylindrical or spherical "drop" (phase 1) in the "vapor" (phase 2), or, alternatively, "bubble" (phase 2) in the "liquid" (phase 1). In these cases, Eq. (104) can be rewritten as

$$
\frac{d}{d r}\left(u^{\alpha} \frac{d u}{d r}\right)+\frac{d-1}{r} u^{\alpha} \frac{d u}{d r}=P_{\mathrm{eq}}^{-\alpha} \lambda\left(u, P_{\mathrm{eq}}\right),
$$

where $d=2$ for a circle (in two dimensions) or cylinder (in three dimensions), and 3 for a sphere. (This and most of the following relations containing $d$ are formally valid in the planar case $d=1$ as well.) We assume that the radius $R$ of any of these objects is much larger than the width of the transition layer between the phases (which is of the order of the conductive Field length, or unity in our scaled variables). As the radial derivative $d u / d r$ falls rapidly outside the transition layer, one can approximately replace the radial coordinate $r$ by $R$ in the denominator of the second term of Eq. (106). Now, for $R \gg 1$, the second term is much smaller than the first one, so that we can account for the curvature effects perturbatively. In the zero-order approximation, we return to the planar equilibrium problem. This yields the area rule (53), so that $P_{\mathrm{eq}}=P_{*}$, the area-rule value. Correspondingly, for the zero-order approximation of the function $u$, we have the relation (53) with $x$ replaced by $r$.

In the first-order approximation, we can replace the function $u$ in the second term of Eq. (106) by its zeroorder approximation and expand $\lambda(u, P)$ in the vicinity of $P=P_{*}$ :

$$
\lambda(u, P) \approx \lambda\left(u, P_{*}\right)+\mu(u)\left(P-P_{*}\right),
$$

where, as before, $\mu(u)=\partial \lambda(u, P) / \partial P$, evaluated at $P=P_{*}$, and $P-P_{*} \ll P_{*}$.

Now we multiply Eq. (106) by $u^{\alpha} d u / d r$ and integrate it from $r=0$ to the boundary $\Gamma$ of the plasma region. Then, using the fact that $d u / d r$ vanishes outside the transition layer and employing Eqs. (52) and (53), we arrive at the following relation:

$$
P_{\mathrm{eq}}=P_{*}+\frac{f}{g} \mathscr{K},
$$

where

$$
\begin{aligned}
& f=P_{*}^{\alpha} \int_{u_{1}}^{u_{2}} u^{\alpha}\left(\int_{u_{1}}^{u} \eta^{\alpha} \lambda\left(\eta, P_{*}\right) d \eta\right)^{1 / 2} d u / \int_{u_{1}}^{u_{2}}\left(\int_{u_{1}}^{u} \eta^{\alpha} \lambda\left(\eta, P_{*}\right) d \eta\right)^{1 / 2} d u \\
& g=P_{*}^{\alpha / 2} \int_{u_{1}}^{u_{2}} u^{\alpha} \mu(u) d u / \int_{u_{1}}^{u_{2}}\left(2 \int_{u_{1}}^{u} \eta^{\alpha} \lambda\left(\eta, P_{*}\right) d \eta\right)^{1 / 2} d u
\end{aligned}
$$

Therefore we have arrived at a linear relationship between the small correction $P_{\text {eq }}-P_{*}$ to the area-rule value $P_{*}$ and the small (dimensionless) curvature $\mathscr{K}$ of an equilibrium drop. The curvature $\mathscr{R}= \pm(d-1) / R$ is defined here to be positive for a drop and negative for a bubble. The $g$ factor (110) is the same as in the onedimensional theory [see Eq. (59)], while the $f$ factor is new. Notice that $f$ is always positive, which is important for the following analysis. For the simplest case of $\alpha=0$ (temperature-independent conductivity), $f=1$.

Equation (108) has been derived for "perfect" (cylindrical or spherical) drops and bubbles. However, its validity can be significantly extended to large nonspherical drops and bubbles with a smooth surface. In this case, the quantity $\mathscr{K}$, entering Eq. (108), is the sum of the two local principal curvatures (Gray, 1993) of the drop/ bubble surface.

In this new meaning, Eq. (108) has far-reaching consequences. Since the equilibrium pressure must be uniform, any equilibrium object must satisfy the condition of a constant mean curvature of its surface. [Notice a striking similarity between this problem and a classical problem of equilibrium of capillary surfaces without gravity (Finn, 1986). The similarity is quite unexpected, as no "conventional" surface tension is present in this problem.]

Most natural equilibrium patterns are those detached from the boundary $\Gamma$. In this case the constant mean curvature condition implies that only a circular (in two dimensions) or spherical (in three dimensions) drop or bubble (in addition to the zero-curvature, planar equilibria) can represent possible large-scale equilibrium patterns. The equilibrium pressure $P_{\text {eq }}$ is related to the radius of such a perfect radiative condensation according to Eq. (108). Furthermore, several circular or spherical drops/bubbles of the same radius, which are far apart, can represent possible equilibrium radiative condensations. It appears, however, that such many-drop (manybubble) equilibria are unstable (see below).

Therefore we have identified possible large-scale equilibrium radiative condensation in a confined plasma. Next we shall see that stability arguments introduce important additional limitations. 


\section{Higher-dimensional conductive relaxation and "super-reduced" equations}

In the relatively fast, radiative stage of the radiative condensation instability, the confined bistable plasma segregates into two locally stable phases. The following slower dynamics is determined by thermal conduction, and we call this stage conductive relaxation. Our immediate aim is to briefly describe the "super-reduced" equations that enable one to follow the relaxation of the system towards any of the equilibria, found in Sec. VII.B, if these equilibria are stable.

At the end of the radiative stage, the plasma density and temperature of each of the two phases becomes close to uniform outside the narrow transition layers, so that we describe these phases by the uniform values of their specific volume, $u_{1}(P)$ and $u_{2}(P)$ (therefore neglecting $\tilde{p}$ compared to $P$ everywhere except in $\nabla p)$. Following $P(t), u_{1}$ and $u_{2}$ can vary in time. Therefore, for the phase $i(i=1,2)$, the continuity equation (99) can be rewritten as

$$
\nabla \cdot \mathbf{v}_{i}=\frac{\partial \ln u_{i}(P)}{\partial P} \dot{P},
$$

while Eq. (100) takes the following form:

$$
\frac{d \mathbf{v}_{\mathbf{i}}}{d t}+u_{i}(P) \nabla \tilde{p}_{i}=0 .
$$

Taking curl of Eq. (112), we obtain

$$
\frac{\partial}{\partial t} \nabla \times \mathbf{v}_{i}=\nabla \times\left[\mathbf{v}_{i} \times\left(\nabla \times \mathbf{v}_{i}\right)\right] .
$$

At each interface, Eqs. (111)-(113) should be supplemented by matching conditions. In doing so, we treat the narrow transition layers as discontinuities. This implies, in particular, that the velocity field is assumed to be large scale, so that it has no components with wavelengths comparable to the conductive Field length.

As usual, the continuity and momentum equations result in the following matching conditions (Landau and Lifshitz, 1987):

$$
\begin{aligned}
& v_{i n}=c_{n}+j_{n} u_{i} \quad(i=1,2), \\
& \frac{v_{1 n}-c_{n}}{u_{1}} \mathbf{v}_{1 t}=\frac{v_{2 n}-c_{n}}{u_{2}} \mathbf{v}_{2 t}, \\
& u_{1}\left(\frac{v_{1 n}-c_{n}}{u_{1}}\right)^{2}+\tilde{p}_{1}=u_{2}\left(\frac{v_{1 n}-c_{n}}{u_{1}}\right)^{2}+\tilde{p}_{2} .
\end{aligned}
$$

Here $w_{n}=\mathbf{w} \cdot \mathbf{n}$ is the normal component of any vector $\mathbf{w}$ at each interface, and $\mathbf{w}_{\mathbf{t}}=\mathbf{w}-(\mathbf{w} \cdot \mathbf{n}) \mathbf{n}$. The normal vector $\mathbf{n}$ was defined after Eq. (57). $c_{n}$ is the normal component of the front velocity, and $j_{n}=\left(v_{i n}-c_{n}\right) / u_{i}$ is the flux of material through the interface.

In its turn, the thermal balance equation provides an expression for $j_{n}$. To obtain it, one should transfer to the reference frame, which moves with the velocity $c_{n}$, use the smallness of the transition layer width, and match the "inner" and "outer" solutions, as in the one- dimensional theory of Sec. V.B. For small $\mathscr{K}$ and $\left|P-P_{*}\right| \ll P_{*}$, one can show that

$$
j_{n}=-g\left(P-P_{*}\right)+f \mathscr{K}
$$

(Aranson et al., 1995).

Equations (88) and (102) remain the boundary conditions for the system. In the segregated plasma that we are considering now, Eq. (102) is satisfied automatically at the parts of the boundary $\Gamma$ embracing regions occupied by any of the "pure" phases 1 and 2 . If an interface meets the boundary $\Gamma$, the boundary conditions require each interface to be perpendicular to the boundary at their "meeting point."

Equations (111) and (112), combined with the matching conditions (114)-(117) at the interfaces and the boundary conditions, represent a closed set of "superreduced" equations and describe the conductive stage of the dynamics of large-scale radiative condensations in confined thermally bistable plasmas. It is also possible to find the corresponding super-reduced forms for the global pressure equation (103) and mass conservation:

$$
\begin{aligned}
\dot{P}= & \frac{P-P_{*}-\langle\mathscr{K}\rangle f / g}{\langle L\rangle} \\
& \times\left(\frac{g\left(u_{2}-u_{1}\right)^{2}\langle u\rangle}{\left(u_{2}-\langle u\rangle\right)\left(\partial u_{1} / \partial P\right)+\left(\langle u\rangle-u_{1}\right)\left(\partial u_{2} / \partial P\right)}\right)_{P=P_{*}} .
\end{aligned}
$$

This equation generalizes the corresponding planar result (63). The following notation has been used in Eq. (118):

$$
S=\iint_{\Omega_{I}} d S
$$

is the total area of interfaces $\Omega_{I}$;

$$
\langle L\rangle=\frac{|\Omega|}{S}
$$

is the characteristic size of segregated objects; and

$$
\langle\mathscr{K}\rangle=\frac{1}{S} \iint_{\Omega_{I}} \mathscr{K} d S .
$$

Finally,

$$
\langle u\rangle=\frac{|\Omega|}{\left|\Omega_{1}\right| / u_{1}(P)+\left|\Omega_{2}\right| / u_{2}(P)}=|\Omega|\left(\int_{\Omega} \frac{d \mathbf{r}}{u(\mathbf{r}, 0)}\right)^{-1}
$$

is the average specific volume. This quantity is defined by the first equality of Eq. (122). The second equality shows that $\langle u\rangle$ is independent of time, which implies that mass conservation holds in the super-reduced equations as it should.

Returning to the global pressure equation (118), we immediately see an important difference between the one-dimensional case $(\mathscr{K}=0)$ and the higherdimensional cases. The expression in the large parentheses of Eq. (118) is negative [see discussion after Eq. (64)]. Therefore, on the time scale $\tau_{1} \sim\langle L\rangle$, the pressure 
mismatch $P-P_{*}$ approaches $f\langle\mathscr{K}\rangle / g$ (which was zero in the one-dimensional theory). At the next (slower) stage, $P-P_{*}$ is approximately equal to $f\langle\mathscr{K}\rangle / g$. As the average curvature $\langle\mathscr{K}\rangle$ is (slowly) time dependent, $P-P_{*}$ follows $f\langle\mathscr{K}\rangle / g$ "adiabatically." The time-dependent quantity $\langle\mathscr{K}\rangle$, which does not appear in the onedimensional problem, introduces a new "degree of freedom" and therefore a new time scale $\tau_{2}$ (see later). This can result in qualitatively new effects, such as instability with respect to small changes in the sizes of drops and bubbles, Ostwald ripening type of dynamics, etc. Now we outline the linearized version of the super-reduced equations, which are convenient for the analysis of the linear stability of segregated equilibria. The linearization is performed near a static equilibrium, so that the unperturbed velocity is set to zero. The linearized continuity equation still looks like Eq. (111), except that the $P$ derivative in the right-hand side should be evaluated now at $P=P_{*}$. The linearized Euler equation (112) takes the form

$$
\frac{\partial \mathbf{v}_{\mathbf{i}}}{\partial t}+u_{i}\left(P_{*}\right) \nabla \tilde{p}_{i}=0
$$

while Eq. (113) yields

$$
\frac{\partial}{\partial t}\left(\nabla \times \mathbf{v}_{i}\right)=0 .
$$

This means that if the flow outside the transition layers is potential at $t=0$, it remains potential for all times. Furthermore, if the initial velocity is zero, we can introduce, instead of the pressure variation $\tilde{p}_{i}$, the following effective potential,

$$
\psi_{i}(\mathbf{r}, t)=-\int_{0}^{t} \tilde{p}_{i}\left(\mathbf{r}, t^{\prime}\right) d t^{\prime},
$$

and replace Eq. (123) by the following relation:

$$
\mathbf{v}_{\mathbf{i}}=u_{i}\left(P_{*}\right) \nabla \psi_{i}
$$

Linearization of the matching conditions (114)-(116) yields the following results. In Eq. (114) we evaluate $u_{i}$ at $P=P_{*}$ :

$$
v_{i n}=c_{n}+j_{n} u_{i}\left(P_{*}\right) .
$$

In the linear approximation, Eq. (115) is satisfied identically. Finally, in Eq. (116) the terms proportional to the square of the velocities can be neglected, so that it can be written as a continuity condition for the effective potential:

$$
\psi_{1}=\psi_{2} .
$$

Another way of linearizing the super-reduced equations becomes necessary when one studies the stability of a moving front, like that in the problem outlined in Sec. VII.F.

\section{Higher-dimensional conductive relaxation in confined plasmas}

Aranson et al. (1995) investigated several two- and three-dimensional stability problems for the equilibria found in Sec. VII.B. Here we shall briefly describe the results of these studies.

\section{Linear stability of a planar interface}

An arbitrary small perturbation of the equilibrium planar interface in a rectangular box can be represented as a volumetric perturbation with a zero wave number, accompanied by a pressure variation, and a set of isobaric deformation perturbations with nonzero wave numbers. All of these perturbations were shown to be damped independently. The volumetric perturbation relaxes on the time scale $\tau_{1} \sim L \gg 1$, predicted by the onedimensional theory. The deformation perturbations (more precisely, their lowest modes) decay much more slowly, on the time scale $\tau_{2} \sim L^{2} \gg \tau_{1}$.

\section{Shape stability of a perfect drop (bubble)}

Similarly, the linear stability of a perfectly circular or spherical drop of radius $R$ (phase 1), which is in equilibrium with an ambient "vapor" (phase 2), can be considered. In this case the equilibrium pressure $P_{\text {eq }}$ differs from the area-rule value $P_{*}$ by the correction $f /(g R)$, as predicted by Eq. (108). Alternatively, one can consider a bubble (region 2) surrounded by "liquid" (region 1). The simplest problem is formulated for a circular or spherical domain $\Omega$ concentric with the drop/bubble; however, the results remain valid for a general shape of the domain if $R \ll L$. Similarly to the previous problem, small volumetric (purely radial) perturbations of the drop/bubble, which are generally accompanied by a pressure variation, decouple from small (isobaric) deformations of the perfect shape of the drop/bubble interface. It appears that small deformations are damped with the characteristic time $\tau_{2} \sim R^{2}$.

\section{Radial stability of a perfect drop}

Now we consider the dynamics of purely radial perturbations of a single drop, which are accompanied by pressure variations. We shall use the (nonlinearized) superreduced equations in the limit of $P-P_{*} \ll P_{*}$ and will be able to go beyond the linear theory and consider large variations of the drop radius.

Consider a circular domain $\Omega$ containing a circular drop of radius $R$ concentric with $\Omega$ and surrounded by vapor (phase 2). Let $|\Omega|=S_{0}$ be the area of the domain $\Omega$. Let the drop radius and initial pressure be not in equilibrium, so that the system starts to evolve, $P$ and $R$ changing in time. Physically, mass and heat exchange between the drop and the vapor starts. Since the radial mode decouples from the (damped) azimuthal perturbations, $P(t)$ and $R(t)$ represent the only variables of the problem, and one needs two ordinary differential equations to describe the dynamics. The equation for $\dot{P}$ is provided by Eq. (118), where one just needs to calculate 


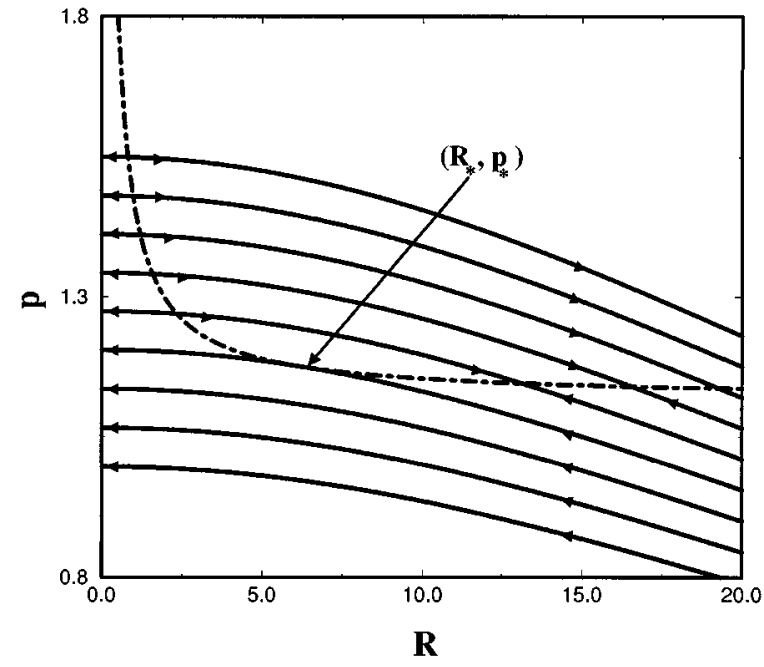

FIG. 14. Phase plane of Eqs. (130) and (131), describing the dynamics of radial perturbations of a single drop. The solid lines are the integral curves (132); the dashed line is the line of equilibria. The numerical values correspond to a specific choice of the heating-cooling function and parameters of the problem.

$\langle\mathscr{K}\rangle,\langle L\rangle$, and $\langle u\rangle$ using the two-dimensional versions of Eqs. (119)-(122). Then we make use of the mass conservation in the system,

$$
\frac{d}{d t}\left(\frac{\pi R^{2}}{u_{1}}+\frac{S_{0}-\pi R^{2}}{u_{2}}\right)=0 .
$$

After differentiation, one should linearize this relation around $P_{*}$. Finally, in the limit of a relatively small drop, $\pi R^{2^{*}} \ll S_{0}$, one obtains the following set of equations:

$$
\begin{aligned}
& \dot{P}=\frac{2 \pi u_{2}\left(u_{2}-u_{1}\right) R}{S_{0} \partial u_{2} / \partial P}\left[g\left(P-P_{*}\right)-\frac{f}{R}\right], \\
& \dot{R}=u_{1}\left[g\left(P-P_{*}\right)-\frac{f}{R}\right],
\end{aligned}
$$

where $u_{1,2}=u_{1,2}\left(P_{*}\right)$, and it has been assumed that neither $u_{1,2}$ nor their $P$ derivatives introduce large or small parameters.

The first integral of this system is

$$
P-\frac{\pi u_{2}\left(u_{2}-u_{1}\right) R^{2}}{S_{0} u_{1} \partial u_{2} / \partial P}=\text { const, }
$$

so that the integral curves on the phase plane $(R, P)$ represent descending quadratic parabolae (remember that $\left.\partial u_{1,2} / \partial P<0\right)$. The presence of this integral makes the problem solvable analytically. For any initial condition $R(0), P(0)$ the dynamics is determined by the relative position of the corresponding integral curve and the line of equilibria,

$$
g\left(P-P_{*}\right)-\frac{f}{R}=0 .
$$

Figure 14 shows the phase plane of the system. The solid lines are the integral curves (132), while the dashed line is the line of equilibria. For initial conditions lying on those integral curves, which do not intersect the line of equilibria, the drop will never reach an equilibrium. One can see that it will shrink and disappear in a finite time. (Actually, the theory breaks down for small drop radii. However, numerical simulations, free from this limitation, show that the drop evaporation indeed persists until the drop disappears.)

Now consider integral curves, which have two intersection points with the line of equilibria. These points represent two equilibria, the first with a smaller and the second with a larger drop radius. The smaller-radius equilibrium proves to be unstable, so that the drop either shrinks and disappears, or expands until a new equilibrium with a larger drop radius is reached.

Therefore the line of equilibria consists of two parts, unstable and stable. The stability border, dividing the line of equilibria into these two parts, is the tangency point between the line of equilibria and an integral curve. This point can be easily found, and it gives the minimum value $R_{*}$ of the stable drop radius:

$$
R_{*}=\left[-\frac{f u_{1} S_{0} \partial u_{2} / \partial P}{2 \pi g u_{2}\left(u_{2}-u_{1}\right)}\right]^{1 / 3} .
$$

We see that, while the minimum area of a stable drop, $\pi R_{*}^{2}$, increases with $S_{0}$ like $S_{0}^{2 / 3}$, its relative value $\pi R_{*}^{2} / S_{0}$ decreases like $S_{0}^{-1 / 3}$. Equation (133) can also be obtained from the marginality condition of a linear stability analysis of Eqs. (130) and (131).

Three-dimensional problems of the radial stability of a single drop can be analyzed similarly. In summary, an individual equilibrium drop (bubble) can be either stable or unstable with respect to a pure radial mode. An unstable drop (bubble) either shrinks and disappears in a finite time, or expands until a stable equilibrium is reached.

\section{Nonlocal interaction between drops: Ostwald ripening}

Now let us consider interaction between $N \geqslant 2$ drops or bubbles. The radii of the drops are assumed to be large enough, $R_{i} \gg 1, i=1,2, \ldots, N$, and the distances between the drops and the boundary, and between the drops themselves, are much larger than the drop radii. In this small-volume-fraction limit, the drop-drop interaction results solely from the (spatially uniform) pressure variations in time. This interaction causes radial variations of the drops, while their shape deformations remain small. As we have already seen, the interaction vanishes in equilibrium, when all the drops have the same radius. However, this equilibrium is unstable (see below). Therefore our aim is to describe the dynamics of this system and its relaxation to the final state.

Using Eq. (117) for each of the drops, and the total mass conservation in the (three-dimensional) system, Aranson et al. (1995) arrived at the following set of equations:

$$
\frac{d \Delta p}{d \tau}=-\epsilon\left(\Delta p \sum_{i=1}^{N} R_{i}^{2}-\sum_{i=1}^{N} R_{i}\right),
$$




$$
\frac{d R_{i}}{d \tau}=\Delta p-\frac{1}{R_{i}}, \quad i=1,2, \ldots, N,
$$

the first integral of which is

$$
\Delta p+\frac{\epsilon}{3} \sum_{i=1}^{N} R_{i}^{3}=\text { const. }
$$

Here a scaled time $\tau=2 u_{1} f t$ and pressure

$$
\Delta p=\frac{g\left(P-P_{*}\right)}{2 f}
$$

have been introduced. Furthermore,

$$
\epsilon=-\frac{2 \pi g u_{2}\left(u_{2}-u_{1}\right)}{f V_{0} u_{1} \frac{\partial u_{2}}{\partial P}}>0 .
$$

Equations (134) and (135) have simple equilibria $R_{i}=R_{0}$ (that is, $N$ identical drops) and $\Delta p=1 / R_{0}$, which can be easily shown to be unstable with respect to small variations of the drop radii. Indeed, a small difference in any two drops' radii grows exponentially in time, the characteristic growth time (in the "old" units of time) being $R_{0}^{2}$. The monotonic growth of the difference in the radii of any two drops in time continues far from the equilibrium. As a consequence, the initial ordering of the drop radii (let it be $R_{1}<R_{2}<\cdots<R_{N}$ ) persists. On the other hand, the total volume occupied by the drops is always finite. It follows that at least one drop must be shrinking, and it is the smallest drop whose radius first reaches zero. This singularity develops in a finite time, so that the smallest drop disappears, and the number of drops becomes $N-1$. At this stage (after the singularity), we have to redefine the "initial conditions" and the first integral, then employ the same arguments, and so on.

Therefore the dynamics of an ensemble of drops proceeds as a "cruel" competition mediated by the timedependent uniform pressure of the system. The smallest drop shrinks and disappears first. Then goes the second smallest drop, etc. The process continues until only one drop (the largest) remains. The same result is valid for an ensemble of $N$ bubbles. This type of dynamics, when larger drops thrive at the expense of smaller ones, is known in the dynamics of the first-order phase transitions as Ostwald ripening (Ostwald, 1900).

\section{Many drops' statistics and universal scalings}

Now let us assume that a large number of drops, $N \gg 1$, has developed in the earlier conductive stage. Introduce the (time-dependent) distribution function of the drops with respect to their radii, $F(R, \tau)$. By definition, $\int_{0}^{\infty} F(R, \tau) d R=n(\tau)$, where $n(\tau)$, the number of drops per unit volume (drop concentration), is a continuous function of the scaled time $\tau$.

In the limit of a small volume fraction, the distribution function must satisfy the continuity equation in the space of radii,

$$
\frac{\partial F}{\partial \tau}+\frac{\partial}{\partial R}(\dot{R} F)=0, \dot{R}=\Delta p-\frac{1}{R} .
$$

The first integral (136) can be rewritten as

$$
\Delta p+\frac{\epsilon_{0}}{3} \int_{0}^{\infty} R^{3} F(R, \tau) d R=Q=\text { const }
$$

where

$$
\epsilon_{0}=-\frac{2 \pi g u_{2}\left(u_{2}-u_{1}\right)}{f u_{1} \partial u_{2} / \partial P}>0
$$

Equations (137) and (138) represent a closed set. Given the initial conditions $F(R, 0)$ and $\Delta p(0)$, they determine the whole evolution of the system up to the time when the number of drops becomes small, and the statistic description fails.

In this formulation the problem becomes very similar to the classical problem of the kinetics of diffusive decomposition of supersaturated solid solutions, studied in the pioneering work by Lifshitz and Slezov (1958). The main difference lies in the expression for $\dot{R}$, which in their case included an additional factor $1 / R$. The meanfield approach of Lifshitz and Slezov was adopted in many other systems, showing Ostwald ripening [for a recent bibliography on the Ostwald ripening theory, including an account of finite-volume fraction effects, see Slezov and Sagalovich (1987), Marder (1987), Brown (1992), Chen and Voorhees (1993), and Yao et al. (1993)]. Among them, Wagner (1961) was the first to consider a diffusively decomposing system with a chemical reaction on the interphase surface. Surprisingly, Eqs. (137) and (138) exactly coincide with the corresponding (scaled) equations of Wagner.

The theory of Lifshitz and Slezov predicts that, for sufficiently large times, any extended (that is, positive on the whole interval $0<R<\infty$ ) distribution function of the drops with respect to their radii $R$ approaches a universal self-similar form, which represents an intermediate asymptotics of the problem. [For an authoritative reference on self-similarity and intermediate asymptotics, see the book by Barenblatt (1979).] For the system (137) and (138), the general form for the self-similar distribution function is $F(R, \tau)=\tau^{-2} \Phi\left(R / \tau^{1 / 2}\right)$. Correspondingly, the scaled pressure mismatch $\Delta p$ decreases like $\beta \tau^{-1 / 2}$. This implies that the "drop concentration" $n$ decreases like $\eta \tau^{-3 / 2}$, while the average drop radius grows like $\chi \tau^{1 / 2}$. The positive coefficients $\eta, \chi$, and $\beta$ are uniquely determined by the zero, first, and second moments of the function $\Phi(\xi)$. The function $\Phi$ satisfies an ordinary differential equation of the first order and a normalization condition, which one obtains from Eqs. (137) and (138). This ordinary differential equation can be integrated by separation of variables, and we arrive at the following expression:

$$
\begin{aligned}
\Phi(\xi)= & \text { const } \cdot \xi\left|\xi^{2}-2 \beta \xi+2\right|^{-5 / 2} \\
& \times \exp \left(-3 \beta \int \frac{d \xi}{\xi^{2}-2 \beta \xi+2}\right) .
\end{aligned}
$$


Further calculations depend on $\beta$. It can be checked that the case of $0<\beta<\sqrt{2}$ must be ruled out as nonnormalizable.

For $\beta=\sqrt{2}$, we arrive at the solution found by Wagner (1961):

$$
\Phi(\xi)=\text { const } \cdot \xi(\sqrt{2}-\xi)^{-5} \exp \left(-\frac{3 \sqrt{2}}{\sqrt{2}-\xi}\right)
$$

for $\xi<\sqrt{2}$ and $\Phi(\xi)=0$ elsewhere. The constant is determined by the normalization condition. This solution vanishes at $\xi=\sqrt{2}$ together with all its derivatives. For the Wagner solution, $\chi=(8 / 9) \sqrt{2}$. Extending the arguments of Lifshitz and Slezov (1958) and Slezov (1978) to this case, one can see that this particular solution is the attractor of any extended initial distribution.

In the case of $\beta>\sqrt{2}$, formal integration in Eq. (139) yields

$$
\begin{aligned}
\Phi(\xi)= & \text { const } \cdot \xi\left|\xi_{-}-\xi\right|^{-5 / 2+3 \beta /\left(2 \sqrt{\beta^{2}-2}\right)} \\
& \times\left|\xi_{+}-\xi\right|^{-5 / 2-3 \beta /\left(2 \sqrt{\beta^{2}-2}\right)}
\end{aligned}
$$

where $\xi_{ \pm}=\beta \pm \sqrt{\beta^{2}-2}$. Aranson et al. (1995) constructed a positive, nonsingular solution, using Eq. (141) on the interval $\left(0, \xi_{-}\right)$and setting $\Phi(\xi)=0$ for $\xi \geqslant \xi_{-}$. The condition $\Phi\left(\xi_{-}\right)=0$ requires that $\beta<5 \sqrt{2} / 4$ (otherwise, $\Phi$ diverges at $\left.\xi=\xi_{-}\right)$.

Therefore there is a one-parameter family of selfsimilar distribution functions defined on a quite narrow interval of the parameter $\beta: \sqrt{2} \leqslant \beta<5 \sqrt{2} / 4$, the left boundary of which corresponds to the Wagner solution. [Similar localized distributions can be constructed in the problem of Lifshitz and Slezov as well, as was shown numerically by Brown $(1989,1990)$.] Notice that the exponents of the power laws of the pressure mismatch, drop concentration, and average drop radius as functions of time are independent of $\beta$. However, the coefficients $\beta, \eta$, and $\chi$ in the corresponding power laws (and, of course, the shape of the distribution function) vary with $\beta$.

An important question is which of these self-similar distributions will actually develop, if one starts from a prescribed initial distribution. As mentioned above, it is the Wagner solution (140) that finally sets in for an extended initial condition. The case of a localized initial condition requires an additional analysis. [A similar question of the realizability of different self-similar solutions has arisen for the Lifshitz-Slezov case, where it has been an object of much controversy (Brown, 1992; Chen and Voorhees, 1993).] Essentially, this problem concerns finding the selection rule for the "correct" self-similar distribution function of the drops. This problem was addressed quite recently for the model (137), (138) by Meerson and Sasorov (1996). They considered a localized initial distribution, which is positive on the interval $\left(0, R_{m}(t=0)\right)$ and zero for $R>R_{m}(t=0)$. They showed that, first, the solution always remains localized on an interval $\left(0, R_{m}(t)\right)$. Furthermore, the leading term of the expansion of $F(R, t)$ in the vicinity of $R=R_{m}(t)$ can be written as $A(t)\left[R_{m}(t)-R\right]^{v}$, where $v$ is uniquely determined by the initial condition:

$$
v=\left.\frac{d \ln F(R, t=0)}{d \ln \left[R_{m}(t=0)-R\right]}\right|_{R=R_{m}(t=0)} .
$$

It is crucial that parameter $v$ remains invariant for any localized solution of the problem. Therefore it is the double-logarithmic derivative (142) of the initial distribution, evaluated at $R=R_{m}$, that selects the correct self-similar distribution function from Eq. (141).

It should be remembered that, no matter what the precise form of the "intermediate" self-similar solution is, the final state of the dynamics must be a single drop (bubble).

\section{E. Numerical simulations}

Aranson et al. (1995) carried out two-dimensional numerical simulations aimed at verifying their superreduced conductive relaxation model and some of the analytical results, discussed previously in Sec. VII. They worked with the reduced model, described by Eqs. (99), (101), and (103) and the boundary conditions (88) and (102). However, instead of the Euler equation (100), they employed a much simpler equation for the velocity field:

$$
\mathbf{v}=u \nabla \psi .
$$

As we have seen, this relation becomes accurate close to equilibrium $P=P_{*}$ [see Eq. (126)].

The equations were solved in a square domain $L \times L$ with the no-flux boundary conditions. $L$ was varied from 35 to 60 , so that it was always much larger than the conductive Field length (that is, unity in the scaled variables). The same bistable heating-cooling function (49) as that used in the planar calculations was employed, and $\gamma=5 / 3$ and $\alpha=0$ were chosen. In this case, the arearule value of the pressure is $P_{*}=\sqrt{5} / 2 \simeq 1.118$, while $g=2 \sqrt{2} \simeq 2.828$ and $f=1$.

The first series of simulations concerned the dynamics of a system of two or more drops with different radii. Figures 15 and 16 show the two-drop dynamics, obtained numerically. (In these simulations, periodic boundary conditions with a period $L=60$ were employed.) It is seen from Figs. 15(a)-15(c) that the smaller drop shrinks and disappears as predicted, with the larger drop and vapor thriving at its expense. Figure 16 shows the corresponding pressure history. Calculating the equilibrium radius $R_{\text {eq }}$ from the relation

$$
g\left(P-P_{*}\right)-\frac{f}{R}=0
$$

(with $P \simeq 1.15$ as in Fig. 16), we obtain $R_{\text {eq }} \simeq 11$, which is quite close to the visible radius of the drop in Fig. 15(c). Noticeable in Fig. 15 is an almost ideal preservation of the circular forms of the drops, which justifies the assumption of a purely radial interaction of the drops. When starting with three drops of different radii, one observes shrinkage and disappearance of the smallest 


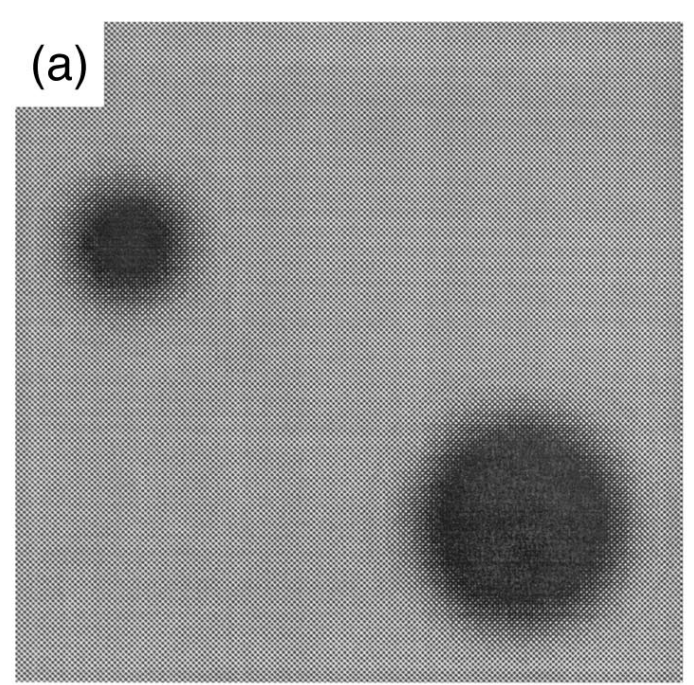

\section{(b)}

\section{(c)}

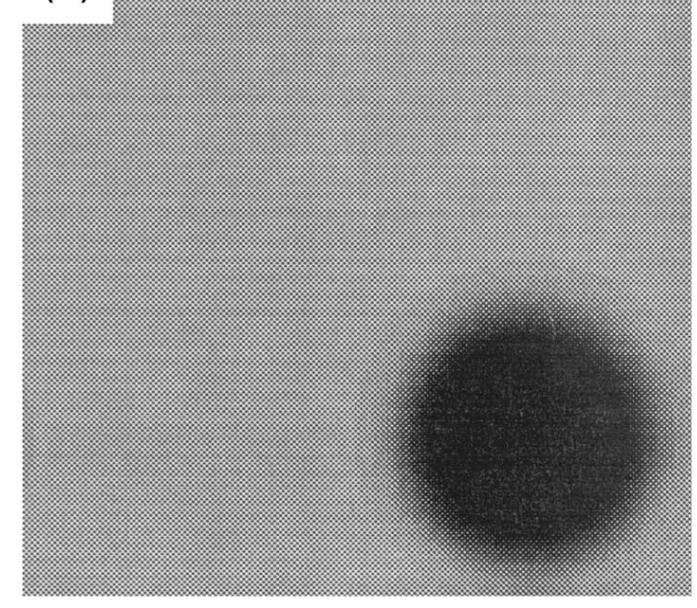

FIG. 15. Background-mediated competition between two drops, studied numerically. The initial condition represents two drops $\left(u=u_{1}\right)$ in the "vapor" $\left(u=u_{2}\right)$; the initial pressure is 1.2. The dimensions of the system are $60 \times 60$. Shown are the specific-volume contours at $t=5$ (a), 45 (b), and 100 (c).

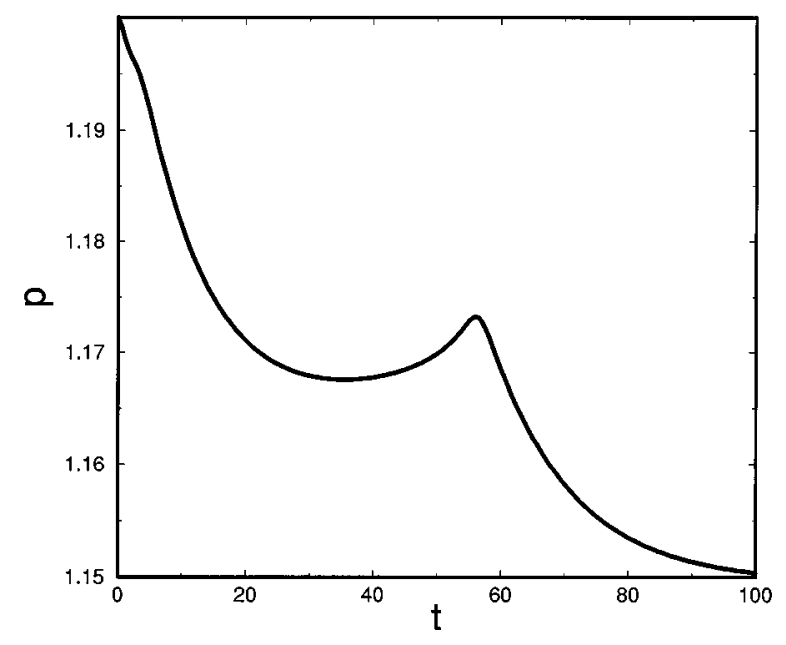

FIG. 16. Pressure history for the same run as in Fig. 15. The peak at $t \simeq 57$ corresponds to the moment of the disappearance of the smaller drop. The pressure finally approaches 1.15, which agrees with the surviving drop's radius in Fig. 15(c).

one, followed by shrinkage of the next smallest. Therefore "full" numerical simulations confirm the theoretical predictions concerning the Ostwald ripening type of competition between the drops.

In the next series of runs (Figs. 18-21), the initial condition for $u$ represented broadband "noise": a smallamplitude multimode perturbation $\delta u(x, y)$ around a uniform state $u_{0}$. For radiative segregation to develop, the value of $u_{0}$ must be somewhere between $u_{1}$ and $u_{2}$, and not too close to any of them. In the beginning (stages 1 to 3 of the dynamics), the simulation results always look similar to the planar case. First, the pressure drops to $u_{0}$, so that $u_{0}$ quickly becomes an unstable equilibrium (stage 1 ). Then the linear stage of the radiative condensation instability develops (stage 2) when the pressure is almost constant. Perturbations with too short wavelengths are strongly damped [see Eq. (7)], and they are already absent after a few units of time. In stage 3, strong plasma segregation develops: higher-density, cooler regions are formed (phase 1), which are surrounded by lower-density, hotter regions (phase 2).

The subsequent evolution of the forming patterns was observed to proceed in two different ways, depending on the initial conditions. In Figs. 17 and 18, where $u_{0}=0.9$, straightening of the interface between the phases 1 and 2 occurs, and a slab-type equilibrium develops. Figure 18 shows that the pressure approaches $P_{*} \simeq 1.118$, in full agreement with the theory. In addition, the final stage of this straightening is described by the linearized theory, as predicted. Indeed, in Fig. 17(c), one can still see a slightly deformed interface, and the deformation mode is the fundamental, $n=1$. By this time the shorter-wavelength modes, $n=2,3, \ldots$, have already disappeared, as their damping rates are $4,9, \ldots$ times higher.

On the contrary, Figs. 19 and $20\left(u_{0}=1.4\right)$ show the process of formation of a single equilibrium circular drop. Actually, Fig. 19(c) shows a quarter of a drop, a 

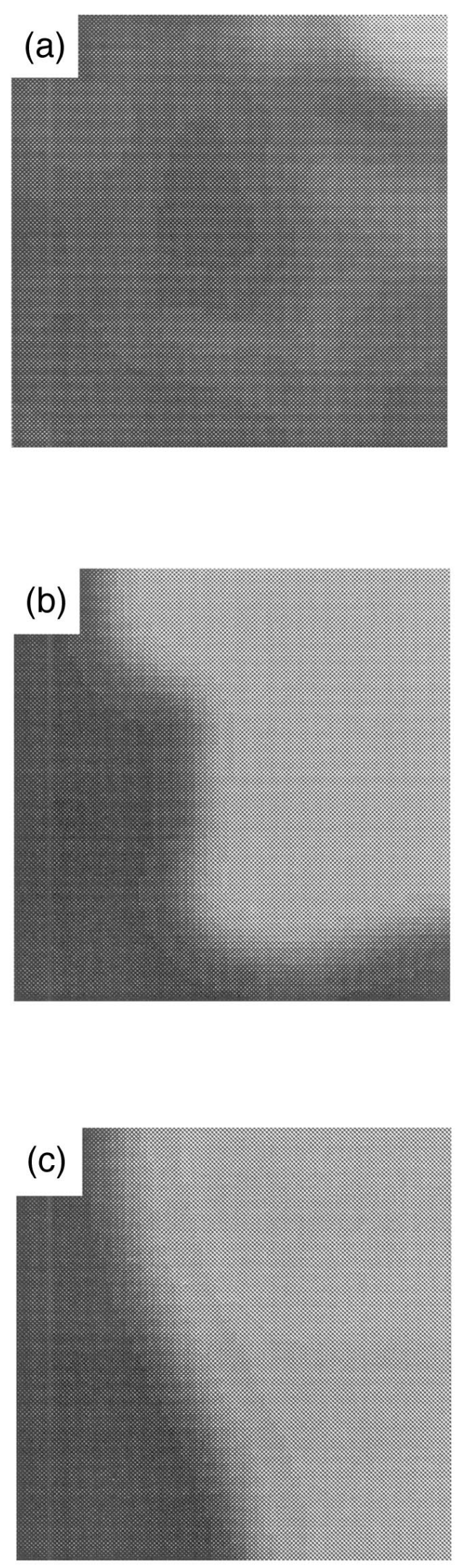

FIG. 17. Slab-type equilibrium developing from broadband noise. The initial condition is a small-amplitude multimode perturbation of $u$ around $u_{0}=0.9$. The initial pressure is 1.0; the dimensions of the system are $40 \times 40$. Shown are the specific-volume contours at $t=10$ (a), 20 (b), and 250 (c).

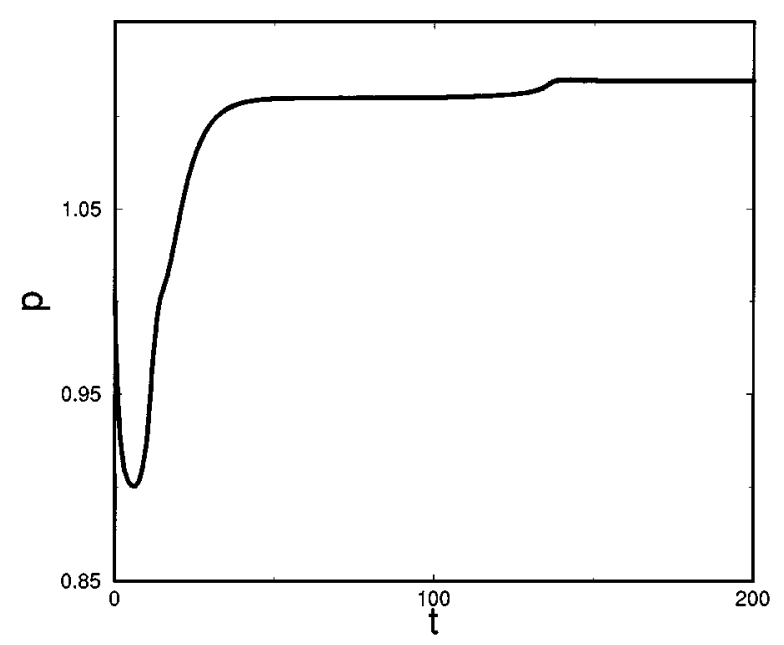

FIG. 18. Pressure history for the same run as in Fig. 17. The pressure first drops to 0.9 (so that the radiative condensation instability starts to develop) and finally approaches the arearule value $P_{*}=1.118$.

compromise between the drop-type equilibrium and an "attached" equilibrium, obviously possible in the square domain. The circular form of the forming drop supports theoretical predictions of the drop stability with respect to small azimuthal perturbations. The final (equilibrium) pressure (Fig. 20) agrees with the drop radius and is predicted quite accurately from initial conditions.

Therefore Aranson et al. (1995) were able to reproduce numerically both types of large-scale equilibria predicted by their theory, verify the stability analyses based on the super-reduced equations, and observe the background-mediated competition between drops (Ostwald ripening). Direct numerical experiments with a large number of drops, aimed at investigating the statistical properties of Ostwald ripening, have not been performed yet.

\section{F. Transverse instability of evaporation fronts}

We shall discuss here the three-dimensional intermediate-wavelength dynamics of radiative condensation in plasmas with isobaric boundary conditions. We already know that the corresponding reduced model is based upon elimination of the acoustic modes. Consider a bistable plasma. The initial stages of the dynamics are not sensitive to the boundary conditions. Indeed, during the radiative stage of the radiative condensation instability, the plasma is segregated into two phases. In the conductive stage, which is next, thermal conduction becomes important. One of the phases starts to expand, while the other shrinks, as predicted by the planar theory reviewed in Sec. IV. Depending on the sign of the integral $I$ [see Eq. (42)], either condensation or evaporation occurs. The planar theory predicts that, as the result of this condensation (evaporation) process, it is the dense and cool (dilute and hot) phase that will finally survive. As the plasma pressure is constant and 

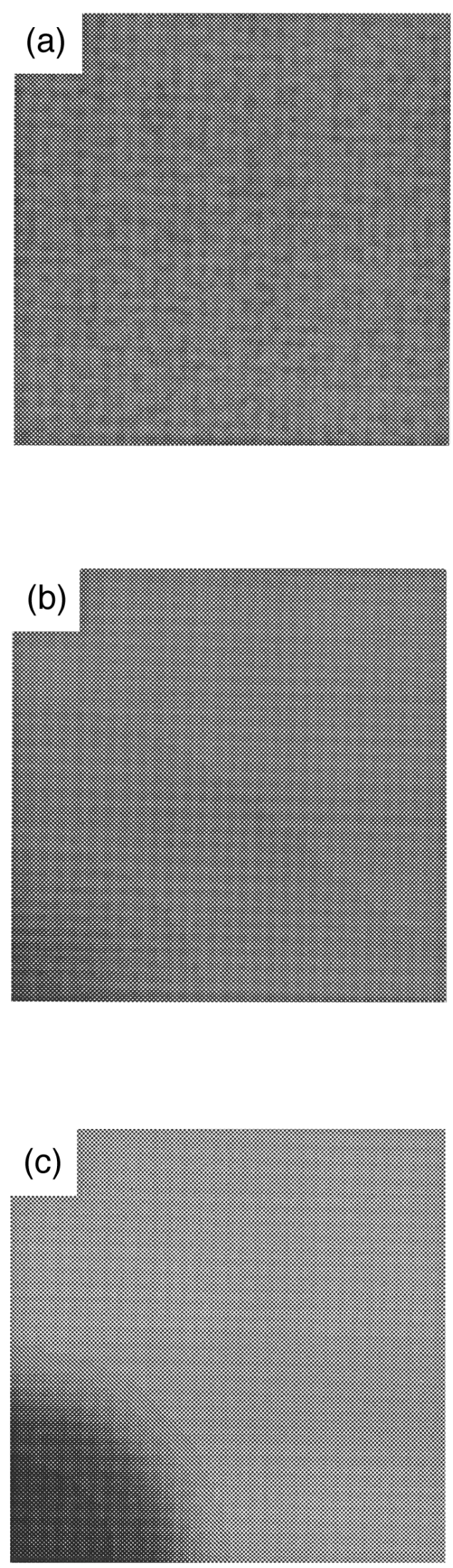

FIG. 19. Drop-type equilibrium developing from broadband noise. The initial condition is a small-amplitude multimode perturbation of $u$ around $u_{0}=1.4$. The initial pressure is 1.2; the dimensions of the system are $35 \times 35$. Shown are the specific-volume contours at $t=0$ (a), 20 (b), and 180 (c).

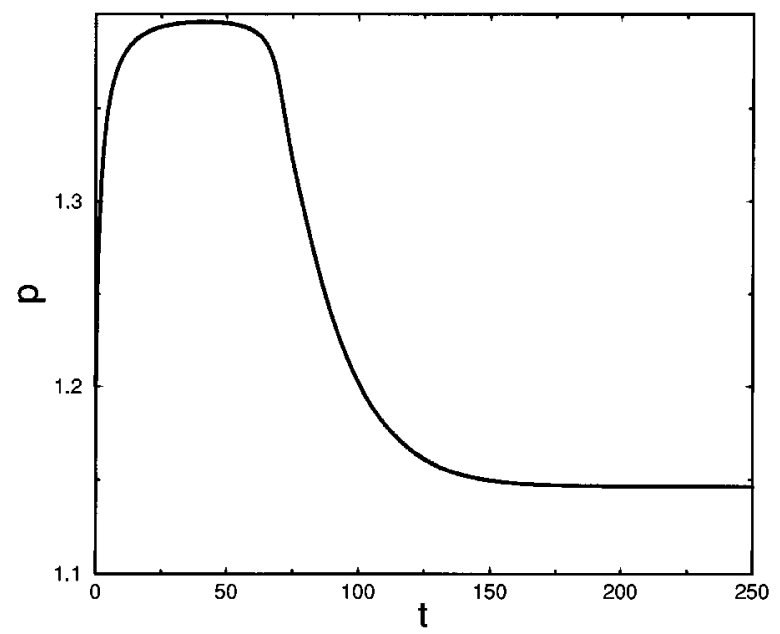

FIG. 20. Pressure history for the same run as in Fig. 19. The pressure first jumps close to 1.4 (so that the radiative condensation instability starts to develop) and finally approaches the value corresponding to the equilibrium radius of the forming drop.

plasma inflow or outflow is allowed, there is no mechanism that would arrest the front motions and preserve the segregation. However, the planar front motion can become unstable with respect to transverse perturbations, and we need a three-dimensional theory to investigate this problem. In the following we briefly review the transverse stability theory for the moving condensation or evaporation fronts.

As we have already seen, the isobaric version of the reduced equations can be formally obtained from the equations, derived for confined plasmas, by letting the size of the "box" go to infinity in the global pressure equation (98). In addition, one can assume for simplicity that the pressure $P=$ const is close to the area-rule value $P_{*}: \Delta P=\left|P-P_{*}\right| \ll P_{*}$. In this case we can use the simple linear relation (117) between the flux of matter $j_{n}$ through the "discontinuity," the pressure mismatch $\Delta P$, and the front curvature $\mathscr{K}$.

Let us consider an unperturbed planar front, which is located at the plane $x=0$ of a Cartesian coordinate system, moving together with the front. A half-space $x>0$, indexed by $D$, is assumed to be downstream; and a half-space $x<0$, indexed by $U$, is upstream. Indices $U$ and $D$ will also be used for various physical quantities in the $U$ and $D$ regions, respectively. Depending on the sign of $P-P_{*}$, there are two cases. In the first one, $P>P_{*}$, phase 1 is downstream, phase 2 is upstream, and $j_{n}<0$. In the second, $P<P_{*}$, phase 2 is downstream, phase 1 is upstream, and $j_{n}>0$. Therefore we can write the unperturbed normal vector $\mathbf{n}$ as $\left(\operatorname{sgn} j_{n}, 0,0\right)$. Other unperturbed values are the following: $c_{n}=0$; $v_{x} / u=$ const $\equiv j=\left|j_{n}\right|=g\left|P-P_{*}\right|$ (the mass flux across the front in the downstream direction); $v_{\text {in }}$ $=j u_{i}(P) \equiv v_{i} ; \mathbf{v}_{i t}=0$ (index $i$ stands for $D$ or $U$ ); $\mathscr{K}=0$; and $\mathbf{e}_{t}=\left(0, \operatorname{sgn} j_{n}, 0\right)$ (the unit tangential vector).

Let the function $F(x, y, z, t)=x-\zeta(y, z, t)=0$ describe the (weakly) perturbed position of the front. As the un- 
perturbed state is stationary and possesses translational and axial symmetries, the dependence of all perturbed physical quantities upon $(y, z, t)$ can be chosen proportional to $\exp \left(q t+i k_{y} y\right)$. Linearizing Eqs. (111), (112), and (114)-(116) around the moving front, one arrives at the following dispersion relation:

$$
\begin{aligned}
q^{2}+ & 2 q \frac{k\left|j_{n}\right| u_{1} u_{2}}{u_{1}+u_{2}}\left(1+\frac{f k}{\left|j_{n}\right|}\right)+k^{2} j_{n}^{2} \frac{u_{U}-u_{D}}{u_{U}+u_{D}} u_{D} u_{U} \\
& +2 f k^{3}\left|j_{n}\right| \frac{u_{D}^{2} u_{U}}{u_{U}+u_{D}}=0
\end{aligned}
$$

where $k \equiv\left|k_{y}\right|$. The real part of one of its roots is always negative, while the other root,

$$
\begin{aligned}
q= & -\frac{k j u_{1} u_{2}}{u_{1}+u_{2}}\left[1+\frac{f k}{j}\right. \\
& \left.-\sqrt{1+\frac{f^{2} k^{2}}{j^{2}}+\left(\frac{u_{2}}{u_{1}}-\frac{u_{2}}{u_{1}}\right) \operatorname{sgn}_{n}-2 \frac{f k}{j} \frac{u_{D}}{u_{U}}}\right],
\end{aligned}
$$

can have a positive real part, which corresponds to instability. Indeed, one can see that evaporation fronts [for which, always, $j_{n}=g\left(P_{*}-P\right)>0$ ] are unstable $(\operatorname{Re} q>0)$ if the perturbation wavelength is sufficiently large:

$$
0<k<k_{0}=\frac{1}{2} \frac{j_{n}}{f} \frac{u_{1}}{u_{D}} \frac{u_{2}-u_{1}}{u_{2}+u_{1}} .
$$

The maximum growth rate can be estimated as $q \sim u_{1,2} j_{n}^{2} / f \sim\left(P-P_{*}\right)^{2}$. It obviously goes to zero with the pressure mismatch. When $P>P_{*}$, one has $\operatorname{Re} q<0$, so that condensation fronts are always stable.

It is important to notice that this stability problem is quite similar to the hydrodynamic stability problem for a laminar flame propagation, which was investigated in the pioneering works by Darrieus, Landau, and Markstein (see Landau and Lifshitz, 1987; Zel'dovich et al., 1985; Pelcé, 1988; Liñán and Williams, 1993). These works predicted a similar instability for sufficiently long perturbation wavelengths (the so-called DarrieusLandau instability). At short wavelengths, the instability is suppressed by the Markstein effect, related to the perturbation-induced front curvature. The similarity between the evaporation front instability and the DarrieusLandau instability becomes clear when one notices that the unperturbed state in both cases consists in a relatively slow (subacoustic) heat and mass flow through a hydrodynamic discontinuity. It is essential that $f>0$, so that no analogs of the thermodiffusive flame instability (Zel'dovich et al., 1985; Liñán and Williams, 1993) or Mullins-Sekerka instability in solidification (Kurz and Fisher, 1992) appear.

Nonlinear effects of the Darrieus-Landau instability in the flame front dynamics ("wrinkled flames") were considered in a number of works, starting from the original work of Landau (1944). The instability tends to increase the front's area, which leads to the front acceleration. In the extreme case, the front surface can even become fractal (Liñán and Williams, 1993). Laboratory experiments with spherical flames clearly show the Darrieus-Landau instability and fractalization of the front (Gostintsev et al., 1988). Recently, fractalization has been reproduced numerically (Filyand et al., 1994; Blinnikov and Sasorov, 1996; Blinnikov et al., 1995). In addition, Blinnikov and Sasorov (1996) and Blinnikov et al. (1995) have related the fractal dimension of the flame front surface to the density contrast of the flame, $\gamma_{f}=1-u_{1} / u_{2}$.

Theoretical approaches of Filyand et al. (1994), Blinnikov et al. (1995), and Blinnikov and Sasorov (1996) are based on simplified model equations describing the nonlinear stage of the Darrieus-Landau instability in the approximation of a potential flow (Sivashinsky, 1977; Frankel, 1990). Strictly speaking, this approximation is valid only in the limit of a small density contrast, $\gamma_{f} \ll 1$. Formally applying it to the case of $\gamma_{f} \sim 1$, Filyand et al. (1994) obtained numerical results quite similar to the experimental results of Gostintsev et al. (1988). Of course, one cannot expect that the potential approximation will work well in the problem of the evaporation front instability in, say, interstellar medium, which is usually characterized by very large density contrasts. As large $\gamma_{f}$ lead to strong vorticity generation, it is quite possible that the evaporation fronts will be destroyed by turbulence.

The same transverse instability of the evaporation fronts can also develop in a confined plasma. As the pressure mismatch $P-P_{*}$ (and therefore the flux of material through the discontinuity $j_{n}$ ) is not constant in this case, the problem is more complicated. However, there is a simple limit of a "strong" instability, when (at least, the linear) theory remains valid. Indeed, the pressure mismatch $P-P_{*}$ can be regarded as almost constant, if the characteristic growth rate of the Darrieus-Landautype instability is much larger than the typical inverse time $\tau_{1}^{-1}$ of the pressure relaxation towards $P_{*}$. Assuming that neither $u_{1}$ nor $u_{2}$ introduce large or small parameters, we can write this inequality as $\left|P-P_{*}\right| \gg 1 /\langle L\rangle$, where $\langle L\rangle$ is the typical size of patterns. Correspondingly, an opposite inequality is a necessary criterion for the validity of the stability analyses of confined plasmas in all cases, where evaporation takes place.

Let us briefly summarize the results of Sec. VII.F. Condensation fronts are always stable with respect to a transverse (Darrieus-Landau-type) instability. Therefore, in isobaric plasmas, the (quasi)planar condensation fronts, developing after the radiative segregation stage is completed, will lead to the system uniformization, as predicted by the planar theory. In confined plasmas, the curvature-dominated condensation fronts' dynamics, reviewed in Secs. VII.C-VII.E are also unchallenged. On the contrary, the evaporation fronts' dynamics can be quite complicated (both in the isobaric case and at an earlier stage of the confined plasmas' dynamics). One can speculate, however, that the latest stage of the dynamics of radiative condensation patterns in a confined plasma will be always dominated by curvature, as the 
plasma pressure finally approaches $P_{*}$, and the growth rate of the Darrieus-Landau-type instability vanishes.

\section{RADIATIVE CONDENSATIONS AND MAGNETIC FIELDS}

In this section we shall briefly consider the role of magnetic fields in the radiative condensation dynamics. That this role can be crucial (and can strongly modify many of the effects described previously) becomes clear already in the linearized theory. The pioneering work by Field (1965) studied thermal instability of a plasma in a uniform magnetic field. It is obvious that for perturbations with wave vectors parallel to the field, the field does not affect the dynamics. If the wave vector is normal to the magnetic field, the field introduces two additional effects. The first is due to the magnetic pressure; the second (if the plasma is an ideal conductor) is the property of the field line to be frozen into the plasma. These two factors combined impede and anisotropize the plasma condensation. For perturbations oblique to the magnetic field, the problems become more complicated. In the simplest linearized theory, one obtains a dispersion relation of the fifth order (Field, 1965), as two new modes (Alfvén and magnetoacoustic waves) appear in addition to the three "old" modes. We shall deal in this section with the two simplest problems, which are related to the radiative condensation dynamics of magnetized plasmas. The first of them describes the radiative condensation dynamics in the idealized case when the condensations develop across a straight magnetic field with no shear. In Sec. VIII.A, we follow Meerson, Priest, and Steele (1993) and show that, mathematically, this problem can be reduced to the planar problem with no magnetic field that we considered before. Section VIII.B treats another, relatively simple case when the magnetic field is sufficiently strong, so that it completely suppresses the plasma motions across the field (but not the transverse thermal conduction). In this case, the plasma is able to redistribute itself along the field in response to the heat propagation across the field. This simplified model was developed by Meerson, Petviashvili, and Tajima (1995) in an attempt to better understand the basic physics of MARFEs in tokamak edge plasmas.

\section{A. Development of condensations across the magnetic field}

Considering one-dimensional motions of a fully ionized plasma across a magnetic field, we employ a slab model. The magnetic field $B(x, t)$ has straight field lines, and a plasma with the density $\rho(x, t)$ is allowed to move across the field (i.e., along the $x$ axis) with velocity $v(x, t)$ under the action of the gradient of the total (thermal + magnetic) pressure, $p(x, t)+B^{2}(x, t) / 8 \pi$. The governing magnetohydrodynamics equations are (see, e.g., Priest, 1984)

$\frac{d \rho}{d t}+\rho \frac{\partial v}{\partial x}=0$, $\rho \frac{d v}{d t}=-\frac{\partial}{\partial x}\left(p+\frac{B^{2}}{8 \pi}\right)+\eta \frac{\partial^{2} v}{\partial x^{2}}$,

$\frac{d}{d t}\left(\frac{B}{\rho}\right)=0$,

$\frac{1}{\gamma-1} \frac{d p}{d t}+\frac{\gamma}{\gamma-1} p \frac{\partial v}{\partial x}+\rho \mathscr{B}(\rho, T, B)-\frac{\partial}{\partial x}\left(K_{\perp} \frac{\partial T}{\partial x}\right)=0$,

$p=\frac{R}{\mu} \rho T$,

where the heat-loss function can now depend also on the magnetic field $B$.

The simplest, spatially uniform equilibria of these equations, $\rho_{0}, T_{0}, p_{0}$, and $B_{0}$, satisfying the condition $\mathscr{L}\left(\rho_{0}, T_{0}, B_{0}\right)=0$, are unstable with respect to the radiative condensation mode if

$$
T_{0} \mathscr{L}_{T}-\frac{\rho_{0} \mathscr{L}_{\rho}+B_{0} \mathscr{L}_{B}}{1+\gamma c_{A}^{2} / c_{s}^{2}}<0
$$

(cf. Field, 1965). Indices $T, \rho$, and $B$ denote derivatives with respect to the corresponding variable, evaluated at its equilibrium value. Furthermore, $c_{s}=\left(\gamma R T_{0} / \mu\right)^{1 / 2}$ is the velocity of sound, and $c_{A}=B_{0} /\left(4 \pi \rho_{0}\right)^{1 / 2}$ is the Alfvén velocity. Notice that the instability condition usually becomes more stringent in comparison to that for an unmagnetized plasma. Physically, it is natural, as the radiatively condensing plasma has to overcome the (building up) magnetic pressure.

Similar to the unmagnetized plasma case, reduction of the governing equations is possible if there are strong inequalities between the characteristic time scales (correspondingly, length scales) of the problem. The characteristic time scales of the problem are the radiative cooling time $\tau_{r}$, the magnetoacoustic time $\tau_{M A}$ $=\lambda /\left(c_{A}^{2}+c_{s}^{2}\right)^{1 / 2}$, and the transverse thermal conduction time $\tau_{T C}=\lambda^{2} / \kappa_{\perp}$, where $\lambda$ is a typical dimension of the initial perturbation and $\kappa_{\perp}=K_{\perp} / \rho_{0}$ is the transverse thermal diffusivity.

In the intermediate-wavelength limits, we have $\tau_{M A} \ll \tau_{r} \ll \tau_{T C}$, and it is the force equilibrium that sets in on the fastest, magnetoacoustic time scale. Then a slower stage of evolution starts when the density, temperature, velocity, and magnetic-field perturbations evolve on the background of a uniform total pressure:

$$
\frac{\partial}{\partial x}\left[p(x, t)+\frac{B^{2}(x, t)}{8 \pi}\right]=0 .
$$

In the long-wavelength limit, radiation is much faster than the magnetoacoustic motions. In this case thermal equilibrium

$$
\mathscr{L}(\rho, T, B)=0
$$

sets in first, and then slower magnetoacoustic motions start (we have neglected the transverse thermal conduction in the last condition). Meerson, Priest, and Steele (1993) considered both limits. The simplest case is when the system evolves from a small initial perturbation 
around an unstable uniform equilibrium. In this case the ratio $B / \rho$ remains constant in time [see Eq. (149)]; therefore, in both the intermediate- and longwavelength limits, one can replace Eq. (149) by the simple condition

$$
\frac{B(x, t)}{\rho(x, t)}=\frac{B_{0}}{\rho_{0}}=\text { const. }
$$

This relation enables one to eliminate the magnetic field from the governing equations. As a result, one obtains, in each of the limits, reduced sets of equations, which are very similar to the corresponding sets of equations derived for radiative condensation in unmagnetized plasmas with the heat-loss function, effective pressure, etc., modified. Meerson, Priest, and Steele (1993) studied the dynamics of developing radiative condensation in these two limits. We shall not present those results here, as they closely resemble those for unmagnetized plasmas after the (more stringent) instability criterion (152) has been satisfied. An important difference is obtained in the intermediate-wavelength limit, in the regime when the total pressure is constant. One finds that the maximum possible compression of the unstable plasma is

$$
\frac{\rho_{\max }}{\rho_{0}}=\left(1+\frac{8 \pi R \rho_{0} T_{0}}{\mu B^{2}}\right)^{1 / 2},
$$

which implies that $\beta_{0}=8 \pi R \rho_{0} T_{0} /\left(\mu B^{2}\right)$ must be much larger than 1 , if significant plasma condensations across the field are to be achieved (see also Karpen et al., 1989). Other details can be found in Meerson, Priest, and Steele (1993).

\section{B. Multifaceted asymmetric radiation from the edge of tokamak plasmas (MARFE): simple dynamic model}

Impurity radiation at the edge of a tokamak discharge is well known to represent one of the major factors determining the global stability of the discharge (Vershkov and Mirnov, 1974; Gibson, 1976; Murakami et al., 1976; Rebut and Green, 1977; Ohyabu, 1979). At the so-called density limit, the total impurity radiation power becomes equal to the total input power to the tokamak discharge. Above the density limit, the discharge undergoes a thermal collapse (Rebut and Green, 1977; Ohyabu, 1979; Ashby and Hughes, 1981). MARFEs are observed as standing or moving strongly radiating belts of short poloidal and radial extent at the edge of tokamak plasmas. They develop when the density reaches about $25 \%$ of the density limit. The plasma density in the MARFE can become comparable to that in the center of the discharge. Accordingly, the plasma temperature in the MARFE is strongly decreased. MARFEs were observed in almost every tokamak (Terry et al., 1981; Alladro et al., 1982; Baker et al., 1982; Lipshultz et al., 1984; Boody et al., 1985; Lipshultz, 1987; Sergienko et al., 1993). They significantly increase the impurity radiation power. On the other hand, experiments with reproducible MARFEs have been suggested as a means for edge-plasma diagnostics (Sergienko et al., 1993). By now it is clear that MARFEs represent radiative condensations (Stringer, 1985; Neuhauser et al., 1986).

Theoretical studies of MARFEs have proceeded in three directions: (i) the linear analysis of the radiative condensation instability, (ii) search for possible MARFE-like equilibria of a tokamak edge plasma, (iii) attempts to simulate the process of MARFE formation numerically. Drake (1987) was the first to understand that an adequate theory of MARFEs must be at least two dimensional (2D). Using fluid equations in cylindrical geometry, he analyzed the linear stability of a simple (constant density, but radially nonuniform temperature) thermal equilibrium. He showed that the radiative condensation instability starts when the plasma density exceeds a critical threshold that is somewhat lower than the density limit, in agreement with observations. Furthermore, he found that the poloidally symmetric perturbations are normally damped, which explains the observed poloidal asymmetry of MARFEs. Subsequent extensions of Drake's linear theory addressed ionization-recombination balance, edge-density gradients, different forms of the radiative cooling function and the "detached plasma" regime (Choudhury and Kaw, 1989; Morozov, 1992; Deshpande, 1994). It is important that the instability threshold has been found quite insensitive to the details of the (poorly known) radiative cooling function (Drake, 1987; Deshpande, 1994).

The search for possible MARFE-like equilibria involves the analysis of a two-dimensional thermal equilibrium equation, which includes a (nonlinear) heatingcooling function and anisotropic heat conduction. Krasheninnikov (1988) and Kaw et al. (1990) analyzed simplified versions of this equation for possible poloidally symmetric and asymmetric (MARFE-like) solutions and the transitions between them. Dependence of the transverse heat conduction on the poloidal angle (resulting from the corresponding magnetic-field dependence) was introduced by Bazdenkov et al. (1990), in an attempt to interpret the localization of steady-state MARFEs at the smaller major radius side of tokamaks. [An alternative to this mechanism is the toroidal variation on the plasma density which, because of an enhanced radiation, provides a "seed" for the radiative condensation instability at the smaller major radius side (Drake, 1987).]

The linear stability and 2D equilibrium analyses, though quite informative, are not sufficient for elucidating the physics of MARFE formation. This problem requires a nonlinear time-dependent analysis. Neuhauser et al. (1986) studied the nonlinear radiative condensation instability dynamics numerically, employing a onedimensional multifluid code with the ionizationrecombination balance for the hydrogen and impurities. A number of interesting effects were found, such as relaxation oscillations during a plasma density rise, condensation front propagation, and effects related to inhomogeneous power and impurity input. However, it 
became clear, after the works of Drake (1987), Krasheninnikov (1988), and Kaw et al. (1990), that at least the 2D nonlinear dynamics should be addressed. It has been done recently by Meerson, Petviashvili, and Tajima (1995).

Similar to a number of previous studies of MARFEs [see, e.g., Drake (1987)], Meerson, Petviashvili, and Tajima (1995) assumed that the tokamak magnetic field completely suppresses plasma motions across the field, but not the transverse heat conduction. They started with the simple fluid equations:

$$
\begin{aligned}
& \frac{d n}{d t}+n \vec{b} \cdot \vec{\nabla} v_{\|}=0, \\
& m_{i} n \frac{d v_{\|}}{d t}=-\vec{b} \cdot \vec{\nabla} p, \\
& \frac{3}{2} \frac{d p}{d t}+\frac{5}{2} p \vec{b} \cdot \vec{\nabla} v_{\|}-\vec{b} \cdot \vec{\nabla} \kappa_{\|} \vec{b} \cdot \vec{\nabla} T-\vec{\nabla}_{\perp} \cdot \kappa_{\perp} \vec{\nabla}_{\perp} T \\
& \quad+L-H=0,
\end{aligned}
$$

where $n, T$, and $p=n T$ are the plasma concentration, temperature, and pressure, respectively; $\vec{b}=\vec{B} / B$ is a unit vector along the magnetic field $\vec{B} ; v_{\|}$is the longitudinal (along $\vec{B}$ ) velocity; $m_{i}$ is the ion mass; $\kappa_{\|}$and $\kappa_{\perp}$ are the coefficients of longitudinal and transverse heat conduction; $L=n^{2} F(T)$ is the radiative cooling function; and $\mathrm{H}$ is a model heating function. The total time derivative in Eqs. (154)-(156) is $d / d t=\partial / \partial t+v_{\|} \vec{b} \cdot \vec{\nabla}$.

Proceeding in the spirit of reduced models of radiative condensations, Meerson, Petviashvili, and Tajima (1995) explicitly used the fact that the typical longitudinal acoustic time scale is normally much shorter than the other relevant time scales (the radiative cooling time scale and longitudinal and transverse heat-conduction time scales). In this combined intermediate- and shortwavelength limit, the plasma pressure rapidly becomes uniform along the magnetic-field lines, so that the complete Euler equation (155) can be replaced by the simple relation $\vec{b} \cdot \vec{\nabla} p=0$.

It is known that MARFEs develop in a thin region at the plasma edge. Therefore Meerson, Petviashvili, and Tajima (1995) considered the problem in slab geometry $(x, z)$, supplementing it by periodic boundary conditions with respect to the magnetic-field direction $z$. The left boundary of the plasma, $x=0$, was kept at a constant temperature $T=T_{0}$ (this condition simulated the hot plasma inside the discharge), while the right boundary, $x=a$, was kept at a (significantly lower) constant temperature $T_{a}$, which simulated the discharge periphery. The boundary conditions along $z$ were periodic: $n(x, 0)=n(x, l), T(x, 0)=T(x, l)$, and $v_{\|}(x, 0)=v_{\|}(x, l)$, where $l=2 \pi R$, and $R$ was identified with the major radius of the tokamak. From this periodicity immediately follows mass conservation along $z$ :

$$
M(x, t)=\int_{0}^{l} n(x, z, t) d z=\operatorname{const}(t) .
$$

Introduce the specific volume of the fluid, $u(x, z, t)=n^{-1}(x, z, t)$, and eliminate the temperature, using the equation of state. We arrive at the following two equations for the three variables $u(x, z, t)$, $v_{\|}(x, z, t)$, and $p(x, t)$ :

$$
\begin{aligned}
& \frac{d u}{d t}-u \frac{\partial v_{\|}}{\partial z}=0, \\
& \frac{3}{2} \frac{\partial p}{\partial t}+\frac{5}{2} p \frac{\partial v_{\|}}{\partial z}-p \frac{\partial}{\partial z}\left(\kappa_{\|} \frac{\partial u}{\partial z}\right)-\frac{\partial}{\partial x}\left(\kappa_{\perp} \frac{\partial(p u)}{\partial x}\right) \\
& \quad+L-H=0,
\end{aligned}
$$

where it is assumed that $\kappa_{\|}, \kappa_{\perp}, L$, and $H$ are expressed as functions of $u$ and $p$. Integrating Eq. (159) with respect to $z$ over the period $l$, we obtain a nonlocal evolution equation for the pressure:

$$
\frac{\partial p}{\partial t}=-\frac{2}{3 l} \int_{0}^{l}(L-H) d z+\frac{2}{3 l} \int_{0}^{l} \frac{\partial}{\partial x}\left(\kappa_{\perp} \frac{\partial(p u)}{\partial x}\right) d z .
$$

This equation represents one more version of the global pressure balance equation. Equations (158)-(160) form a closed set and represent the Eulerian version of the reduced model of Meerson, Petviashvili, and Tajima (1995). They can be further simplified by introducing a Lagrangian mass coordinate along the $z$ axis:

$$
m(x, z, t)=\int_{0}^{z} u^{-1}\left(x, z^{\prime}, t\right) d z^{\prime} .
$$

Transforming to the coordinates $x$ and $m$, we are left with only two governing equations:

$$
\begin{aligned}
\frac{\partial u}{\partial t}= & -\frac{2 u}{5 p}(L-H)-\frac{3 u}{5 p} \frac{\partial p}{\partial t}+\frac{2}{5 M^{2}(x)} \frac{\partial}{\partial s}\left(\frac{\kappa_{\|}}{u} \frac{\partial u}{\partial s}\right) \\
& +\frac{2 u}{5 p}\left(\frac{\partial}{\partial x}+\Upsilon \frac{\partial}{\partial s}\right) \kappa_{\perp}\left[\frac{\partial}{\partial x}(p u)+\Upsilon \frac{\partial}{\partial s}(p u)\right], \\
\frac{\partial p}{\partial t}= & \frac{2 M(x)}{3 l} \int_{0}^{1} u\left(\frac{\partial}{\partial x} k+\Upsilon \frac{\partial}{\partial s}\right) \kappa_{\perp}\left[\frac{\partial}{\partial x}(p u)+\Upsilon \frac{\partial}{\partial s}(p u)\right] d s \\
& -\frac{2 M(x)}{3 l} \int_{0}^{1} u(L-H) d s,
\end{aligned}
$$

where $M(x)=\int_{0}^{l} u^{-1}(x, z, t) d z$ is the ( $x$ dependent) total mass content of each magnetic-field line. Because of mass conservation along $z$, the quantity $M(x)$ is independent of time and determined solely by the initial density profile. As usual, the constancy of the system length $l$ in the $z$ direction, while trivial in the Eulerian coordinates, appears as a conservation law in the Lagrangian description:

$$
\int_{0}^{M(x)} u(x, m, t) d m=l .
$$

Furthermore, $s=s(x, z, t)=m(x, z, t) / M(x)$, while $\mathrm{Y}=\mathrm{Y}(x, z, t)=\partial s / \partial x$ is assumed to be expressed through $s, x$, and $t$.

Equation (162) for $u(x, m, t)$ should be solved subject to the periodic boundary condition with respect to $m$, 

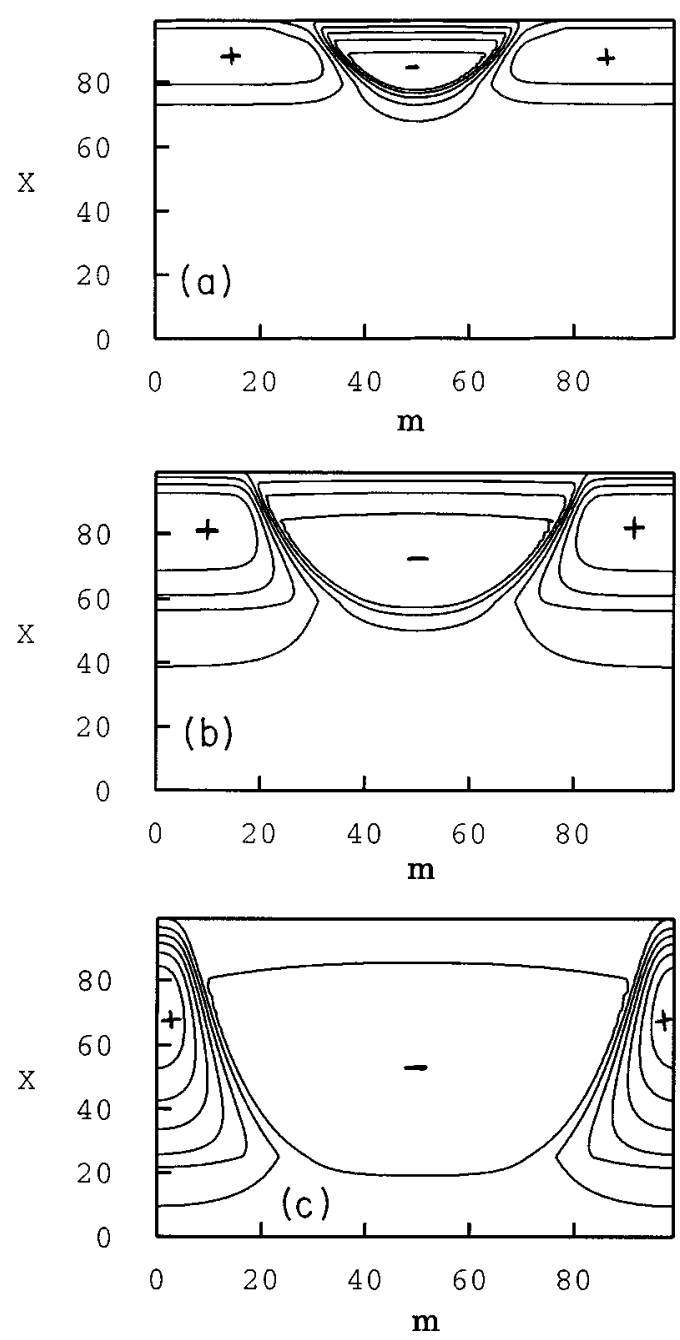

FIG. 21. Formation of a MARFE-like two-dimensional pattern. Shown are equidistant contours of the specific volume of the plasma $u$ as a function of the coordinate $x$ and Lagrangian coordinate $m$ at successive times $t=18$ (a), 36 (b), and 90 (c). + and - indicate the maximum $u_{+}$and minimum $u_{-}$values of $u$, where $u_{+}=1.37, u_{-}=0.13(\mathrm{a}), u_{+}=1.96, u_{-}=0.083(\mathrm{~b})$, and $u_{+}=4.28, u_{-}=0.074$ (c). By the time $t=90$, a steady state is apparently achieved. The system lengths in the $x$ and $z$ directions are 15 and 120, respectively.

$u[x, m=M(x), t]=u(x, m=0, t)$. The equation is coupled to the nonlocal evolution equation (163). Again, this nonlocality is related to some global constraints [this time, of a constant mass content $M(x)$ and length $l$ of the system along the magnetic field]. We have already discussed similar nonlocalities in thermally bistable plasmas, in one, two, and three dimensions. The nonlocalities introduce "global negative feedback" and contribute to the formation of persistent stationary structures, normally impossible in nonconstrained one-component reaction-diffusion-type equations. As we have seen, the mechanism of stationary pattern formation in bistable systems with global negative feedback consists, essentially, in the arrest of the motion of "phase-transition" fronts, which otherwise would finally make the system

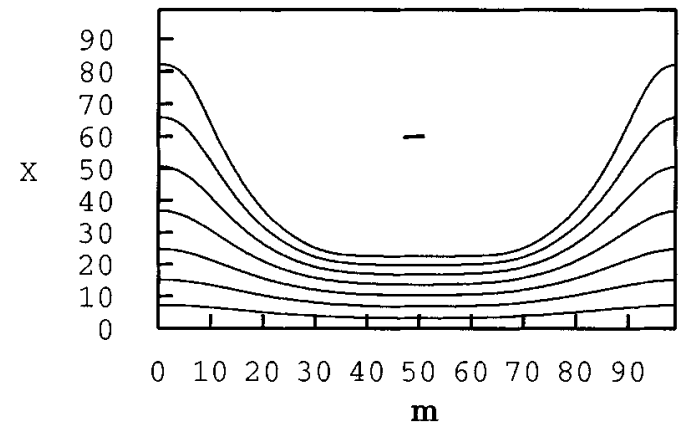

FIG. 22. Formation of a MARFE-like two-dimensional pattern. Shown is the plasma temperature (equidistant contours) as a function of coordinate $x$ and Lagrangian coordinate $m$ at time $t=90$. The system lengths in the $x$ and $z$ directions are 15 and 120 , respectively. "- " indicates the minimum temperature $T_{-}=0.68$ (a flat plateau). The maximum temperature $T_{0}=45$ is kept constant at $x=0$, and $T=T_{a}=1$ at $x=15$.

uniform. The arguments, developed for the bistable systems, can be extended to the more complicated (in particular, nonbistable) 2D model (162), (163), especially in the case when a one-dimensional (1D) constant-density equilibrium, similar to that considered by Drake (1987), the cylindrical geometry, is unstable with respect to the radiative condensation instability. Therefore Meerson, Petviashvili, and Tajima (1995) interpreted the development of MARFEs in terms of the formation of stationary patterns (in the form of poloidally asymmetric segregated states) in a more complicated reaction-diffusiontype system with global negative feedback.

To verify this concept and follow the MARFE formation, Meerson, Petviashvili, and Tajima (1995) solved the reduced equations (162) and (163) numerically. As neither the linear theory of the radiative condensation instability (Drake, 1987; Deshpande, 1994) nor the MARFE-like equilibria (Krasheninnikov, 1988; Kaw et al., 1990) are very sensitive to the precise form of the cooling function, Meerson, Petviashvili, and Tajima (1995) followed Drake (1987) to adopt a rather crude model of the line radiation from the tokamak edge: $L=u^{-2} L_{0}$, for the temperature interval $T_{a} \leqslant p u \leqslant T_{L}$, and $L=0$ outside this interval. In addition, $T_{L}$ was taken to be much smaller than $T_{0}$, so that the radiative cooling was localized near the edge. For simplicity, they assumed the following model forms of the heat conduction coefficients: $\kappa_{\|}=T=p u, \kappa_{\perp}=1$. (Renormalizing properly the coordinates $x$ and $m$ and rescaling the dimensions $a$ and $l$, we can always scale down the numerical coefficients in the heat conduction coefficients.) The simplest case of a zero heating, $H=0$, was chosen.

Figures 21 and 22 show a typical example of the model edge-plasma dynamics in the case when a MARFE-like condensation develops. The scaled edgeplasma dimensions were $a=15$ and $l=120$, while the parameters were the following: $T_{0}=45, T_{a}=1, T_{L}=3$, and $L_{0}=3$. In this run, the authors started with a small 2D seed plasma condensation with a maximum density perturbation of $5 \times 10^{-2}$ on the background of a constant (and equal to 1) plasma density. The seed conden- 
sation was exponentially localized close to the middle of the "cold wall" of the system. For this initial density profile, the mass content of each magnetic-field line was almost independent of $x$ and equal to $M=120$ (with an accuracy better than $10^{-3}$ ). Recall that $M$ serves as the Lagrangian "length" of the system in the $z$ direction. The initial pressure profile was one dimensional and linear in $x: p(x, m, t=0)=T_{0}-\left(T_{0}-T_{a}\right)(x / a)$. The constancy of $l$ [Eq. (164)] was checked in order to monitor the accuracy of computations.

As the initial conditions are not in thermal equilibrium, even without the small perturbation (they would be if it were not for the radiative cooling), the system starts to evolve. The region with temperature less than $T_{L}$ is cooling down, as the radiative cooling operates here, while the heat conduction supplies heat from the core plasma and tends to smooth the temperature profile. Simultaneously, however, the initial small perturbation grows, and a pronounced $2 \mathrm{D}$ (poloidally asymmetric) condensation develops. Figures 21(a) and 21(b) for the specific-volume dynamics in the coordinates $x$ and $m$ clearly show that the condensation results from plasma inflow along the magnetic field. Finally, an apparently stationary, localized, poloidally asymmetric condensation is formed, as seen in Fig. 21(c) for the specific volume and in Fig. 22 for the temperature. (Remember that $m$ is the Lagrangian mass coordinate. In the original Eulerian coordinate $z$, the poloidal extent of the growing condensation is a few times smaller.)

As expected, MARFE-like condensations develop in this model if the radiation intensity $L_{0}$ exceeds a threshold value $L_{0 *}$. With all of the other parameters unchanged, $L_{0 *}$ is found to be about 2.1. (As the authors did not start from any unstable 1D equilibrium, this threshold was not expected to coincide with that predicted by any linear theory.) Below the threshold, a onedimensional (poloidally symmetric) equilibriumtemperature profile sets in, and no condensation develops. Above the threshold, the poloidal and radial extent of the steady-state condensation grows with the "supercriticality" $L_{0}-L_{0 *}$ (it is essential that it remains nonzero when $L_{0}-L_{0 *}>0$ goes to zero). Finally, simulations with other initial pressure profiles give similar results; therefore Meerson, Petviashvili, and Tajima (1995) conclude that the MARFE-like structures have an extensive basin of attraction in the space of initial conditions.

An immediate extension of this simple model would be taking into account dependence of the transverse heat conduction on the coordinate $z$. This dependence can simulate the dependence on the poloidal angle, resulting from the corresponding magnetic-field variation. This addition will enable us to verify the mechanism of localization of steady-state MARFEs at the smaller major radius side of tokamaks suggested by Bazdenkov et al. (1990). Another extension of the model would include an attempt to simulate the phenomenon of detached plasma and its role in the development of MARFEs. [This effect was investigated by Morozov (1992) in the linear theory.] To this end, one should in- troduce a low-temperature cutoff in the radiative loss function at a temperature somewhat higher than the "cold" boundary temperature $T_{a}$.

\section{CONCLUDING REMARKS}

We have reviewed recent progress in studying the nonlinear dynamics of radiative condensations in optically thin plasmas. This progress has been made possible due to an explicit use of the hierarchy of characteristic time scales (and corresponding length scales) in the system. A number of reduced models have been developed in recent years, which address the dynamics of radiative condensation in the long-, intermediate-, and shortwavelength limits. Sometimes these models have analogs in other physical and chemical problems, which facilitates the "cross-fertilization" between different fields. Being much simpler than the original unreduced gasdynamic (or magnetogasdynamics) equations, the reduced models prove to be very helpful in understanding the basic physics of radiative condensation formation and development. Sometimes, useful analytic solutions describing the dynamics of radiative condensation can be obtained.

As the radiative condensation instability is usually strong, in the sense that the final state of the system is very different from the initial one, the overall behavior of the heating-cooling function in a wide range of temperatures and densities becomes important. Some useful insight into the nature of radiative condensation can be obtained when this function is approximated in such a way that the radiative condensation instability proceeds "unlimitedly." We have observed that conventional (advective) nonlinearities are usually unable to stop the unstable growth, and radiative condensations (or, alternatively, radiative rarefactions) in such cases look like explosions (that is, develop singularities in a finite time).

A more realistic picture is obtained in the regime of bistability. In this case, the process of radiative condensation leads naturally to segregation of the radiatively cooling gas into two "phases" with quite different properties: a cool and dense phase coexisting (at least, temporarily) with a hot and dilute phase. This process takes a time of the order of the characteristic radiative cooling time of the system in the intermediate-wavelength limit, and of the order of the acoustic time in the longwavelength limit. (We give these estimates for the idealized case of an isotropic plasma and assume that the temperatures of the cool and hot regions do not introduce large or small parameters.) The further dynamics prove to be determined by the character of the boundary conditions, namely, whether the system is "open" or "closed."

In open systems (plasma inflow or outflow is allowed), one of the locally stable phases is actually metastable and is finally "consumed," so that radiative segregation proves to be a transient phenomenon with a lifetime proportional to the average size of a single pattern of each of the phases. The process of relaxation towards a uniform equilibrium proceeds via temperature (and den- 
sity) fronts of evaporation or condensation. The nature of these fronts is very different in the intermediate- and long-wavelength limits. In the intermediate-wavelength limit, the fronts are similar to those predicted by Zel'dovich and Pikel'ner (1969) and by Penston and Brown (1970). It has been found recently that, in the case of evaporation, the fronts are unstable with respect to transverse perturbations, and this instability (which is very similar to the well-known Darrieus-Landau instability of laminar flame propagation) can lead to front fractalization, acceleration, and, possibly, turbulence. In the long-wavelength limit, the fronts represent shock waves of a new type, in which the gas density profile is monotonic, but the gas pressure profile is not. These shocks prove to be stable with respect to small longitudinal and transverse perturbations.

In closed or confined systems, the physics of radiative condensations can be very different. The presence of a nonlocal, integral constraint (mass conservation) imposed on the dynamics makes it possible to arrest the front motion and thus to facilitate a much longer (even "eternal") coexistence of the two phases. The characteristic time scale of the relaxation of the confined system to this coexistence state is proportional to the average size of a single domain of each of the phases. It is interesting that these general results are independent of the time-scale hierarchy used in the theory, as they are obtained both in the intermediate- and in the longwavelength limits.

Many features of the planar dynamics of radiative condensations in confined plasmas persist in a more complicated situation, where the plasma motion remains one dimensional (along a strong magnetic field), while the (anisotropic) thermal conduction acts both along and across the magnetic field. Such a model seems to be the simplest possible one that provides a physically reasonable description of the formation of MARFEs in tokamak edge plasmas. Although no complete theory has been developed for this model, numerical results with this model show that, with sufficient radiative cooling, the nonlocal constraint (mass conservation) does lead to formation of steady-state MARFE-like structures. Further work in this direction will possibly enable us to interpret more of the observed properties of MARFEs.

The previous example shows that reduced models of radiative condensations can be useful in more complicated situations, when other forces, in addition to pressure gradient, are present. Another such example represents the recently considered problem of "quasihydrostatic" unsteady flows of radiatively cooling selfgravitating gas clouds (Meerson, Megged, and Tajima, 1996). In this problem, it is the approximate balance between the pressure gradient and self-gravity force (and, possibly, Ampère force) that sets in quickly. Then a "slow" flow develops which can lead to very significant effects (like radiative collapse or explosion) on a relatively long, radiative time scale.

In general, the dynamics of nonplanar radiative condensations in a confined plasma can be quite different from those of the planar ones. Indeed, we have seen that later-stage dynamics are governed (in the intermediatewavelength limit) by the curvature of the interfaces between the two phases. The front-curvature effects become dominant when the radiative segregation has already been established, the plasma pressure is close to the area-rule value $P_{*}$, and the interface motion slows down. Then, on a longer time scale, which is proportional to the square of the size of a typical pattern, the curvature effects lead to smoothening of such patterns. Possible types of large-scale equilibrium patterns are determined, in confined systems, by the condition of a constant mean curvature of their interfaces, showing similarity to the classical problem of the equilibrium of weightless capillary surfaces. Therefore the simplest individual equilibrium clouds or voids in such systems are perfect balls, two and three dimensional. The stability of these objects with respect to various types of perturbations has been analyzed. Small perturbations of the perfect shape of the equilibrium patterns are shown to be damped out. On the other hand, the instability of a single spherical cloud (which we call a drop), with respect to purely radial perturbations, has been described, and the minimum radius of a stable equilibrium drop has been determined. Smaller drops either shrink and disappear, or expand until they reach a new, stable equilibrium. Furthermore, background-mediated competition (Ostwald ripening) in an ensemble of drops has been predicted. Larger drops always thrive at the expense of smaller drops, and only the largest drop can finally survive. There exists a one-parameter family of "universal" self-similar distribution functions of the (many) clouds with respect to their sizes. Correspondingly, there are power-law scalings of the pressure mismatch, cloud density, and average cloud radius with time. Solving the selfsimilar problem alone does not provide any selection rule for this family of solutions, so that additional arguments (employing a certain feature of the initial condition) must be invoked. The (selected) self-similar solution represents an intermediate asymptotics of the dynamics, as finally only the largest cloud survives. As can be expected, many of these phenomena have similarities with those occurring during first-order phase transitions.

Nonplanar dynamics of radiative condensations in the long-wavelength regime also represents an interesting problem. In 1987, the prediction was made about "thermal pancakes" developing during the late stage of radiative collapse. This prediction was based on the similarity between the late stage of radiative collapse and the late stage of well-studied gravitational collapse, where the celebrated "Zel'dovich's pancakes" are thought to develop. A more realistic approach to this problem must take into account the eventual stabilization of the radiative condensation instability at large densities. While the corresponding theory has been elaborated for planar geometry, its three-dimensional extensions are still lacking. We expect that flattening will remain intact in the more complete theory. However, many details are still unclear; in particular, the role of "normal-anomalous" planar shock waves. As these shock waves have been shown to 
be stable with respect to three-dimensional perturbations, they can play an important role in the late stage of radiative collapse.

The original motivation behind the studies of the radiative condensation instability and related segregation processes in optically thin plasmas was to explain the strong inhomogeneities observed in many astrophysical plasmas (such as interstellar and intergalactic clouds and voids, and solar prominences) and, more recently, radiative condensations in laboratory plasmas (such as MARFEs). It has been found that a variety of stable, strongly segregated equilibria is indeed possible and accessible in confined plasmas (in contrast to isobaric plasmas, where the final state is normally uniform). The simple "perfect" objects that we have found to be in stable equilibrium seem to be good candidates for "regular" radiative condensation structures observed in astrophysics and in the laboratory (like "simple" interstellar clouds, solar prominences, and MARFEs). On the contrary, they cannot explain the frequently observed complexity in the geometric shapes of many interstellar clouds and voids. One can assume that some of the observed complexity might be related to earlier, more violent stages of the dynamics, long before any equilibrium was reached. Indeed, the evaporation-fronts instability can lead to much more complicated (fractal) shapes of the evaporating clouds, so that further investigation of the nonlinear stage of this instability is necessary.

Obviously, more complicated patterns might also appear, if we take into account some of the effects that we neglected (gravity, rotation, complicated magnetic-field geometry, ionization-recombination dynamics, etc.). Now that substantial progress in the study of the basic paradigms of radiative segregation has been achieved, these questions are worth addressing.

\section{ACKNOWLEDGMENTS}

I have benefited very much from collaboration on radiative condensations with I. Aranson, A.M. Dimits, A.M. Milne, N. Petviashvili, E.R. Priest, C.D.C. Steele, and T. Tajima. I also wish to acknowledge the advice of J.F. Drake, M. Marder, H. R. Strauss, R.A.M. Van der Linden, and A. Vilenkin. I am especially grateful to my old friend P.V. Sasorov for long-time collaboration and many enjoyable discussions, in Moscow and Jerusalem, during the last 20 years. This work was supported in part by the Israel Science Foundation.

\section{REFERENCES}

Akhromeyeva, T.S., S.P. Kurdyumov, G.G. Malinetskii, and A.A. Samarsky, 1989, Phys. Rep. 176, 189.

Alfimov, G.L., V.M. Eleonsky, N.E. Kulagin, L.M. Lerman, and V.P. Silin, 1990, Physica D 44, 168.

Alladro, F., R. Bartiromo, B. Casali, P. Buratti, and F. DeMarco, 1982, Phys. Lett. A 90, 405.

Antiochos, S.K., and A.J. Klimchuk, 1991, Astrophys. J. 378, 372.
Aranson, I., B. Meerson, and P.V. Sasorov, 1993, Phys. Rev. E 47, 4337.

Aranson, I., B. Meerson, and P.V. Sasorov, 1995, Phys. Rev. E 52, 948.

Arnold, V. I., Ya. B. Zel'dovich, and S. F. Shandazin, 1981, Preprint No. 100, Institute for Applied Mathematics, Academy of Sciences of the U.S.S.R., Moscow.

Ashby, D.E.T.F., and M.H. Hughes, 1981, Nucl. Fusion 21, 911.

Baker, D.R., R.T. Snider, and N. Nagami, 1982, Nucl. Fusion 22, 807.

Balbus, S.A., 1988, Astrophys. J. 328, 395.

Barelko, V.V., V.M. Beřbutyan, Yu.E. Volodin, and Ya.B. Zel'dovich, 1981, Dokl. Akad. Nauk SSSR 257, 339 [Sov. Phys. Dokl. 26, 335 (1981)].

Barenblatt, G.I., 1979, Similarity, Self-Similarity and Intermediate Asymptotics (Consultants Bureau, New York).

Batchelor, G.K., 1970, An Introduction to Fluid Dynamics (Cambridge University, Cambridge, England), p. 244.

Bazdenkov, S.V., A.V. Gruzinov, and O.P. Pogutse, 1990, Plasma Phys. Controlled Fusion 32, 1061.

Begelman, M.C., and C.F. McKee, 1990, Astrophys. J. 358, 375. Belova, N.G., A.A. Galeev, R.Z. Sagdeev, and Yu.S. Sigov, 1980, Pis'ma Zh. Éksp. Teor. Fiz. 31, 551 [JETP Lett. 31, 518 (1980)].

Blinnikov, S.I., and P.V. Sasorov, 1996, Phys. Rev. E (in press). Blinnikov, S.I., P.V. Sasorov, and S.E. Woosley, 1995, "Selfacceleration of nuclear flames in supernovae," in Proceedings of the Jubilee Gamov Seminar, St. Petersburg, Russia, September 1994, edited by A. Bykov, Space Sci. Rev. (in press).

Bogoyavlenskii, O.I., 1985, Methods in the Qualitative Theory of Dynamical Systems in Astrophysics and Gas Dynamics (Springer, Berlin).

Boody, F.P., C.E. Bush, S.S. Medley, H. Park, and J. Schivell, 1985, Bull. Am. Phys. Soc, 30, 1518.

Braginskii, S.I., 1957, Zh. Éksp. Teor. Fiz. 33, 645 [Sov. Phys. JETP 6, 494 (1958)].

Brown, L.C., 1989, Acta Metall. 37, 71.

Brown, L.C., 1990, Scr. Metall. Mater. 24, 963, 2231.

Brown, L.C., 1992, Acta Metall. Mater. 40, 1293.

Burton, W.B., B.G. Elmegreen, and R. Genzel, 1992, Eds., The Galactic Interstellar Medium (Springer, Berlin).

Chaplygin, S.A., 1976, Izbrannye Trudy, Selected Works (Nauka, Moscow), p. 94 (in Russian).

Chen, M.K., and P.W. Voorhees, 1993, Model. Simul. Mater. Sci. Eng. 1, 591.

Chiuderi, C., and G. Van Hoven, 1979, Astrophys. J. 232, L69.

Choe, G.S., and L.C. Lee, 1992, Sol. Phys. 138, 291.

Choudhury, S.R., and P.K. Kaw, 1989, Phys. Fluids B 1, 1646.

Cross, M.C., and P.C. Hohenberg, 1993, Rev. Mod. Phys. 65, 851.

Dalgarno, A., and R.A. McCray, 1972, Annu. Rev. Astron. Astrophys. 10, 375.

Defouw, R.J., 1970a, Astrophys. J. 160, 659.

Defouw, R.J., 1970b, Astrophys. J. 161, 55.

Deshpande, S., 1994, Phys. Plasmas 1, 127.

Dimits, A.M., and B. Meerson, 1991, Phys. Fluids B 3, 1420.

Doroshkevich, A.G., and Ya.B. Zel'dovich, 1981, Zh. Éksp.

Teor. Fiz. 80, 801 [Sov. Phys. JETP 53, 405 (1981)].

Drake, J.F., 1987, Phys. Fluids 30, 2429.

Drake, J.F., Y. Mok, and G. Van Hoven, 1993, Astrophys. J. 413, 416.

Drake, J.F., L. Sparks, and G. Van Hoven, 1988, Phys. Fluids 31, 813. 
Dyakov, S.P., 1954, Zh. Éksp. Teor. Fiz. 27, 288.

Elmer, F.J., 1992, Z. Phys. B 87, 377.

Elphick, C., O. Regev, and N. Shaviv, 1992, Astrophys. J. 392, 106.

Engvold, O., and E. Jensen, 1977, Sol. Phys. 52, 37.

Ferrara, A., and Yu. Shchekinov, 1993, Astrophys. J. 417, 595.

Field, G.B., 1965, Astrophys. J. 142, 531.

Field, G.B., D.W. Goldsmith, and H.J. Habing, 1969, Astrophys. J. Lett. 155, L149.

Fife, P., 1979, Mathematical Aspects of Reacting and Diffusing Systems, Lecture Notes in Biomathematics, Vol. 28 (Springer, New York).

Filyand, L., G.I. Sivashinsky, and M.L. Frankel, 1994, Physica D 72, 110.

Finn, R., 1986, Equilibrium Capillary Surfaces (Springer, New York), p. 133.

Fisher, P., 1936, Ann. Eugenics 7, 355.

Frankel, M. L., 1990, Phys. Fluids A 2, 1879.

Frank-Kamenetskii, D.A., 1969, Diffusion and Heat Transfer in

Chemical Kinetics (Plenum, New York), p. 99.

Gibson, A., 1976, Nucl. Fusion 16, 546.

Goldsmith, D.W., 1970, Astrophys. J. 161, 41.

Gostintsev, Ya.A., A.G. Istratov, and Yu.V. Shulenin, 1988, Combust. Explos. Shock Waves 24, 70.

Gray, A., 1993, Modern Differential Geometry of Curves and Surfaces (CRC, Boca Raton), p. 280.

Gurevich, A.Vl., and R.G. Mints, 1987, Rev. Mod. Phys. 59, 941.

Haken, H., 1978, Synergetics. An Introduction, Springer Series in Synergetics Vol. 1 (Springer, Berlin/Heidelberg).

Haken, H., 1987, Advanced Synergetics, Springer Series in Synergetics Vol. 20 (Springer, Berlin/Heidelberg).

Heyvaerts, J., 1974, Astron. Astrophys. 37, 65.

Hildner, E., 1974, Sol. Phys. 35, 123.

Hollenbach, D.J., and H.A. Thronson, Jr., 1987, Eds., Interstellar Processes, Astrophysics and Space Science Library, Vol. 134 (Reidel, Dordrecht).

Kadomtsev, B.B., 1963, in Voprosy Teorii Plazmy, edited by M.A. Leontovich (Atomizdat, Moscow), Vol. 2, p. 173 [Rev. Plasma Phys. 2, 195 (1966)].

Kaplan, S.A., and S.B. Pikel'ner, 1979, Physics of the Interstellar Medium (Nauka, Moscow; in Russian).

Kaptsov, O.V., 1988, Dokl. Akad. Nauk SSSR 298, 597 [Sov. Phys. Dokl. 32, 44 (1988)].

Karpen, J.T., S.K. Antiochos, J.M. Picone, and R.B. Dahlburg, 1989, Astrophys. J. 338, 493.

Karpen, J.T., J.M. Picone, and R.B. Dahlburg, 1988, Astrophys. J. 324, 590.

Kaw, P.K., S. Deshpande, K. Avinash, and S. Rath, 1990, Phys. Rev. Lett. 65, 2873.

Kittel, C., and H. Kroemer, 1980, Thermal Physics (Freeman, San Francisco), p. 290.

Klimchuk, J.A., S.K. Antiochos, and J.T. Mariska, 1987, Astrophys. J. 320, 409.

Kolmogorov, A.N., I.G. Petrovskii, and N.S. Piskunov, 1937, Byull. Mosk. Gos. Univ., Sekt. Matematika i Mekhanika 1, No. 6, p. 1 [Bull. Univ. Moscow, Ser. Internat., Sec. A 1, 1 (1937)].

Krasheninnikov, S.I., 1988, Pis'ma Zh. Éksp. Teor. Fiz. 48, 287

[JETP Lett. 48, 320 (1988)].

Kurz, W., and D.J. Fisher, 1992, Fundamentals of Solidification

(Trans Tech, Aedermannsdorf, Switzerland).
Lamb, H., 1932, Hydrodynamics (Cambridge University Press, Cambridge).

Landau, L.D., 1944, Acta Physicochim. URSS 19, 77 (see also a reprinted version in Pelcé, 1988).

Landau, L.D., and E.M. Lifshitz, 1987, Fluid Mechanics (Pergamon, Oxford).

Lawson, J.D., 1959, J. Nucl. Energy, Part C 1, 31.

Lepp, S., R. McCray, J.M. Shull, D.T. Woods, and T. Kallman, 1985, Astrophys. J. 288, 58.

Lifshitz, I.M., and V.V. Slezov, 1958, Zh. Éksp. Teor. Fiz. 35, 479 [Sov. Phys. JETP 8, 331 (1959)].

Liñán, A., and F.A. Williams, 1993, Fundamental Aspects of Combustion (Oxford University Press, New York).

Lipshultz, B., 1987, J. Nucl. Mater. 145-147, 15.

Lipshultz, B., B. LaBombard, E.S. Marmar, M.M. Pickrell, J.L. Terry, R. Watterson, and S.M. Wolfe, 1984, Nucl. Fusion 24, 977.

Manneville, P., 1990, Dissipative Structures and Weak Turbulence, Perspectives in Physics, edited by H. Araki, A. Libchaber, and G. Parisi (Academic, Boston).

Marder, M., 1987, Phys. Rev. A 36, 858.

McCarthy, D., and J.F. Drake, 1991, Phys. Fluids B 3, 22.

Meerson, B., 1989, Astrophys. J. 347, 1012.

Meerson, B., E. Megged, and T. Tajima, 1996, Astrophys. J. 457, 321.

Meerson, B., N. Petviashvili, and T. Tajima, 1995, Phys. Plasmas 2, 414.

Meerson, B., E.R. Priest, and C.D.C. Steele, 1993, Geophys. Astrophys. Fluid Dyn., 71, 243.

Meerson, B., and I.M. Rutkevich, 1994, unpublished.

Meerson, B.I., and P.V. Sasorov, 1987, Zh. Éksp. Teor. Fiz. 92, 531 [Sov. Phys. JETP 65, 300 (1987)].

Meerson, B., and P.V. Sasorov, 1996, Phys. Rev. E (in press).

Meerson, B., C.D.C. Steele, A.M. Milne, and E.R. Priest, 1993, Phys. Fluids B 5, 3417.

Middya, U., M. Sheintuch, M.D. Graham, and D. Luss, 1993, Physica D 63, 393.

Mikhailov, A.S., 1990, Foundations of Synergetics I. Distributed Active Systems, Springer Series in Synergetics Vol. 51 (Springer, Berlin).

Mok, Y., J.F. Drake, D.D. Schnack, and G. Van Hoven, 1990, Astrophys. J. 359, 228.

Morozov, D.Kh., 1992, Fiz. Plazmy 18, 564 [Sov. J. Plasma Phys. 18, 294 (1992)].

Murakami, M., J.D. Callen, and L.A. Berry, 1976, Nucl. Fusion 16, 347.

Murray, J.D., 1989, Mathematical Biology (Springer, Berlin).

Nedospasov, A.V., and V.D. Khait, 1979, Oscillations and Instabilities in Low-Temperature Plasmas (Nauka, Moscow; in Russian).

Neudachin, V.V., and P.V. Sasorov, 1991, Nucl. Fusion 31, 1053.

Neuhauser, J., S. Schneider, and R. Wunderlich, 1986, Nucl. Fusion 26, 1679.

Ohyabu, N., 1979, Nucl. Fusion 19, 1491.

Oran, E.S., J.T. Mariska, and J.P. Boris, 1982, Astrophys. J. 254, 349.

Ostwald, W., 1900, Z. Phys. Chem. 34, 495.

Parker, E.N., 1953, Astrophys. J. 117, 431.

Pease, R.S., 1957, Proc. R. Soc. London Ser. B 70, 11.

Pelcé, P., 1988, Dynamics of Curved Fronts (Academic, Boston).

Penston, M.V., and F.E. Brown, 1970, Mon. Not. R. Astron. Soc. 150, 373. 
Pikel'ner, S.B., 1967, Astron. Zh. 44, 915 [Sov. Astron. 11, 737 (1968)].

Pikel'ner, S.B., 1971, Sol. Phys. 17, 44.

Pismen, L., 1979, Chem. Eng. Sci. 34, 563.

Poland, A.I., and J.Y. Mariska, 1986, Sol. Phys. 104, 303.

Priest, E.R., and E.A. Smith, 1979, Sol. Phys. 64, 267.

Priest, E.R., 1984, Solar Magnetohydrodynamics (Reidel, Dordrecht), p. 91.

Priest, E.R., 1989, Ed., Dynamics and Structure of Quiescent Solar Prominences, Astrophysics and Space Science Library, Vol. 150 (Kluwer, Dordrecht).

Raju, P.K., 1968, Mon. Not. R. Astron. Soc. 139, 479.

Rebut, P.H., and B.J. Green, 1977, Nucl. Fusion., Suppl., 2, 3.

Ribes, E., and W. Unno, 1980, Astron. Astrophys. 91, 129.

Rosner, R., W.H. Tucker, and G.S. Vaiana, 1978, Astrophys. J. 220, 643.

Rubinstein, J., and P. Sternberg, 1992, IMA J. Appl. Math. 48, 249.

Ruždjak, V., and E. Tandberg-Hanssen, 1989, Eds., Dynamics of Quiescent Prominences (Springer, Berlin).

Sasorov, P.V., 1975, Astron. Zh. 52, 106 [Sov. Astron. 19, 62 (1975)].

Sasorov, P.V., 1988, Pis'ma Astron. Zh. 14, 306 [Sov. Astron. Lett. 14, 129 (1988)].

Scheffler, H., and H. Elsässer, 1987, Physics of the Galaxy and Interstellar Matter (Springer, Berlin).

Schwarz, J., R. McCray, and R.F. Stein, 1972, Astrophys. J. 175, 673.

Sergienko, G., K. Höthker, A. Nedospasov, A. Pospieszczyk, D. Rusbüldt, U. Samm, B. Schweer, and M. Tokar, 1993, in 20th European Conference on Controlled Fusion and Plasma Physics, Contributed Papers, Part II, Lisboa, edited by J.A. Costa Cabral, M.E. Manso, F.M. Serra, and F.C. Schüller (European Physical Society, Lisboa), p. 667.

Shercliff, J.A., 1977, J. Fluid Mech. 82, 687.
Sivashinsky, G.I., 1977, Acta Astronautica 4, 1177.

Slezov, V. V., 1978, J. Phys. Chem. Solids 39, 367.

Slezov, V.V., and V.V. Sagalovich, 1987, Usp. Fiz. Nauk 151, 67 [Sov. Phys. Usp. 30, 23 (1987)].

Sparks, L., G. Van Hoven, and D.D. Schnack, 1990, Astrophys. J. 353, 297.

Spitzer, L., 1978, Physical Processes in the Interstellar Medium (Wiley, New York), p. 2.

Stringer, T.E., 1985, in 12th European Conference on Controlled Fusion and Plasma Physics, Budapest, Hungary, 1985, edited by L. Pocs and A. Montvai (European Physical Society, Budapest), Part 1, p. 86.

Stuart, J.T., 1967, J. Fluid Mech. 29, 417.

Tandberg-Hanssen, E., 1974, Solar Prominences (Reidel, Dordrecht).

Tenorio-Tagle, G., M. Moles, and J. Melnik, 1989, Eds., Structure and Dynamics of the Interstellar Medium, Lecture Notes in Physics Vol. 350 (Springer, Berlin).

Terry, J.L., E.S. Marmar, and S.M. Wolfe, 1981, Bull. Am. Phys. Soc. 26, 886.

Trubnikov, B.A., and S.K. Zhdanov, 1987, Phys. Rep. 155, 137. Van der Linden, R.A.M., M. Goossens, and J.P. Goedbloed, 1991, Phys. Fluids B 3, 866.

Vershkov, V.A., and S.V. Mirnov, 1974, Nucl. Fusion 14, 383.

Wagner, C., 1961, Z. Elektrochem. 65, 581.

Yao, J.H., K.R. Elder, H. Guo, and M. Grant, 1993, Phys. Rev. B 47, 14110 .

Zel'dovich, Ya.B., G.I. Barenblatt, V.B. Librovich, and G.M. Makhviladze, 1985, The Mathematical Theory of Combustion and Explosions (Consultants Bureau, New York).

Zel'dovich, Ya.B., and I.D. Novikov, 1983, The Structure and Evolution of the Universe, Relativistic Astrophysics Vol. 2 (University of Chicago, Chicago).

Zel'dovich, Ya.B., and S.B. Pikel'ner, 1969, Zh. Éksp. Teor. Fiz. 56, 310 [Sov. Phys. JETP 29, 170 (1969)]. 